\title{
Abstracts of the 13th German Allergy Congress
}

\author{
Dresden, September 27-29, 2018
}

Online publiziert: 30 August 2018

(C) Springer Medizin Verlag GmbH, a part of Springer Nature 2018

\section{Epidemiology and environment}

\section{P1.1}

\section{Spättypsensibilisierungen bei der Nahrungsmittelherstellung}

\section{A. Bauer}

Klinik und Poliklinik für Dermatologie, Universitätsklinikum Carl Gustav Carus, TU Dresden, Dresden, Deutschland

Die Berufskrankheit nach Nr. 5101 steht mit einem Anteil von über $40 \%$ an erster Stelle aller gemeldeten Berufskrankheiten in Nahrungsmittelberufen. Daten des Nordbayerischen Berufsdermatosenregisters zeigen, dass $12 \%$ der Bäcker, $10 \%$ der Konditoren und $5 \%$ der im Register erfassten Köche unter allergischen Kontaktekzemen litten. Der Vortrag gibt einen Überblick über Hauptallergene und Sensibilisierungshäufigkeiten sowie Sensibilisierungsrisiko in verschiedenen Berufen der Nahrungsmittelindustrie. Das relative Risiko, ein berufsbedingtes Handekzem in der Lebensmittelverarbeitung zu entwickeln, ist mehr als 3-fach erhöht. Vor allem betroffen sind Bäcker, Konditoren, Köche und Fleisch- und Fischverarbeiter. Neben irritativen Kontaktekzemen treten häufig allergische Kontaktekzeme und Proteinkontaktdermatitis auf. Auslösende Allergene sind Gummichemikalien, aber auch Wirkstoffe in Desinfektionsmitteln und pflanzliche Allergene.

Wenige Allergene sind in der Nahrungsmittelherstellung für die überwiegende Mehrzahl der berufsrelevanten Typ IV Sensibilisierungen verantwortlich.

\section{P1.2}

\section{Relation between birch pollen count and combined symptom and medication score in birch allergic patients}

\section{U. Berger ${ }^{1}$, T. Higenbottam ${ }^{2}$, D. Lee ${ }^{3}$, D. Wessiepe ${ }^{4}$, S. Rückinger ${ }^{4}$, M. Skinner ${ }^{2}$}

${ }^{1}$ Research Group Aerobiology and Pollen Information, Department of Otorhinolaryngology, Vienna, Austria ${ }^{2}$ Allergy Therapeutics, Worthing, United Kingdom ${ }^{3}$ Bencard Allergie GmbH, Munich, Germany ${ }^{4}$ Metronomia Clinical Research, Munich, Germany

Introduction: The Combined Symptom and Medication Score (CSMS) has been recommended by EAACI as primary endpoint for pivotal Phase 3 studies in allergic rhinoconjunctivitis (ARC). A Phase III study with a modified birch allergen subcutaneous immunotherapy (SCIT) product with modified allergen tyrosine adsorbate (MATA) and monophosphoryl lipid A (MPL) will read-out after the birch pollen season of 2018. To optimize the analysis plan using the CSMS, the birch pollen data of the European Aeroallergen Network (EAN) was used to identify the window within the birch pollen season (BPS) with optimal correlation between the birch pollen counts (BPC) and the CSMS.

Methods: EAN currently includes information from $>400$ active and 300 historical pollen-monitoring stations in Europe (39 countries). The EAN database used for analysis included birch pollen data from 2009-2016. The daily symptoms and medication were recorded using an APP questionnaire. The CSMS was re-calculated using the symptom and medication scores. The relation between the daily BPC and the daily CSMS was analyzed.

Results: Days of the BPS with higher BPC had significantly higher CSMS values. Irrespective of BPC, for earlier days during BPS statistically significant higher CSMS values were observed. Furthermore, during the overlap of the BPS and the grass pollen season (GPS) lower CSMS values were reported.

Conclusions: These results confirm a statistically significant correlation between BPC and CSMS. Importantly, these 
findings suggest that the optimal window to observe treatment effects may be a short interval after start of the BPS and during the peak BPS, due to generally higher CSMS values. This provides sufficient basis to consider additional sensitivity analyses to evaluate the treatment effect of birch MATA MPL SCIT on the primary CSMS endpoint during a shortened window after the start of the BPS and to consider excluding the overlapping period between the BPS and GPS from the primary analysis.

\section{P1.3}

\section{Relation between grass pollen count and combined symptom and medication score in grass allergic patients}

\section{U. Berger ${ }^{1}$, T. Higgenbottam ${ }^{2}$, D. Lee ${ }^{3}$, D. Wessiepe ${ }^{4}$, S. Rückinger ${ }^{4}$, M. Skinner $^{2}$}

${ }^{1}$ Research Group Aerobiology and Pollen Information, Department of Otorhinolaryngology, Vienna, Austria

${ }^{2}$ Allergy Therapeutics, Worthing, United Kingdom

${ }^{3}$ Bencard Allergie $\mathrm{GmbH}$, Munich, Germany

${ }^{4}$ Metronomia Clinical Research, Munich, Germany

Introduction: The Combined Symptom and Medication Score (CSMS) has been recommended by EAACI as primary endpoint for pivotal Phase III studies in allergic rhinoconjunctivitis (ARC). A Phase II study with a modified grass allergen subcutaneous immunotherapy (SCIT) product with modified allergen tyrosine adsorbate (MATA) and monophosphoryl lipid A (MPL) adjuvants will read out in 2018. To optimize the analysis plan using the CSMS, the grass pollen data of the European Aeroallergen Network (EAN) was used to identify the window within the grass pollen season (GPS) with optimal correlation, between the grass pollen counts (GPC) and CSMS.

Methods: EAN currently includes information from $>400$ active and 300 historical pollen-monitoring stations in Europe (39 countries). The EAN database used for analysis included grass pollen data from 2009-2016. The daily symptoms and medication were recorded using an App questionnaire. The CSMS was re-calculated using the symptom and medication scores and the correlation of CSMS to the daily GPC was analyzed.

Results: Days of the GPS with higher grass pollen counts had significantly higher CSMS values. Irrespective of the GPC, for earlier days during the GPS statistically significant higher CSMS values were observed. Furthermore, during the overlap of the GPS and the birch pollen season (BPS) lower CSMS values were reported.

Conclusions: These results confirm a statistically significant correlation between GPC and the CSMS. Importantly, these findings suggest that the optimal window to observe treatment effects may be a short interval after start of the GPS and during the peak GPS, due to generally higher CSMS values. This provides sufficient basis to consider additional sensitivity analyses to evaluate the treatment effect of grass MATA MPL SCIT on the primary CSMS endpoint during a shortened window after the start of the GPS and to consider excluding the overlapping period between the BPS and GPS from the primary analysis.

\section{P1.4}

\section{Sensibilisierungen gegenüber Aeroallergenen im Raum Göttingen im Zeitverlauf zwischen 1998-2017}

\section{Beutner', S. Forkel', T. Fuchs', M. Schön'1, J. Geier², T. Buhl'}

${ }^{1}$ Klinik für Dermatologie, Venerologie und Allergologie, Universitätsmedizin Göttingen, Göttingen, Deutschland ${ }^{2}$ Informationsverbund dermatologischer Kliniken (IVDK), Göttingen, Deutschland

Zielsetzung: Sensibilisierungsraten gegenüber Aeroallergenen zeigen weltweit eine steigende Tendenz. Um Sensibilisierungsraten dominierender Aeroallergene einer Teilbevölkerung Deutschlands zu beschreiben, wurde diese Untersuchung durchgeführt.

Methoden: Retrospektiv ausgewertet wurden anonymisierte Hautpricktest-Ergebnisse symptomatischer Patienten der Klinik für Dermatologie, Venerologie und Allergologie Göttingen 1998-2017. Verglichen wurden sämtliche Testpatienten aus Vierjahresintervallen 1998-2001 (2093 Patienten), 2006-2009 (1034 Patienten) und 2014-2017 (1188 Patienten) mit annähernd konstantem Geschlechterverhältnis, hinsichtlich Sensibilisierungen gegenüber den drei wichtigsten Aeroallergengruppen frühblühende Baumpollen (Birke, Erle, Hasel), Gräser- und Roggenpollen sowie Hausstaubmilben (D. pteronyssinus [D. $p$.] und $D$. farinae $[D . f]$.$) .$

Ergebnisse: Insgesamt zeigen sich hohe Sensibilisierungsraten in unserem selektionierten, zunehmend älter werdenden Patientenkollektiv (Patientenalter $>40$ Jahre: $43,7 \%$ vs. $52,4 \%$ vs. $54,6 \%$ ). Es zeichnet sich eine deutliche Zunahme der Sensibilisierungsraten gegen alle Aeroallergene mit Ausnahme der Roggen- und Erlenpollen im zeitlichen Verlauf ab. Die hohen Sensibilisierungsraten für Gräser, Roggen, Birke und Erle dominieren alle drei Intervalle. Die initial deutlich geringeren Sensibilisierungen gegen $D$. p. und $D$.f. gleichen sich diesen im Verlauf an. Kreuzreaktionen innerhalb der Allergengruppen zwischen Birke, Erle und Hasel (76,6\% vs. $73,6 \%$ vs. $84,7 \%)$, sowie zwischen D. p. und D.f. (73,3 \% vs. $77,8 \%$ vs. $83,2 \%)$ steigen zum dritten Intervall (2014-17) hin an. Kreuzreaktionen zwischen Gräser und Roggen blieben weitgehend konstant $(89,9$ vs. 88,0 vs. $86,5 \%)$.

Schlussfolgerung: Auch in den Pricktest-Daten einer großen regionalen Patientenkohorte zeigen sich über die vergangenen 20 Jahre eine Zunahme von positiven Pricktest-Ergebnissen sowie Verschiebungen im Sensibilisierungsprofil gegenüber Aeroallergenen. 


\section{P1.5}

\section{„Selbst aktiv bei Allergie“ - Evidenz- und webbasiert Patientenkompetenz bei leicht bis mittelschweren allergischen Erkrankungen des atopischen Formenkreises erhöhen}

\section{E.-M. Bitzer ${ }^{1}$, K. Drixler ${ }^{1}$, J. Lander ${ }^{2}$, J. Hansert ${ }^{3}$, E. Luntz ${ }^{4}$, I. Schäfer ${ }^{5}$, J. Schmitt ${ }^{4}$, M. Dierks ${ }^{2}$}

${ }^{1}$ Pädagogische Hochschule Freiburg, Freiburg, Deutschland ${ }^{2}$ Medizinische Hochschule Hannover, Hannover, Deutschland ${ }^{3}$ Technik und Wirtschaft, Hochschule Karlsruhe, Karlsruhe, Deutschland

${ }^{4}$ Universitätsklinikum Dresden, Dresden, Deutschland ${ }^{5}$ Universitätsklinikum Hamburg Eppendorf, Hamburg, Deutschland

Hintergrund: Evidenz- und webbasierte Informationsund Lernangebote versprechen Unterstützung für Menschen mit leichten bis mittelschweren Allergien. Ziel dieser Studie ist es, Such- und Informationsanlässe, favorisierte Inhalte, sowie präferierte didaktische Formate aus der Nutzerperspektive zu ermitteln und diese Erkenntnisse bei der Entwicklung von „Selbst aktiv bei Allergie“ zu berücksichtigen.

Methode: In vier Fokusgruppen mit $(n=33)$ Betroffenen wurde die Nutzerperspektive eruiert und inhaltsanalytisch ausgewertet. Die Ergebnisse bildeten zusammen mit der aktuell verfügbaren Evidenz aus Leitlinien und qualitativ hochwertigen Übersichtsarbeiten sowie gesundheitspädagogischen und mediendidaktischen Überlegungen die Grundlage für die Entwicklung.

Ergebnisse: Betroffene suchen dann nach Informationen, wenn sie einen konkreten Handlungsbedarf haben. Von einer webbasierten Intervention erwarten sie: a) Individualisierungsmöglichkeiten, b) flexible Lernumgebung, c) Vermittlung von Handlungs- und Entscheidungsstrategien, e) Methodenvielfalt sowie f) interaktive Elemente.

Dementsprechend beinhaltet „Selbst aktiv bei Allergie“ u. a. Lernmodule zu Behandlungsmöglichkeiten, Karenzmaßnahmen, Selbstmanagement, sowie einen Arbeitsbereich für die Nutzer. Die Lerninhalte werden entlang symptomspezifischer Lernpfade und durch interaktive, zur Verhaltensänderung (z. B. Handlungsplanung) anregende Formate vermittelt.

Zusammenfassung: "Selbst aktiv bei Allergie“ ist ein niedrigschwelliges evidenz- und webbasiertes Informations- und Lernangebot für Menschen mit leichten bis mittelschweren Allergien des atopischen Formenkreises und als solches auf Symptome und Handlungsbedarf ausgerichtet. Es wird formativ optimiert und anschließend anhand von patientenrelevanten Zielkriterien kontrolliert evaluiert.

\section{P1.6}

\section{Zunahme der Kräuterpollen-Sensibilisierung im südlichen Niedersachsen über die vergangenen 20 Jahre}

\section{S. Forkel ${ }^{1}$, J. Geier ${ }^{2}$, C. Beutner ${ }^{1}$, A. Heetfeld ${ }^{1}$, T. Fuchs', M. Schön'1, T. Buhl'}

${ }^{1}$ Klinik für Dermatologie, Allergologie und Venerologie, Universitätsmedizin Göttingen, Göttingen, Deutschland ${ }^{2}$ Informationsverbund dermatologischer Kliniken (IVDK), Göttingen, Deutschland

Einleitung: Allergische Atemwegserkrankungen gehören zu den Erkrankungen mit einer sehr hohen Prävalenz und damit großer gesundheitlicher und sozioökonomischer Relevanz. Das Robert-Koch-Institut hat in der „Studie zur Gesundheit Erwachsener in Deutschland“ (DEGS 1, 2008-2011) eine Gesamtsensibilisierungsrate gegen Aeroallergene von 33,6\% und eine Sensibilisierungsrate von $11.2 \%$ gegen Beifuß- und Ambrosiapollen in der Normalbevölkerung festgestellt (jeweils Bestimmung von spezifischem IgE).

Methoden: In der retrospektiven Auswertung wurden Pricktestungen der Aeroallergene von insgesamt 6220 Patienten in der Universitätshautklinik Göttingen ausgewertet. Die Gesamtpopulation wurde in zwei Zeitdekaden aufgeteilt, (A) 1998-2007 und (B) 2008-2017. Die Untersuchung fokussierte auf die Sensibilisierungen von Wegerich, Ambrosia, Beifuß, Kamille, Brennnessel und Raps. Ebenfalls wurden die Co-Sensibilisierungen gegenüber Birke und Lieschgras analysiert.

Ergebnisse: Es zeigt sich eine Zunahme der Sensibilisierungsrate in der Dekade B (2008-2017) im Vergleich zu Dekade A (1998-2007) aller untersuchten Kräuterpollen, insbesondere von Wegerich und Ambrosia. Auffällig ist dabei eine deutliche Zunahme der Sensibilisierungen bei jüngeren Patienten (0-40 Jahre). Nur ein geringer Teil der Patienten (1998-2017: 24,9\%) zeigte eine Monosensibilisierung auf Kräuterpollen. Die Co-Sensibilisierung von Wegerich und Birkenpollen verdoppelte sich nahezu über das Zeitintervall (relativer Anstieg um 87,8\% auf $41,7 \%$ von allen Patienten). Zusammenfassung: Die mittels Pricktestung erhobene Sensibilisierungsrate im südlichen Niedersachsen gegen Kräuterpollen ist vergleichsweise hoch in Deutschland. Passend zu den vorhandenen Vorberichten zeigt sich auch in unseren Daten eine deutliche Zunahme der Sensibilisierungen in den vergangenen 20 Jahren.

\section{P1.7}

Aktuell, wissenschaftlich fundiert und unabhängig - der Allergieinformationsdienst am Helmholtz Zentrum München

\section{U. Koller'1, V. Braun', D. Burkhardt', F. Draeger'1, C. Peter ${ }^{1}$, H. Brosius' ${ }^{2}$, S. Opitz'}

${ }^{1}$ Abteilung Kommunikation, Allergieinformationsdienst, Helmholtz Zentrum München, Neuherberg, Deutschland ${ }^{2}$ Institut für Kommunikationswissenschaft und Medienforschung, Ludwig-Maximilians-Universität München, München, Deutschland

Hintergrund: Mit zunehmenden Erkrankungszahlen und zugleich steigenden Versorgungsengpässen sowie einer wach- 
senden Zahl von Patienten mit Selbstmedikation steigt der Bedarf an fundierter Information zu Allergien. In der Arztpraxis kann dieser angesichts knapper Zeitbudgets nicht immer umfassend gedeckt werden. So dient das Internet heute als Hauptinformationsquelle, doch vielen Angeboten fehlt die nötige Qualität und Neutralität. Prävention, frühe Diagnose und gezielte Behandlung von Allergien sind jedoch nur möglich, wenn Betroffene hinreichend informiert sind.

Zielsetzung: Ziel des seit 2017 am Helmholtz Zentrum München aufgebauten Allergieinformationsdienstes ist es, $\mathrm{Pa}$ tienten und anderweitig Interessierten direkt aus der Forschung wissenschaftlich fundierte Informationen zu Allergien bereitzustellen. Das Hauptaugenmerk liegt auf evidenzbasierter, unabhängiger und verständlich aufbereiteter Information. Das Bundesministerium für Gesundheit fördert den Aufbau.

Methoden: Im ersten Jahr wurde das online-Portal aufgebaut mit Basiswissen zu allergischen Krankheitsbildern, Diagnose- und Therapiemöglichkeiten sowie neuen Forschungsansätzen. Aktuelle Nachrichten aus der Forschung, Downloadangebote und Erklärvideos werden über Newsletter, Facebook und Twitter verbreitet. Eine Plattform informiert erstmals im deutschsprachigen Raum - über klinische Studien zu Allergien. Der Allergieinformationsdienst lädt auch zu Patiententagen ein. Ein wissenschaftlicher Beirat mit Vertretern führender Forschungseinrichtungen und Kliniken sowie der Fachgesellschaften unterstützt die hohe Qualität des Angebots.

Ergebnisse: Die meisten Inhalte des Portals (www.allergieinformationsdienst.de) werden in den Suchmaschinen bereits oben gelistet. Viele Patientenanfragen sowie Abdrucke in Fachund Publikumsmedien bestätigen den Bedarf an qualitätsgesicherter Information. Eine Evaluierungsstudie begleitet das Projekt, Ergebnisse aus den ersten Befragungen werden vorgestellt.

\section{P1.8}

\section{Atopische Beschwerden und Inanspruchnahme entsprechender medizinischer Versorgungs- leistungen bei erwachsenen Migranten in einer schwedischen Kohorte um 2010}

\section{J.C. Richter}

Abteilung für Arbeits- und Umweltmedizin, Universität Lund, Lund, Schweden

Einleitung: Allergische Erkrankungen stellen eine globale Herausforderung dar. Forscher, Kliniker und weitere Stakeholder aus Sozialwissenschaften, Stadtplanung und Gesundheitspolitik dürften ein Interesse daran haben, wie Umwelt und Lebensbedingungen im häuslichen Milieu den allergischen Phänotyp beeinflussen. Am Beispiel von internationalen Migranten können einzelne Faktoren eines komplexen Systems näher untersucht werden. Schweden ist seit Jahrzehnten ein klassisches Einwanderungsland. Migranten und Einwanderer aus verschiedenen Weltgegenden leben aus verschiedensten Gründen nicht selten konzentriert in Hochhaussiedlungen ausserhalb der Stadtzentren. Im Stadtteil Rosengård in Malmö wurde um 2008 festgestellt, dass eine erhebliche Anzahl von Wohnungen schwer verfallen und wegen Feuchtigkeitsschäden, Schimmelbefall und nicht funktionierender Lüftungen praktisch nicht mehr bewohnbar waren. Die Abteilung für Arbeitsund Umweltmedizin der Universität Lund plante die Durchführung einer Studie, um die Gesundheit dieser Familien vor und nach der Renovierung im sozialen Kontext darzustellen.
Methodik: Einschluss von Familien mit Kindern, die aufgrund symptomatischer respiratorischer Erkrankungen Kontakt mit dem Gesundheitssystem hatten (absichtliches Oversampling). Es wurden Hausbesuche organisiert, bei denen Paare von der jeweiligen Muttersprache der Teilnehmer mächtigen "Gesundheitskommunikatören“ die Familien besuchten: technische Inspektion der Wohnräume; Gesundheitsfragebogen für alle Mitglieder. Nach den Hausbesuchen Einladung zu diagnostischen Sitzungen mit dermalen Allergietests: Standardpanel luftgebundener Allergene. Analyse der offiziellen schwedischen Gesundheitsstatistiken.

Ergebnisse: 650 Teilnehmer aus 130 Haushalten, davon 359 Kinder. Hier vorgestellt werden Daten zu den Erwachsenen: selbst berichtete atopische Erkrankungen; Hauttests; Inanspruchnahme von Leistungen des Gesundheitssystems mit relevanten Diagnosen.

\section{P1.9}

\section{Die moderierende Rolle der Allergie Immuntherapie in der Progression von Asthma bronchiale}

\section{J. Schmitt', F. Tesch ${ }^{1}$, D. Küster ${ }^{1}$, N. Serup-Hansen ${ }^{2}$, V. Mücke ${ }^{2}$, L. Mocke ${ }^{1}$, E. Wüstenberg ${ }^{2}$}

'Zentrum für Evidenzbasierte Gesundheitsversorgung, Medizinische Fakultät Carl Gustav Carus, TU Dresden, Dresden, Deutschland

${ }^{2}$ ALK-Abelló, Hamburg, Deutschland

Hintergrund: Die Allergie-Immuntherapie (AIT) ist die einzige kausale Behandlungsmethode allergischer Atemwegserkrankungen. Präventive Langzeiteffekte sind für die allergische Rhinitis bei einigen Produkten dokumentiert. Bislang unklar ist, ob AIT in der Lage ist, die Progression von bereits bestehendem Asthma bronchiale zu verzögern oder zu verhindern.

Methode: Hierzu wurde eine Kohortenstudie durchgeführt, die auf Daten einer Krankenkasse basiert, welche Informationen über 1,74 Mio. Menschen aus Deutschland über einen Zeitraum von 10 Jahren beinhaltet. Personen mit Asthma wurden definiert als zwei ambulante ICD-10-Codes J45 (Asthma) und zwei Verschreibungen von Asthmamedikamenten. Die verordneten Asthmamedikamente wurden nach Behandlungsstufen gemäß dem von GINA 2006 empfohlenen Stufenschema gruppiert. Die Analyse erfolgte stratifiziert für die Altersgruppen 12-17 Jahre, 18-50 Jahre und >50 Jahre bezogen auf den Beginn des Beobachtungszeitraums (2005). Die Übergänge zwischen den GINA-Stufen wurden mittels Cox-Regressionsmodellen adjustiert für Alter und Geschlecht analysiert.

Ergebnisse: Medikamente der GINA Stufe 2 wurden kaum verordnet, so dass Übergänge zwischen GINA-Stufen 1, 3 und 4 analysiert werden konnten. Patienten mit AIT hatten ein reduziertes Risiko der Asthmaprogression von GINA Stufe 1 auf GINA Stufe 3 Hazard ratio (95\% CI): 0,87 (0,80-0,95). Dieser Effekt war bei jüngeren Patienten am stärksten ausgeprägt HR $0,72(0,58-0,88)$. Patienten mit AIT hatten auch ein verringertes Risiko einer Eskalation der Asthmatherapie von GINA Stufe 3 zu GINA Stufe 4 mit HR 0,66 $(0,60-0,74)$. Die Number needed to treat (NNT), um zu verhindern, dass ein Patient innerhalb von 5 Jahren von GINA 3 auf GINA 4 wechselt, betrug 10,9 $(8,2-16,2)$.

Schlussfolgerung: Diese routinedaten-basierte Kohortenstudie gibt wichtige Hinweise, dass eine AIT bei Patienten mit manifestem Asthma bronchiale das Fortschreiten der Erkrankung verhindern oder verzögern kann. 


\section{P1.10}

\section{Komorbiditäten von allergischer Rhinitis und Asthma}

\section{F. Tesch ${ }^{1}$, J. Schmitt ${ }^{1}$, D. Küster ${ }^{1}$, A. Domdey², E. Wüstenberg ${ }^{2}$}

'Zentrum für Evidenzbasierte Gesundheitsversorgung, Medizinische Fakultät Carl Gustav Carus, TU Dresden, Dresden, Deutschland

${ }^{2}$ ALK-Abelló, Hamburg, Deutschland

Hintergrund: Allergische Rhinitis (AR) und Asthma bronchiale (AA) sind eng verwandte Erkrankungen. Es ist bekannt, dass AR der Hauptrisikofaktor für die Entwicklung von AA ist. Ziel der vorliegenden Studie war es, Assoziationen von AR bzw. AA mit anderen Komorbiditäten zu untersuchen.

Methode: Es wurden Routinedaten einer gesetzlichen Krankenkasse verwendet und die Versicherten in vier Altersgruppen (A: 0-11 Jahre; B: 12-17 Jahre; C: 18-50 Jahre; D: 50+ Jahre) aufgeteilt. Die 100 häufigsten ICD-10-Diagnosen im Jahr 2014 wurden je Gruppe ausgewählt. Die Diagnosen wurden in einem Netzwerkdiagramm für jede Geschlechts-/Alterskombination ausgewertet. Die Stärke der Assoziation zwischen verschiedenen Diagnosen wurde durch Cramers V Korrelation berechnet.

Ergebnisse: Die Gesamtzahl der Komorbiditäten nahm mit den Altersgruppen zu. In der jüngsten Altersgruppe (A) waren sowohl AR als auch AA nur stark mit atopischen Erkrankungen wie atopischer Dermatitis korreliert. Bei Jugendlichen (Altersgruppe B) kamen Komorbiditäten wie Depressionen, somatoforme Störungen und Angstzustände hinzu, die besonders mit AA assoziiert sind. Bei Erwachsenen (Altersgruppe C) wurden die Assoziationen zu Depressionen, somatoformen Störungen und Angstdiagnosen noch stärker. Starke Korrelationen zur Muskel-Skelett-Diagnose wurden ebenfalls festgestellt, während die Assoziationen zu atopischen Erkrankungen weniger zum Tragen kamen. Viele Zusammenhänge wurden durch multimorbide Patienten, insbesondere in der Altersgruppe D, verstärkt.

Schlussfolgerung: Neben den bekannten Assoziationen von AR und AA mit anderen atopischen Erkrankungen wurde eine deutliche Korrelation von AR und AA mit psychischen Störungen dokumentiert. Die Kosten dieser Krankheiten sollten deshalb bei der Kostenberechnung für AR und AA berücksichtigt werden. Diese Analyse hilft zu verstehen, welche Komorbiditäten Lebensqualität und Kosten von Patienten mit AA und AR in verschiedenen Altersgruppen beeinflussen.

\section{P1.11}

\section{Atopie- und Asthmamarker bei jungen "gesunden und nicht allergischen" Personen}

\section{V. van Kampen, A. Deckert, O. Hagemeyer, M. Raulf, E. Marek, C. Monsé, T. Brüning, J. Bünger, R. Merget}

Institut für Prävention und Arbeitsmedizin der Deutschen Gesetzlichen Unfallversicherung, Institut der Ruhr-Universität Bochum (IPA), Bochum, Deutschland

Für inhalative Expositionsstudien wurden per Anzeige nichtallergische, nicht-rauchende gesunde Personen zwischen 18-55 Jahren gesucht. Die Eignung von interessierten Freiwil- ligen wurde zunächst mittels Fragebogen und anschließend durch eine Voruntersuchung überprüft, die u.a. ein Arztgespräch, eine körperliche Untersuchung, Sputuminduktion mit $0,9 \% \mathrm{NaCl}$, Blutabnahme, Messung von fraktioniertem exhalierten Stickstoffmonoxid (FeNO) sowie Lungenfunktions- und Methacholintest umfasste.

Insgesamt 86 Personen (54\% Frauen, Median: 25 (19-48) Jahre), die angegeben hatten nicht an einer Atemwegsallergie zu leiden, nahmen an der Voruntersuchung teil. Die gemessenen IgE-Werte auf Umweltallergene (sxl) lagen im Median bei $0,26 \mathrm{kU} / \mathrm{L}(0,05-93,7 \mathrm{kU} / \mathrm{L})$ und korrelierten signifikant mit dem Gesamt-IgE (35 kU/L (2-1348 kU/L), $p<0,0001)$, den FeNO-Werten (13 ppb (5-94 ppb), $p=0,036)$ sowie dem Anteil an Sputum-Eosinophilen (0\% (0-10\%), $p=0,005)$, nicht jedoch mit dem Anteil an Blut-Eosinophilen $(p=0,214) .41$ Personen ( $48 \%$ ) hatten sxl-Werte $\geq 0,35 \mathrm{kU} / \mathrm{L}$ und wurden als Atopiker eingestuft. Im Vergleich zu den 45 Nicht-Atopikern hatten diese ein signifikant höheres Gesamt-IgE $(p<0,0001)$ und einen höheren Anteil an Sputum-Eosinophilen ( $p=0,0045)$, während sich die FeNO-Werte der beiden Gruppen kaum unterschieden $(p=0,144)$. Insgesamt 10 Personen (davon 7 Atopiker) zeigten eine auffällige Lungenfunktion und 8 Personen (davon 6 Atopiker) einen positiven Methacholintest.

Obwohl überwiegend junge und nach eigenen Angaben nicht-allergische Personen an der Voruntersuchung teilnahmen, war es schwierig geeignete Studienteilnehmer zu finden. Fast die Hälfte der Untersuchten wies zum Teil deutliche Zeichen einer Atopie und $10 \%$ sogar eine bronchiale Hyperreaktivität auf. Ob diese Personen tatsächlich keine Anzeichen einer Allergie empfanden oder diese verschwiegen, um an der umfangreichen und mit einer Aufwandsentschädigung verbundenen Untersuchung teilzunehmen, kann abschließend nicht geklärt werden.

\section{P1.12}

\section{Allergengehalt in Birkenpollen - Eine Frage von Ort und Zeit}

\section{J. Zimmer, J. Kammler, S. Döring, D. Strecker, V. Mahler, S. Kaul}

\section{Paul-Ehrlich-Institut, Langen, Deutschland}

Birkenpollen bilden das biologische Ausgangsmaterial aller zurzeit auf dem Markt erhältlichen Produkte zur allergenspezifischen Immuntherapie (AIT) der Birkenpollenallergie. Wir konnten in früheren Arbeiten zeigen, dass ein schwankender Allergengehalt zwischen Chargen von AIT-Produkten mit unterschiedlichen in der Produktion eingesetzten Pollenchargen assoziiert sein kann. Darauf basierend untersuchen wir nun die Biodiversität der Birkenpollen genauer, um zu klären, ob über die Jahre und/oder zwischen Standorten Trends zu erkennen sind. Beispielsweise könnte ein genereller Anstieg des Allergengehalts in Birkenpollen sowohl in der Produktion als auch in der Regulation der AIT-Produkte zu Herausforderungen führen.

In einem über mehrere Jahre fortlaufenden Projekt haben wir Birkenpollen einzelner Bäume gesammelt und mittels allergenspezifischer ELISA-Systeme auf ihren Major- und Minorallergengehalt hin untersucht. Ergebnisse der ersten drei Jahre bestätigen eine große Variabilität: Der Gehalt einzelner Allergene im Pollen desselben Baumes ändert sich von Jahr zu Jahr und verschiedene Bäume zeigen deutliche Unterschiede im Allergengehalt ihrer Pollen, auch wenn sie in einem Umkreis von nur wenigen Metern gepflanzt wurden. Auf Basis dieser noch vorläufigen und lokal begrenzten Daten ist es aber nicht mög- 
lich, potentielle Trends, wie etwa einen sukzessiven Anstieg des Allergengehalts, abzuleiten. Daher haben wir in einem zweiten Projekt begonnen, eine große Anzahl kommerziell erhältlicher Pollenchargen der vergangenen 10 Jahre vergleichend zu analysieren. Die Birkenpollen stammen aus Tschechien, Frankreich und Schweden, wobei Ort und Datum der Sammlung jeweils dokumentiert ist. Vorläufige Ergebnisse zeigen, wie erwartet, deutliche Unterschiede zwischen Sammlungsorten und Jahren, weisen aber bisher nicht auf einen generellen Anstieg des Major- oder Minorallergengehalts der Birkenpollen in den letzten 10 Jahren hin.

\section{Food allergy}

\section{P2.1}

\section{Alpha-Gal-Syndrom - Orale Provokations- testungen zur Überprüfung einer klinischen Relevanz der Sensibilisierung}

\section{S. Aurich, J. Simon, R. Treudler}

Klinik für Dermatologie, Venerologie und Allergologie, Leipziger Interdisziplinäres Centrum für Allergologie (LICA), Universitätsmedizin Leipzig, Leipzig, Deutschland

Hintergrund: Beim Galactose- $\alpha$-1,3-galactose-( $\alpha$-Gal) Syndrom kommt es zu einer verzögerten Allergie auf rotes Fleisch (und Cetuximab). Der Nachweis von sIgE gegen $\alpha$-Gal geht nicht notwendigerweise mit einer klinischen Relevanz einher, so dass orale Provokationstestungen (OPT) sinnvoll sind. Wir beschreiben hier Erfahrungen mit entsprechenden OPTs.

Methode: Acht Patienten (5 Frauen) mit sIgE ( $>0.35 \mathrm{kU} / \mathrm{l})$ gegen $\alpha$-Gal erhielten stationär eine offene OPT. Dokumentiert wurden Alter, atopische Komorbiditäten, anamnestische Symptome, sIgE (CAP-FEIA, Phadia), OPT Ausgang.

Ergebnisse: Die Patienten (Median 33 Jahre, Spannweite 16-63) hatten anamnestisch Reaktionen (Ring und Messmer/ RM) $2 \times$ Grad I, $5 \times$ Grad II und $1 \times$ Grad III. Zwei Patienten hatten eine Rhinokonjunktivitis allergica, je einer ein Asthma bzw. eine atopische Dermatitis. Nur ein Patient zeigte eine leichte Tryptase-Erhöhung. Das sIgE für $\alpha$-Gal lag im Median bei $17,77 \mathrm{kU} / 1(1,25-93,7)$. Sechs Patienten wurden mit Schweinenieren, zwei mit Lammfleisch, drei mit Rindfleisch, zwei mit Gelatine und zwei mit Schweinefleisch provoziert. 4/8 (anamnestisch $n=3$ mit RM Grad 2 und $n=1$ Grad 3) Patienten zeigten Symptome unter OPT, davon $n=3$ auf das Einzelallergen $(200 \mathrm{~g}$ Schweinenieren $n=2,200 \mathrm{~g}$ Rindfleisch $n=1$ ) und $n=1$ in Kombination mit einem Kofaktor (200 g Schweinenieren $+500 \mathrm{mg}$ ASS). Bei $n=1$ konnte ein anderes Allergen als Auslöser identifiziert werden. Objektive Zeichen umfassten: $n=3$ Urtikaria, $n=1$ RR-Abfall, subjektive Symptome: $n=3$ Missempfindungen, $n=3$ Gefühl der Schleimhautschwellung, $n=1$ Bauchschmerzen/ Magenkrämpfe, $n=1$ Meteorismus, $n=1$ Schwindel.

Kommentar: Nur bei $50 \%$ der Patienten ließ sich eine klinische Relevanz der $\alpha$-Gal-Sensibilisierung mittels OPT bestätigen. Die Testung von Schweinenieren, ggfs mit Kofaktoren, war am häufigsten positiv, was sich auf den im Vergleich zu den anderen Provokationsmahlzeiten hohen Gehalt an $\alpha$-Gal zurückführen lässt.

\section{P2.2}

Efficacy and safety of in peanut allergy: results from a Phase 3, randomized double-blind, placebo-controlled trial (PALISADE)

\section{K. Blümchen', S. Zielen', A. Vereda' ${ }^{2}$, H. Hecker', S. Jones ${ }^{3}$, A. Burks ${ }^{4}$, R. Zawadzki' ${ }^{2}$, T. Casale ${ }^{5}$, J. Hourihane ${ }^{6}$, G. du Toit ${ }^{7}$, W. Shreffler ${ }^{8}$, B. Vickery ${ }^{9}$, D. Adelman ${ }^{2,}{ }^{10}$, K. Beyer ${ }^{11}$}

${ }^{1}$ Department for Children \& Adolescents, Division of Allergology, Pulmonology \& Cystic Fibrosis, Children's Hospital, Goethe University, Frankfurt, Germany

${ }^{2}$ Aimmune Therapeutics, Brisbane, California, United States 3Department of Pediatrics, University of Arkansas for Medical Sciences and Arkansas Children's Hospital, Little Rock, Arkansas, United States

${ }^{4}$ Department of Pediatrics, University of North Carolina, Chapel Hill, North Carolina, United States

${ }^{5}$ University of South Florida Morsani College of Medicine, Tampa, Florida, United States

'University College Cork, Cork, Ireland

${ }^{7}$ King's College London, London, United Kingdom

${ }^{8}$ Massachusetts General Hospital, Boston, Massachusetts,

United States

${ }^{9}$ Emory University School of Medicine, Atlanta, Georgia, United States

${ }^{10}$ University of California San Francisco, San Francisco, California, United States

${ }^{11}$ University Hospital Charité, Berlin, Germany

Introduction: Peanut allergy is one of the most common causes of food-related anaphylaxis and is characterized by a high unmet need for treatments that reduce risk of allergic reactions following accidental exposures. AR101 is a novel oral immunotherapy designed to address this unmet need.

Methods: PALISADE was a randomized, double-blind, placebo-controlled, international, phase 3 trial of AR101 in peanut-allergic subjects aged 4-55 years. Eligible subjects reacted at $\leq 100 \mathrm{mg}$ of peanut protein during double-blind, placebocontrolled food challenge (DBPCFC) at screening. Subjects completed initial escalation and up-dosing phases, $\sim 6$ months of $300 \mathrm{mg} /$ day maintenance, and an exit DBPCFC. The primary analysis, presented here, was conducted for subjects aged 4-17 years.

Results: Of 750 screened primary analysis subjects, 496 were randomized (AR101 $n=372$, placebo $n=124$ ) and received $\geq 1$ study dose. Of these, $66 \%$ were $4-11$ years old, $34 \% 12-17$ years old; $57 \%$ male; $72 \%$ had history of peanut anaphylaxis; $53 \%$ had history of asthma; $66 \%$ reported multiple food allergies. At baseline, median (range) maximum tolerated peanut dose was 10 (3-30) mg; median (IQR) peanut skin-prick wheal diameter was 11 (9-15) mm; $412(83 \%)$ completed PALISADE ( $80 \%$ AR101, 94\% placebo). Percentages of primary analysis subjects tolerating highest exit DBPCFC doses of 300, 600 (primary endpoint), and $1000 \mathrm{mg}$ were $77 \%, 67 \%$, and $50 \%$ for AR101 subjects, vs $\mathbf{8 \%}, 4 \%$, and $2 \%$ for placebo, respectively (primary endpoint between-group difference: $63.2 \%[P<0.00001 ; 95 \% \mathrm{CI}$ : 53.0-73.3]). No deaths or life-threatening adverse events (AEs) occurred. Among AR101 subjects, discontinuations occurred in $6.7 \%$ for gastrointestinal AEs and $2.7 \%$ following hypersensitivity reactions vs none for placebo.

Conclusions: These data, from the largest peanut allergy trial ever conducted, suggest AR101 is a potentially useful treat- 
ment in reducing clinical reactivity to peanut in highly sensitized peanut-allergic adults and adolescents.

\section{P2.3}

\section{Wide variability in terminology used in oral} immunotherapy: results from a diverse sample of US-based allergists and immunologists

\section{H. Hecker', B. Chipps'2, C. Ciaccio ${ }^{3}$, K. Rosén', T. Haselkorn', E. Zigmont', T. Casale ${ }^{5}$}

${ }^{1}$ Aimmune Therapeutics, Brisbane, California, United States ${ }^{2}$ Capital Allergy \& Respiratory Disease Center, Sacramento, California, United States

${ }^{3}$ The University of Chicago Medicine, Chicago, Illinois, United States

${ }^{4}$ EpiMetrix, Inc., Los Altos, California, United States

${ }^{5}$ Division of Allergy and Immunology, University of South

Florida, Tampa, Florida, United States

Introduction: The terminology used in food oral immunotherapy (OIT) varies globally. Given promising phase 3 results, OIT may soon become first-line treatment for peanut allergy, and a standard lexicon is needed. Real-world data were collected from a diverse sample of US allergists/immunologists to better understand the preferred terminology utilized in OIT, particularly when communicating with patients.

Methods: An online, self-administered survey was fielded to US allergists and/or immunologists between December 28, 2017 and January 27, 2018. Eligible participants completed training $\geq 5$ years ago, spent $>20 \%$ of their time in direct patient care, and managed $\geq 50$ patients with food allergy.

Results: Of 101 total participants from 28 US cities, $48 \%$ had administered OIT in the past year. Of these, $46 \%$ were practicing in or affiliated with an academic medical center, $47 \%$ had been in practice for $11-20$ years, and $78 \%$ spent $81-100 \%$ of their time in direct patient care. Respondents' definitions for "reactive dose" during oral food challenge (OFC) varied as follows: "dose at which symptoms occur" (63\%); "lowest dose at which symptoms occur" (14\%); and "cumulative dose at which symptoms occur" (10\%). Respondents' definitions of "tolerated dose" during OFC similarly varied: "highest dose tolerated without symptoms" (32\%); "dose at which no symptoms occur" (30\%); and "cumulative dose tolerated without symptoms" (11\%). After OIT, $81 \%$ of participants identified "tolerated dose" as most clinically meaningful for patients to understand; $88 \%$ said a stronger consensus on OIT terminology would be extremely/ very useful. A similar number felt that educating patients on the specific terminology would also be useful.

Conclusions: OIT terminology varies considerably and a standardized lexicon is important for successful implementation, patient education, and safety. "Tolerated dose" was considered by physicians to be the most clinically relevant term for patients.

\section{P2.4}

Vogel, Ei und Chicken Nuggets allergische Berufserkrankung bei einem Patienten mit Vogel-Ei-Syndrom

\section{A. J. Kilbertus, W. Hötzenecker, B. Zahel, I. Angelova-Fischer}

Dermatologie, Kepler Uniklinikum Linz, Linz, Österreich

Beim Vogel-Ei-Syndrom handelt es sich um eine IgE-vermittelte Kombination aus Inhalations- und Nahrungsmittel-Allergie. Abgesehen von Reaktionen auf die orale Zufuhr kann die Inhalation von Ei-Protein am Arbeitsplatz bei sensibilisierten Patienten respiratorische Beschwerden und dadurch auch berufliche Einschränkungen verursachen.

Ein 30-jähriger Patient stellte sich in unserer Allergieambulanz aufgrund Rhinitis und Atemnotattacken am Arbeitsplatz vor. Er arbeitete in einer Nahrungsmittel-Fertigungshalle eines Fastfood-Herstellers, wo er mit Mundschutz und Handschuhen seiner Tätigkeit in der Befüllung von Maschinen mit PanierMehl für Chicken Nuggets nachging. Eine Typ-I-Sensibilisierung gegen klassische Inhalationsallergene war nicht vorbekannt, in der Kindheit wurden Wellensittiche als Haustiere gehalten. In der allergologischen Anamnese ließ sich eine Unverträglichkeit von gekochtem Ei mit Übelkeit, Erbrechen und Schwindel eruieren. Im Rahmen der PRICK Testung mit den suspizierten Substanzen kam es zu einer anaphylaktischen Reaktion zweiten Grades. In der Testung zeigte sich eine ++ positive Reaktion auf Eiweiß und Eigelb sowie eine +++ positive Reaktion auf das beruflich exponierte Panier-Mehl. Letzteres beinhaltete neben diversen Mehlen und Weizenstärke auch einen erheblichen Anteil an Ei-Albumin. In der ergänzenden in-vitro-Diagnostik zeigten sich positive spezifische IgE gegen Gesamt-Ei, HühnerEiweiß, nGal d1 nGal d2, nGal d5 sowie gegen Vogelfedern, die die klinische Verdachtsdiagnose eines Vogel-Ei-Syndroms bestätigte. Die Abfolge der Beschwerden veranschaulicht den Weg der primär inhalativen Sensibilisierung im Kindesalter mit folglicher symptomatischer Nahrungsmittelallergie und respiratorischer Symptomatik über das in Ei und Panier-Mehl vorhandene kreuzreaktive Allergen Alpha-Livetin (Gal d5). Eine Versetzung innerhalb des Betriebes in die Rindfleisch-Abteilung ermöglichte dem Patienten eine Beschwerdefreiheit am Arbeitsplatz.

\section{P2.5}

Untersuchungen zum Einfluss des

Polyphenolgehaltes auf die in-vitro-Allergenität alter und neuer Apfelsorten - eine Pilotstudie

\section{J. Kschonsek ${ }^{1}$, C. Wiegand ${ }^{2}$, U. Hipler², V. Böhm¹}

${ }^{1}$ Institut für Ernährungswissenschaften, Friedrich-SchillerUniversität, Jena, Deutschland

${ }^{2}$ Klinik für Hauterkrankungen, Universitätsklinikum Jena, Jena, Deutschland

Hintergrund: In den vergangenen Jahrzehnten ist die Birkenpollen-assoziierte Apfelallergie mit rund vier Millionen Betroffenen die wichtigste Fruchtallergie in Deutschland geworden. Diese steigende Anzahl von Allergikern wird mit einem höheren Konsum von neuen Apfelsorten in Verbindung 
gebracht. So wird vermutet, dass durch Züchtung neuer Sorten mit reduziertem Polyphenolgehalt die erhöhte Allergenität verursacht wird.

Methode: Der Polyphenolgehalt von zwei alten (Ontario, Dülmener Rosenapfel) und zwei neuen (Braeburn, Granny Smith) Apfelsorten wurde mittels HPLC analysiert. In einer Pilotstudie wurde die Konzentration von Sulfidoleukotrienen (CAST) und die CD63 Basophilenaktivierung (BAT) im Blut von 27 Birkenpollen-assoziierten Apfelallergikern nach Inkubation mit den o.g. Sorten bestimmt. Zusätzlich wurde der Grad der enzymatischen Bräunung photometrisch ermittelt.

Ergebnisse: Das Fruchtfleisch der alten Apfelsorten hatte einen signifikant $(p<0,001)$ höheren Polyphenolgehalt $(86 \pm 6 \mu \mathrm{g} / \mathrm{g})$ im Vergleich zu den Neuen $(25 \pm 7 \mu \mathrm{g} / \mathrm{g})$. Die alten Apfelsorten zeichneten sich vor allem durch einen hohen Anteil an Chlorogensäure (63\%) aus, während der Gehalt in den neuen Sorten 14-mal niedriger war. Die Konzentration von Sulfidoleukotrienen sowie die CD63 Basophilenaktivierung der alten Apfelsorten war bis zu $62 \%$ niedriger gegenüber den Neuen und nahm mit steigendem Grad an enzymatischer Bräunung ab. Korrelationsanalysen zeigten, dass die in-vitro-Tests signifikant $(p<0,01)$ mit dem Polyphenolgehalt korrelierten (CAST: $r=-0,547$; BAT: $r=-0,639$ ), wobei vor allem der Gehalt an Chlorogen- und Kaffeesäure den stärksten Einfluss auf die in-vitro-Allergenität von Äpfeln hatte.

Schlussfolgerung: Alte Apfelsorten wie Ontario und Dülmener Rosenapfel könnten somit besser von Birkenpollenassoziierten Apfelallergikern toleriert werden als neue Sorten, da sie einen höheren Polyphenolgehalt sowie eine stärkere enzymatische Bräunung besitzen.

\section{P2.6}

IgE-vermittelte Ko-Faktor verstärkte Anaphylaxie auf Gerstenmalz

\section{S. Mühlenbein, J. Pickert, C. Möbs, W. Pfützner}

Klinik für Dermatologie und Allergologie Marburg, UKGM, Marburg, Deutschland

Bei einem 51jährigen Patienten kam es nach dem Genuss von Fleischkäse, Senf, Brot und Radler zu Urtikaria und Angioödem mit Schluckbeschwerden. An Vorerkrankungen bestand u. a. eine Gräserpollen- und Mehlstauballergie sowie ein Asthma bronchiale. Im Pricktest zeigten sich positive Reaktionen auf das Radler, Gerstenmalzschrot, verschiedene Biersorten wie Keller-, Malz-, Pils, Weizen- und alkoholfreies Bier. Keine Reaktionen fanden sich auf Hopfen, Bierhefe und Ethanol. Spezifische IgE-Antikörper waren gegen Gerstenmehl (CAP Klasse 3) sowie verschiedene Aeroallergene, nicht jedoch gegen Senf, Galaktose-Alpha-1,3-Galaktose, Omega-5-Gliadin und die Lipid-Transfer-Proteine Tri a 14 und Pru p 3 nachweisbar. Im Basophilenaktivierungstest fand sich eine konzentrationsabhängige positive Reaktion auf Braugerste mit max. $21.9 \%$ aktivierten CD63+ Basophilen bei einer Verdünnung von 1:34 (Pos.-Kontrollen: unspezifisch 24,2\%, spezifisch 20,4\%) bei negativem Befund für Hopfen, Ethanol und Essigsäure.

Es erfolgte ein doppelblinder, placebokontrollierter oraler Provokationstest mit Gerstenmalz sowie mit alkoholfreiem und alkoholhaltigem Bier. Während Gerstenmalz in Form von Gerstenschrot (max. Einzelmenge $132 \mathrm{~g}$, max. kumulative Menge $200 \mathrm{~g}$ ) sowie alkoholfreies Bier (max. Einzelmenge $330 \mathrm{ml}$, kumulative Tagesmenge $600 \mathrm{ml}$ ) zu subjektiven Beschwerden in Form von enoralem Juckreiz führten, kam es nach Konsum von $330 \mathrm{ml}$ alkoholhaltigem Pils Bier zu einer generalisierten
Urtikaria, Angioödemen und Dyspnoe. Serologisch fand sich ein Anstieg der Mastzelltryptase von 3,9 $\mu \mathrm{g} / \mathrm{l}$ (Basiswert) auf $8,9 \mu \mathrm{g} / \mathrm{l}$.

Zusammenfassend konnten wir eine IgE-vermittelte Ko-Faktor induzierte Anaphylaxie auf Gerstenmalz diagnostizieren, wobei alkoholhaltiges Bier sowohl als Allergenträger als auch als Augmentationsfaktor fungierte.

\section{P2.7}

\section{Verträglichkeit von Dinkel bei Weizenallergie - ein Fallbericht}

\section{S. Schwarz ${ }^{1}$, S. Albroscheit ${ }^{1}$, J. Dobbertin-Welsch ${ }^{2}$, S. Yürek ${ }^{2}$, V. Trendelenburg' ${ }^{2}$, S. Lau², K. Beyer ${ }^{2}$}

${ }^{1}$ Kinderklinik, Charité Universitätsmedizin Berlin, Berlin, Deutschland

${ }^{2}$ Charité Universitätsmedizin Berlin, Berlin, Deutschland

Hintergrund: Die Weizenallergie gehört zu den häufigsten Nahrungsmittelallergien im Säuglings- und Kleinkindalter. Im Fall einer diagnostizierten Allergie auf Weizen (Triticum aestivum) wird oft eine generelle Eliminationsdiät für alle weizenverwandten Getreidesorten wie Dinkel (Triticum spelta) empfohlen. Erfahrungsgemäß ist die Wahrscheinlichkeit der Kreuzreaktivität von Dinkel bei Weizenallergikern sehr hoch. Es gibt jedoch auch Ausnahmen.

Fallbeispiel: Anamnestisch kam es bei einem 11 Monate alten Jungen 30 min nach Verzehr eines milchfreien Weizengrießbreies zu einer generalisierten Urtikaria. In der Allergiediagnostik fand sich spezifisches IgE auf Weizen $(0,31 \mathrm{kU} / \mathrm{l})$, Hühnerei $(0,97 \mathrm{kU} / \mathrm{l})$ und Kuhmilch $(1,30 \mathrm{kU} / \mathrm{l})$. Mittels doppeltblind, Plazebo kontrollierter oraler Nahrungsmittelprovokation wurde bei uns mit 15 Monaten eine Kuhmilch-, Hühnerei- und Weizenallergie diagnostiziert. In Bezug auf Weizen trat eine Reaktion nach der 4. Gabe (0,14 g Weizengluten, 0,112 g Protein) mit generalisierter Urtikaria auf. Da Dinkel jedoch anamnestisch in kleinen Mengen verzehrt und vertragen wurde, führten wir zur abschließenden Sicherung der Verträglichkeit eine offene Nahrungsmittelprovokation mit Dinkel durch. Alle Gaben wurden gut vertragen. Es erfolgte eine entsprechende Ernährungsberatung mit der Empfehlung zur regelmäßigen Gabe von Dinkel bei gleichzeitiger Meidung von Weizen.

Fazit: Dies ist der uns erste bekannte Fall eines Weizenallergikers, der nachweislich Dinkel verträgt. Da generell bei Weizenallergikern aufgrund der Kreuzreaktivität keine Nahrungsmittelprovokation mit Dinkel durchgeführt wird, könnte es sein, dass noch mehr Patienten mit Weizenallergie Dinkel vertragen. Angesichts der Tatsache, dass kostenintensive glutenfreie Backwaren oft ein ungünstiges Nährstoff- und Ballaststoffprofil aufweisen und glutenfreie Mehle häufig eine schlechte Backeigenschaft besitzen, könnten solche Kinder Dinkelprodukte als bessere Alternative zu glutenfreien Produkten nutzen. 
P2.8

\section{Development of a recombinant vaccine based on virus-like particles for the treatment of peanut allergy}

\section{F. Storni', A. Zeltins' ${ }^{2}$, M. Heath ${ }^{3}$, M. Kramer ${ }^{4}$, M. Skinner ${ }^{3}$, L. Zha' ${ }^{1}$, E. Rösti' ${ }^{1}$, P. Engeroff', M. Mohsen ${ }^{1}$, M. Vogel' ${ }^{1}$, T. Kündig ${ }^{5}$, M. Bachmann'}

${ }^{1}$ Immunology, RIA, Inselspital, Bern, Switzerland ${ }^{2}$ Latvian Biomedical Research and Study Centre (BRSC), Riga, Latvia

${ }^{3}$ Allergy Therapeutics, Worthing, United Kingdom

${ }^{4}$ Bencard Allergie GmbH, Munich, Germany

${ }^{5}$ Dermatology, University Hospital Zürich, Zurich, Switzerland

Introduction: Peanuts harbour at least 12 different antigens responsible for allergy induction in humans. The major allergens Ara $\mathrm{h} 1$ and Ara $\mathrm{h} 2$ are recognised by IgE from more than $95 \%$ of peanut-sensitive patients. To treat peanut allergy we displayed Ara h 1 and Ara h 2 on virus-like particles (VLPs) derived from plant viruses and tested their safety and efficacy pre-clinically.

Methods: Ara h 1 was purified from extracts while Ara h 2 was produced recombinantly. Both allergens were separately coupled to VLPs and used to immunise mice previously sensitised to peanut-extract. Allergic responses were measured by skin prick tests, intestinal inflammation upon oral and temperature drop upon intravenous challenge.

Results: We demonstrated that both allergens induce strong and protective IgG responses in peanut allergic mice and ameliorate local allergic symptoms in skin-prick-tests and allergen induced gut-inflammation. Systemic symptoms upon intravenous challenge with allergen-extract were also strongly suppressed. Despite strongly enhanced immune responses induced by allergens displayed on VLPs, the allergens were fully detoxified as they failed to trigger an allergic response in sensitised mice and failed to activate basophils of allergic individuals.

Conclusions: Vaccination against either Ara h 1 or Ara $h$ 2 alone was sufficient to induce protection against the whole extract consisting of multiple allergens. We will elucidate the mechanism of this clinically highly relevant phenomenon.

\section{P2.9}

Schwellenwert, Organbeteiligung und Schweregrad unter wiederholter Nahrungsmittelprovokation sind häufig nicht reproduzierbar

V. Trendelenburg ${ }^{1}$, M. Veit ${ }^{1}$, T. Keil ${ }^{2}$, L. Lange ${ }^{3}$, S. Büsing ${ }^{4}$, C. Kemen ${ }^{5}$, F. Friedrichs ${ }^{6}$, S. Scheewe ${ }^{7}$, P. Stock ${ }^{8}$, R. Schlags ${ }^{9}$, V. Stephan ${ }^{10}$, M. Gappa ${ }^{11}$, J. Seidenberg ${ }^{12}$, M. Millner-Uhlemann ${ }^{13}$, H. Ott ${ }^{14}$, M. Barker ${ }^{15}$, B. Niggemann', K. Beyer ${ }^{1}$

${ }^{1}$ Klinik für Pädiatrie mit Schwerpunkt Pneumologie, Immunologie und Intensivmedizin, CharitéUniversitätsmedizin, Berlin, Deutschland

${ }^{2}$ Institut für Sozialmedizin, Epidemiologie und

Gesundheitsökonomie, Charité-Universitätsmedizin, Berlin, Deutschland

${ }^{3}$ Kinderklinik, St. Marien-Hospital, Bonn, Deutschland ${ }^{4}$ Zentrum für Kinder- und Jugendmedizin, Christliches Kinderhospital, Osnabrück, Deutschland

${ }^{5}$ Katholisches Kinderkrankenhaus Wilhelmstift, Hamburg, Deutschland

${ }^{6}$ Kinderarztpraxis Laurensberg, Aachen, Deutschland

${ }^{7}$ Fachklinik Sylt, Westerland, Deutschland

${ }^{8}$ Altonaer Kinderkrankenhaus, Hamburg, Deutschland ${ }^{9}$ Klinik für Pädiatrische Pneumologie und Allergologie, Fachklinik Wangen, Wangen, Deutschland

${ }^{10} \mathrm{Klinik}$ für Kinder- und Jugendmedizin, Sana Klinikum Lichtenberg, Berlin, Deutschland

${ }^{11} \mathrm{Klinik}$ für Kinder- und Jugendmedizin, Marien-Hospital $\mathrm{GmbH}$, Wesel, Deutschland

${ }^{12}$ Elisabeth-Kinderkrankenhaus, Klinikum Oldenburg, Medizinischer Campus, Carl von Ossietzky Universität Oldenburg, Oldenburg, Deutschland

${ }^{13}$ Kinderallergologiepraxis, Stuttgart, Deutschland

${ }^{14}$ Kinder- und Jugendkrankenhaus, Hannover, Deutschland

${ }^{15}$ Klinik für Kinder- und Jugendmedizin, Helios Klinikum Emil von Behring, Berlin, Deutschland

Hintergrund: Bisher gibt es nur wenige publizierte Daten bezüglich der Veränderungen bzw. Reproduzierbarkeit des Schwellenwertes sowie der Organbeteiligung und des Schweregrads bei wiederholter allergischer Reaktion bei Kindern mit Nahrungsmittelallergien. Ziel der Studie war es daher, diese drei Charakteristika allergischer Reaktionen unter wiederholter Nahrungsmittelprovokation systematisch $\mathrm{zu}$ untersuchen.

Methoden: Kinder mit einer positiven Erstprovokation (EP) und Reprovokation (RP) auf Hühnerei $(n=96)$, Kuhmilch $(n=46)$ oder Erdnuss $(n=25)$ wurden in die Auswertung eingeschlossen. Der Abstand zwischen beiden Provokationen betrug mindestens 6 Monaten, währenddessen wurde eine entsprechende Eliminationsdiät durchgeführt. Die Provokation erfolgte gemäß Leitlinien in 7 halblogarithmischen Titrationsstufen sowie, bei Ausbleiben von allergischen Reaktionen, einer Kumulativgabe. Der Schweregrad der Reaktionen wurde auf einer Skala von I-V bewertet.

Ergebnisse: Insgesamt zeigte sich im Median ein Schwellenwert-Anstieg für objektive Reaktionen zwischen $\mathrm{EP}$ und RP für Hühnerei $(516 \mathrm{mg}$ vs. $1290 \mathrm{mg}$ Protein, $p<0,001$ ), Kuhmilch (340 mg vs. $1020 \mathrm{mg}$ Protein, $p<0,05)$ und Erdnuss ( $300 \mathrm{mg}$ vs. $1000 \mathrm{mg}$ Protein, $p=$ n.s.). Jedoch kam es intraindividuell $\mathrm{zu}$ teilweise gravierenden Unterschieden mit 
Erhöhung oder Absinken des Schwellenwertes um bis zu 4 Titrationsstufen. Der Schweregrad der Reaktion im Rahmen der RP wich verglichen mit der EP bei $69 \%$ der Hühnerei-, $61 \%$ der Erdnuss- und $55 \%$ der Kuhmilch-allergischen Kinder um mindestens eine bis maximal 3 Skalenstufen ab. Die Organbeteiligung allergischer Reaktionen ließ sich bei der RP häufig nicht reproduzieren, vor allem hinsichtlich des Respirationstrakts.

Zusammenfassung: Individueller Schwellenwert, Organbeteiligung und Schweregrad einer allergischen Reaktion können sich im Zeitverlauf gravierend verändern. Eine individuelle Beratung und Prognose anhand einer oralen Provokation ist daher nur eingeschränkt möglich.

\section{P2.10}

Mögliche Behandlung des Oralen Allergie Syndroms durch Birken AIT - eine Studie zur Untersuchung des Effektes der SQ Frühblüher SLIT-Tablette auf Symptome einer Apfel-Provokation

\section{R. Treudler ${ }^{1}$, S. Jacobsen ${ }^{2}$, N. Ghaussy ${ }^{3}$, H. Wolf ${ }^{3}$, T. Biedermann ${ }^{4}$}

${ }^{1}$ Klinik und Poliklinik für Dermatologie, Venerologie und Allergologie, Universitätsklinikum Leipzig, Leipzig, Deutschland

${ }^{2}$ Global Pharmacovigilance \& Clinical Development, ALK A/S, Hørsholm, Dänemark

${ }^{3}$ Clinical Operations, ALK-Abelló Arzneimittel GmbH, Hamburg, Deutschland

${ }^{4}$ Klinik für Dermatologie und Allergologie am Biederstein, TU München, München, Deutschland

Hintergrund: Viele Baumpollenallergiker entwickeln lokale Symptome gegen bestimmte Nahrungsmittel wie rohes Gemüse, Früchte und Nüsse (Orales Allergies Syndrom, OAS). Die AIT mit Birken-Allergen könnte sich positiv bei der Behandlung des OAS aufgrund der Kreuzreaktivität zwischen Bet v 1 und den OAS auslösenden Bet v 1-homologen Allergenen auswirken.

Methoden: Die Studie TT-04 war eine randomisierte, DBPC Phase III-Studie zur Wirksamkeit und Sicherheit der SQ Frühblüher SLIT-Tablette bei Rhinokonjunktivits gegen Pollen der birkenhomologen Gruppe. 634 Patienten wurden 1:1 für die SQ Frühblüher-Tablette (Dosis 12 DU) oder Placebo randomisiert. Eine Subgruppe mit OAS $(N=124)$ nahm an einem offenen Apfelprovokationstest (APT) bei Studienende mit Einnahme ansteigender Apfelmengen (4, 8, 16, 32, 64 g Apfel) im Abstand von 15 Minuten teil. Ausgewertet wurden die OAS-Symptome (VAS) und die globale Wirksamkeit auf das OAS (Verbesserung seit Einschluss) sowie apfel-spezifische Mal d 1-Antikörper im Verlauf der Studie gemessen.

Ergebnisse: Die OAS-Symptomscores waren nach APT in beiden Behandlungsgruppen generell niedrig (VAS $\leq 1 \mathrm{~cm}$ ). Placebo-Patienten hatten einen deutlicheren Anstieg der Symptome nach dem 1. APT gegenüber der aktiven Gruppe, in der mehr Patienten über eine Verbesserung der OAS-Symptome nach Behandlung gegenüber Placebo (87\% vs. $64 \%$; OR $0,27 ; p=0,0028)$ berichteten. Unter aktiver Behandlung stieg der Serumspiegel für Mal d 1-spezifisches IgE und IgG4 mit der gleichen Kinetik wie für birkenspezifisches IgE und IgG4 an. Es traten keine lokalen oder systemischen Reaktionen während des APT auf.
Schlussfolgerung: Zusammengenommen zeigen die Daten des APT in der Studie TT-04 einen Trend für einen Effekt der Behandlung mit der SQ Frühblüher SLIT-Tablette auf die OASSymptome. Zudem wurde ein Anstieg der apfel-spezifischen Antikörper beobachtet, was andeutet, dass die SQ Frühblüher SLIT-Tablette eine Toleranz gegenüber Apfel induzieren könnte.

\section{P2.11}

\section{Anaphylaxie in der Sushi-Bar}

S. van Dullemen ${ }^{1}$, M. Dressler ${ }^{1}$, A. Fischl' ${ }^{1}$, K. Steffen ${ }^{2}$, P. Rosengren ${ }^{3}$, K. Blümchen ${ }^{1}$

${ }^{1}$ Pädiatrische Pneumologie, Allergologie und Mukoviszidose, Universitätsklinik Frankfurt, Goethe Universität, Frankfurt/ Main, Deutschland

${ }^{2}$ ImmunoDiagnostics, Thermo Fisher Scientific, Thermo Fisher Diagnostics GmbH, Freiburg, Deutschland ${ }^{3}$ ImmunoDiagnostics, Thermo Fisher Scientific, Uppsala, Schweden

Hintergrund: Zunehmende kulturelle Einflüsse aus dem Ausland stellen Allergologen sowie allergologisch versierte Ernährungsfachkräfte vor neue Herausforderungen.

Kasuistik: Stationäre Aufnahme eines $1 \mathrm{Jahr} 7$ Monate alten, japanischen Mädchens wegen einer anaphylaktischen Reaktion (generalisierte Urtikaria, Heiserkeit, Erbrechen) nach 5-30 Minuten nach dem Verzehr von Gurke, Essig, Misosuppe (Soja), Ikura (Lachsrogen), Sesam, Buchweizen, Muscheln, Shrimps und Senf in einem japanischen Restaurant. Milch, Soja, Sesam, Buchweizen und Hühnerei würden vertragen, Muscheln und Senf seien noch nie verspeist worden. Der Verzehr von Shrimps sei unproblematisch, nach Hautkontakt allerdings Augenschwellung.

Befunde: IgE-Testung: Negativ für Lieschgras, Roggen, Birke, Katze, Pferd, Milch, Dorsch, Lachs, Thunfisch, Pinienkerne, Walnuss, Mandel, Cashew, Krabbe, Garnele, Muschel, Anasakis, Erdnuss.

Senfsaat 0,29 kU/1, Dermatophagoides farinae 2,7 kU/1, Hühnerei $5,9 \mathrm{kU} / \mathrm{l}$.

Ambulanter Prick-to-Pricktest: Histamin 4 mm, $\mathrm{NaCl} 0$ mm, Shrimps/Lachs $0 \mathrm{~mm}$, Lachsrogen $13 \mathrm{~mm}$.

Bestätigung: IgE gegen Lachsrogen (Spezialtestung für seltene Allergene, ImmunoCAP ${ }^{\circledR}$ nur für Forschungszwecke, durch Thermo Fisher Scientific, Uppsala, Schweden): IgE 2,5 kU/l.

Diskussion: Bei eindeutiger Anaphylaxie und passender Sensibilisierung ist eine Lachsrogen-Allergie sehr wahrscheinlich. In der traditionellen japanischen Küche werden Fischrogen in Form von Sushi und Ikura-Gerichten täglich serviert. Die Häufigkeit der Fischrogen-Allergie in Japan liegt bei 3,7 \% und steht somit an sechster Stelle der häufigsten Nahrungsmittelallergien. Weil eine Deklarationspflicht für Lachsrogen fehlt und diese in japanischen Restaurants nicht strikt gemieden werden können, erhielt das Kind ein Notfallset mit Adrenalin-Autoinjektor. 
P2.12

\section{Sicherheitsaspekte bei der Verwendung von Magermilchpulver für die orale Kuhmilchprovokation - ein Fallbericht}

\section{S. Yürek, S. Albroscheit, V. Trendelenburg, K. Beyer}

Charité Universitätsmedizin Berlin, Berlin, Deutschland

Hintergrund: Orale Nahrungsmittelprovokationen stellen den Goldstandard in der Diagnostik der Nahrungsmittelallergie dar. Bezüglich der Zubereitung der Allergene gibt es jedoch bisher keinen einheitlichen Konsensus und die verwendeten Materialien unterscheiden sich häufig von Klinik zu Klinik. Der folgende Fallbericht soll dieses Problem in Bezug auf die Verwendung von Milchpulver zur Diagnostik der Kuhmilchallergie beleuchten.

Fallbericht: Bei einem 9 Monate alten männlichen Säugling wurde eine klinisch indizierte Kuhmilchprovokation durchgeführt. Da das Kind auch an einer internationalen Studie teilnahm, erfolgte die Kuhmilchprovokation zur besseren Vergleichbarkeit mit Magermilchpulver. Aufgrund einer nicht eindeutigen Reaktion mit flüchtiger perioraler Rötung nach der Kumulativdosis wurde diese mit 3,8 \%iger pasteurisierter, traditionell verarbeiteter Kuhmilch wiederholt. Sofort nach dem Verzehr von etwa 2 Teelöffeln zeigte der Patient zunächst eine periorale Rötung. Nach 5 Minuten kamen Hauterythem und Pruritus an Gesicht und Stamm sowie Urtikaria am Oberkörper hinzu. Die Symptome waren nach oraler Gabe von Antihistaminika rückläufig.

Klinische Relevanz des Fallberichts: Milchpulver bietet zwar die Möglichkeit einer Standardisierung der Allergenzubereitung für die orale Nahrungsmittelprovokation, scheint aber durch die Verarbeitung nicht das gesamte Allergenspektrum von Frischmilch zu haben. Ein Patient, der in einer mit Milchpulver durchgeführten Nahrungsmittelprovokation keine Reaktion zeigt, sollte anschließend in der Klinik Frischmilch erhalten, um das Risiko einer Reaktion auf diese auszuschließen.

\section{P2.13}

\section{Retrospektive Analyse von 10 Jahren Nahrungsmittelprovokation}

\section{N. Friedrich, K. Nemat, C. Vogelberg}

Klinik und Poliklinik für Kinder- und Jugendmedizin, Fachbereich Kinderpneumologie/Allergologie, Universitätsklinikum Carl Gustav Carus, Dresden, Deutschland

Hintergrund: Orale Nahrungsmittelprovokationen gelten als Goldstandard in der Diagnostik von Nahrungsmittelallergien.

Methoden: Bei 420 Kindern (Median 3,9 Jahre) wurden über einen Zeitraum von 10 Jahren 860 orale Provokationen durchgeführt. Die Daten wurden retrospektiv anhand der Provokationsprotokolle erfasst und nach Vorhersagewerten eines positiven Testergebnisses gesucht.

Ergebnisse: Die am häufigsten provozierten Nahrungsmittel waren Hühnerei $(34,3 \%)$, Kuhmilch $(20,8 \%)$, Erdnuss $(11,7 \%)$ und Baumnüsse (11,6 \%).
Unterschiede der benötigten Verummenge bis zum Auftreten von Symptomen zeigten sich zwischen den Schweregradgruppen nicht. Für keines der Allergene konnte ein signifikanter Zusammenhang zwischen der Ausprägung der nahrungsmittelspezifischen Sensibilisierung und dem Schweregrad der provozierten allergischen Reaktion nachgewiesen werden. Bei der Korrelation der Höhe des s-IgE-Titers mit der kumulativen Verummenge ergaben sich für Hühnereiweiß f1, Erdnuss f13, rAra h2 und rCor a1 nur mittelgradige inverse Korrelationskoeffizienten.

Für rAra h2 wurde eine gute Sensitivität von $92,7 \%$ bei gleichzeitig hoher Spezifität von 82,8 \% ermittelt ( $n=70$; Cut-off $0,70 \mathrm{kU} / \mathrm{l})$. Im Vergleich dazu lag die Spezifität für Erdnuss f13 bei nur $28,9 \%$.

Folgende „decision points“ für eine $99 \%$-ige Vorhersagewahrscheinlichkeit einer positiven Provokation konnten bestimmt werden: Hühnereiweiß f1 33,39 kU/l, Erdnuss f13 $61,92 \mathrm{KU} / \mathrm{l}$ und rAra h2 12,44 kU/l.

\section{Zusammenfassung:}

- Kein eindeutiger Zusammenhang zwischen Schweregrad oder Verummenge und Stärke der nahrungsmittelspezifischen Sensibilisierung

- „Decision points“ können populationsspezifisch genutzt werden, um Provokationen einzusparen

\section{Specific immunotherapy - clinical data}

\section{P3.1}

A pivotal phase III allergen immunotherapy study to assess the efficacy and safety of subcutaneously administered tyrosine adsorbed modified birch allergen + MPL

W. Aberer ${ }^{1}$, P. Kuna', L. Biermer ${ }^{3}$, O. Pfaar ${ }^{4}$, S. Zielen ${ }^{5}$, U. Berger ${ }^{6}$, D. Lee ${ }^{7}$, M. Seybold7 ${ }^{7}$ A. Ernst ${ }^{7}$, A. Clausen ${ }^{7}$, K. Lis ${ }^{7}$, A. Wittmann ${ }^{7}$, K. Gunawardena ${ }^{8}$, T. Higenbottam ${ }^{8}$, M. Skinner ${ }^{8}$

'Department of Dermatology, Medical University of Graz, Graz, Austria

${ }^{2}$ Barlicki University Hospital, Medical University of Lodz, Lodz, Poland

${ }^{3}$ Respiratory Medicine and Allergology, Lund University, Lund, Sweden

${ }^{4}$ Department of Otorhinolaryngology, Head and Neck Surgery, Universitätsmedizin Mannheim, Wiesbaden, Mannheim, Germany

${ }^{5}$ Medaimun GmbH, Frankfurt, Germany

${ }^{6}$ Research Group Aerobiology and Pollen Information, Department of Otorhinolaryngology, Vienna, Austria ${ }^{7}$ Bencard Allergie GmbH, Munich, Germany ${ }^{8}$ Allergy Therapeutics, Worthing, United Kingdom

Introduction: 2 previous dose-finding trials utilizing the Conjunctival Provocation Test (CPT) showed that a modified birch allergen subcutaneous immunotherapy (SCIT) product $(1.0 \mathrm{ml})$ with modified allergen tyrosine adsorbate (MATA) and monophosphoryl lipid A (MPL) adjuvants at a cumulative dose of $27.300 \mathrm{SU}$ was the most effective dose without added safety signals. The current pivotal Phase III study aims to confirm the safety and efficacy of this cumulative dose compared to placebo 
in patients with moderate to severe seasonal allergic rhinoconjunctivitis due to birch-pollen with or without mild asthma.

Methods: This multi-center (59 clinical study centers in 4 European countries) randomized, double-blind, placebo controlled, parallel-group study was initiated in 2017 . The primary outcome of this pivotal Phase 3 study is the difference in the EAACI recommended total Combined Symptom and Medication Score (CSMS; Pfaar 2014), collected once daily and averaged over the peak birch pollen season (BPS) of 2018, between the 27.300 SU and placebo treatment groups. The linguistic validity of the sponsor-developed CSMS questionnaire was ensured via intensive expert review and cognitive debriefing sessions with panels of allergy patients. Cooperation with the European Aeroallergen Network allowed for calculation of predicted and actual start and stop dates of the entire and peak BPS.

Results: In total, 944 pts were screened and 582 were randomized. The design and preliminary results of this study will be discussed with consideration of experience and results achieved in two related dose-finding studies utilizing CPT.

Conclusions: The achievement of the efficacy and safety objectives of a birch MATA MPL SCIT product at a cumulative dose of 27300 SU in this pivotal Phase III study will be an important milestone in the development of an efficacious and safe state-of-the-art birch SCIT, and provide further insight into the CPT dose-finding methodology applied to select this dose.

\section{P3.2}

Die Therapieallergene-Verordnung (TAV): Status und Entwicklungen bei der spezifischen Immuntherapie (SIT)

\section{Bachert ${ }^{1}$, A. Horn'², M. Wagenmann³, D. Brehmer², U. Wahn ${ }^{4}$}

${ }^{1}$ Abteilung für Hals-, Nasen, Ohrenheilkunde, Universität Gent, Gent, Belgien

${ }^{2} \mathrm{HNO}-$ Praxis am Neckar, Heidelberg, Deutschland

3Universität Düsseldorf, Düsseldorf, Deutschland

${ }^{4}$ Charité Berlin, Berlin, Deutschland

Die TAV wurde 2008 eingeführt mit dem Ziel, für häufige Allergene (Gräser, Birke/Frühblüher, Milben und Bienen/Wespen) zukünftig nur noch Präparate mit belegter Wirksamkeit zuzulassen und am Patienten einzusetzen. Hier stellen wir den aktuellen Status hinsichtlich der Entwicklung der Präparate im Rahmen des TAV-Prozesses dar.

Neben dem aktuellen Zulassungsstand nach dem PaulEhrlich-Institut (PEI) wurden hierfür die Tabellen der aktuellen S2k-Leitlinie zur Übersicht der SIT-Produkte, wie auch zur Nachzulassung von TAV-Präparaten und die dort hinterlegten Studien über www.clinicaltrialsregister.eu ausgewertet.

Ergebnisse: Es ist deutlich, dass es bereits heute zugelassene Präparate unterschiedlicher Hersteller beider Applikationsformen für die 3 Hauptallergene (Gräser, Birke/Frühblüher, Milben) mit unterschiedlich starken Studienbelegen (Evidenzgrad) gibt. Allerdings hat sich bislang für kein Produkt im Nachzulassungsprozess der TAV die marktübliche Dosis als optimal herausgestellt. Für diese Produkte mussten die Dosierungen für die Phase III Studien um den Faktor 1,4-5,35 erhöht werden; hier wird es evtl. Neuzulassungen mit neuer Dosierung, neuen Produktnamen, aber zunächst ohne Kinderzulassung geben. Für weitere Produkte laufen entweder noch Dosisfindungsstudien (Phase II), oder es gibt keine Information zur Weiterentwicklung.
Schlussfolgerung: Bereits vor dem voraussichtlichen Abschluss der TAV im Jahre 2026 sind bereits heute Präparate mit Wirksamkeitsnachweis und Zulassung zu identifizieren, deren Einsatz im Sinne einer verantwortungsvollen und patientenorientierten ärztlichen Haltung, unter Wahrung der Rechte des Patienten (Patientenrechtegesetz 2013) und einem kosteneffektiven Umgang mit den finanziellen Mittel der Solidargemeinschaft (SGB V) unzweifelhaft der Vorzug zu geben ist. Die TAV leistet einen wertvollen Beitrag, der SIT, als einziger kausaler Therapieform bei Allergien, den Stellenwert eines Fertigarzneimittels zu verleihe

\section{P3.3}

Die SQ Frühblüher SLIT-Tablette reduziert

Rhinokonjunktivitissymptome und Medikamentenverbrauch während der drei Baumpollensaisons (Hasel-, Erle- und Birkenpollensaisons) Ergebnisse einer großen multizentrischen Phase-III-Studie

\section{U. Darsow ${ }^{1}$, D. Thrane ${ }^{2}$, S. Jacobsen ${ }^{2}$, H. Sørensen², H. Wolf ${ }^{3}$, T. Biedermann ${ }^{1}$}

${ }^{1}$ Klinik und Poliklinik für Dermatologie und Allergologie am Biederstein, TU München, München, Deutschland

${ }^{2}$ Global Pharmacovigilance \& Clinical Development, ALK A/S, Hørsholm, Dänemark

${ }^{3}$ Clinical Operations, ALK Abelló Arzneimittel GmbH, Hamburg, Deutschland

Hintergrund: Die SQ Frühblüher SLIT-Tablette (ALK, Dänemark) wird zur täglichen Einnahme zuhause für die Behandlung der mittelgradigen bis starken allergischen Rhinokonjunktivits durch Pollen der birkenhomologen Gruppe entwickelt. Hier werden die Ergebnisse einer Phase III-Studie berichtet.

Methoden: Die Studie TT-04 (EudraCT 2015-004821-15) war eine randomisierte, DBPC Phase III-Studie. 634 Patienten wurden 1:1 für die SQ Frühblüher-Tablette (Dosis 12 DU) oder Placebo randomisiert und für mindestens 16 Wochen vor Beginn der Baumpollensaison 2017 (Hasel, Erle, Birke) behandelt. Wirksamkeits-Endpunkte waren der tägliche Rhinitis/Konjunktivitis-Symptom(DSS)- und -Medikationsscore (DMS) sowie der kombinierte Gesamtscore als Summe der beiden Scores (Total Combined Score, TCS) währen der Birkenpollen(BPS)und der Baumpollensaison (TPS). Primärer Endpunkt war der mittlere TCS währen der BPS. Haupt-Sicherheitsendpunkte waren Unerwünschte Ereignisse (UEs).

Ergebnisse: TCS, DSS und DMS in der BPS und TPS waren mit der SQ Frühblüher SLIT-Tablette alle signifikant gegenüber Placebo reduziert. Der mittlere TCS in der BPS (primärer Endpunkt) war mit der SQ Frühblüher SLIT-Tablette um 39,6\% vs. Placebo signifikant reduziert und in der TPS um $36,5 \%$. Der mittlere DSS in der BPS war um $36,8 \%$ und in der TPS um $32,7 \%$ geringer vs. Placebo (alle $p<0.0001$ ). DSS und DMS trugen annähernd gleich zum beobachteten Behandlungseffekt während der BPS und TPS bei. Der TCS während der Haselund Erle-Saison wurde post-hoc analysiert und war um 29,7 \% geringer vs. Placebo $(p=0,0015)$. Die Behandlung wurde gut vertragen. Häufigste behandlungsbezogene UEs waren leichte bis mittelgradige Lokalreaktionen in Mund und Rachen am 1. und 2. Behandlungstag. Es wurden keine anaphylaktischen Reaktionen beobachtet.

Schlussfolgerung: Die TT-04-Studie hat ein positives Nutzen-Risiko-Verhältnis mit einem klinisch relevanten Behand- 
lungseffekt der SQ Frühblüher SLIT-Tablette während der gesamten TPS gezeigt.

\section{P3.4}

\section{Ergebnisse der Rush-Aufdosierung} mit HAL Pollenallergoidpräparaten in der breiten Anwendung bestätigen die gute Verträglichkeit in Übereinstimmung mit den Phase IV-Studien

\section{A. Distler ${ }^{1}$, H. van Schijndel ${ }^{2}$, D. $\mathbf{Y u}^{2}$, D. Pappelendam², N. van Os ${ }^{1}$, M. Leineweber ${ }^{1}$}

${ }^{1} \mathrm{HAL}$ Allergie $\mathrm{GmbH}$, Düsseldorf, Deutschland ${ }^{2}$ HAL Allergy B. V., Leiden, Niederlande

Hintergrund: Ergebnisse zur Rush-Aufdosierung mit drei subkutanen Injektionen in zwei Wochen bei Erwachsenen aus zwei Phase IV-Studien mit Gräser- (Pfaar et al. Int Arch Allergy Immunol 2013; 160:420-424) bzw. Birkenpollen (Buczylko et al. Int Arch Allergy Immunol 2017; 172:183-168) wurden mit den Ergebnissen einer nicht-interventionellen Studien (NIS) verglichen (Distler et al. EAACI 2018 Abstract 1213).

Methode: In den beiden Phase IV-Studien wurde die zuvor mit dem Paul-Ehrlich-Institut abgestimmte statistisch notwendige Anzahl an Patienten (P) mit dem Rush-Schema therapiert. In der NIS konnte eine deutlich größere Zahl an P eingeschlossen werden. Im direkten Vergleich der Studiendaten sollte nun überprüft werden, ob und wie sich die Ergebnisse der NIS in Bezug auf Anzahl P, die die Höchstdosis (HD) von 0,5 ml nach drei Injektionen mit PURETHAL ${ }^{\circ}$ (HAL Allergy B. V., Leiden, Niederlande) erreichten, und in den Verträglichkeitsdaten unterschieden.

Ergebnisse: In der Phase IV-Studie mit Gräsern ( $n=146$; davon 69 Rush) erreichten 92,8\% mit dem Rush-Schema die HD von $0,5 \mathrm{ml}$. In der NIS waren es $97,5 \%(n=435)$.

Bei Birke waren es in der Phase IV-Studie 98,4\% $(n=123$; davon 62 Rush). In der NIS konnten sogar 98,8\% $(n=449)$ die HD erreichen.

Verträglichkeitsdaten - Gräser: Phase IV: lokale Sofortreaktion(LSoR)/Spätreaktion (LSpR) $<5 \mathrm{~cm} \quad 27,5 \%$, $\geq 5 \mathrm{~cm} \mathrm{29 \% *;} \mathrm{systemische} \mathrm{Sofortreaktion} \mathrm{(SSoR)/Spätreaktion}$ (SSpR) 10,1\%*

NIS: LSoR 14,3 \%; LSpR 31,1 \%; SSoR 1,2 \%; SSpR 6,3 \%

Verträglichkeitsdaten - Birke: Phase IV: LSoR $\leq 5 \mathrm{~cm}$ : 14-19 \%; LSpR $\leq 5 \mathrm{~cm} \mathrm{24,4 \% ,} \mathrm{<12} \mathrm{cm} \mathrm{16,3 \% ;} \mathrm{SSoR} \mathrm{keine} \mathrm{Anga-}$ ben; SSpR 6,4 \%

NIS: LSoR 13,7 \%; LSpR 33,8 \%; oR 1,6 \%; SSpR 9,4 \%

Schwere systemische Reaktionen (Grad III und IV) traten nicht auf.

Zusammenfassung: Die Ergebnisse der NIS stimmen in Bezug auf Erreichen der HD und Verträglichkeit mit den Ergebnissen der Phase IV-Studien überein. Die Rush-Aufdosierung hat sich auch in der täglichen Routine mit einem sehr guten Sicherheitsprofil bewährt.

${ }^{*}$ Keine Angaben, ob Sofort- oder Spätreaktion

\section{P3.5}

\section{Einfluss von Vitamin D auf die subkutane Gräserpollen-spezifische Immuntherapie - Daten einer kontrollierten Pilotstudie}

\section{G. Heine ${ }^{1}$, W. Francuzik ${ }^{1}$, S. Dölle ${ }^{1}$, G. Drozdenko', N. Schumacher ${ }^{1}$, P. Bacher ${ }^{1}$, A. Scheffold ${ }^{1}$, A. Radbruch ${ }^{2}$, M. Worm}

${ }^{1}$ Charité - Universitätsmedizin Berlin, Berlin, Deutschland ${ }^{2}$ Deutsches Rheuma-Forschungszentrum Berlin, LeibnizGemeinschaft, Berlin, Deutschland

Vitamin D moduliert die allergische Immunantwort. Eigene Vorarbeiten belegen, dass aktivierte B-Lymphozyten den aktiven Vitamin D-Metabolit Calcitriol (1,25-Dihydroxyvitamin D3) aus dem Speichermetaboliten 25-Hydroxyvitamin D3 (25OHD) herstellen können. Ferner wird durch Calcitriol die IgE-Produktion gehemmt und IL-10 gefördert. Präklinische Daten deuten an, dass durch Vitamin D die Allergen-spezifische Immuntherapie verstärkt wird, gemessen an einer verminderten IgE-Antwort und allergischen Atemwegsinflammation.

In der vorgestellten Untersuchung wurden Patienten mit Gräserpollen-induzierter allergischer Rhinokonjunktivitis \pm Bronchialasthma mit in 3 aufeinander folgenden Jahren präsaisonal mit spezifischer Immuntherapie behandelt. Gleichzeitig wurde doppelblind, randomisiert 5333 I. U. Vitamin D oder Plazebo oral eingenommen (ProGIT; NCT01466465). Es wurden spezifische Hauttestungen (titrierter i.c., konjunktivale Provokation, Pricktest), klinische Scores und immunologische Parameter erfasst. Mittels der innovativen ARTE-Technologie (Antigen-Responsive-T cell-Enrichment) wurden spezifische T-Helfer und regulatorische T-Zellen synchron unter Immuntherapie bestimmt.

Die Daten zeigen eine effektive Vitamin D Supplementation bei sehr guter Verträglichkeit und Sicherheit (Unterschied der 25OHD-Konzentration zur Placebogruppe $=p<0,001)$. Bei titrierter Intrakutantestung und Pricktestung zeigt sich durch die spezifische Immuntherapie eine verminderte Reaktivität, die in der Vitamin D-Gruppe früher auftritt. Die initiale humorale spezifische IgE-Antwort ist in der Vitamin D-Gruppe vermindert gegenüber Placebo und wird über die Gesamttherapiedauer abgeschwächt, bei vergleichbarer Induktion von IgG4. Die Analyse von spezifischen T-Helfer und regulatorischer T-Zellen sowie B-Zellantworten identifiziert ein charakteristisches Wirkmuster der Verumgruppe und bietet weitere Ansätze zur Identifikation der Wirkmechanismen der spezifischen Immuntherapie.

\section{P3.6}

\section{Sicherheit und Verträglichkeit einer verkürzten Aufdosierung einer SCIT mit einem Gräserpollenallergoid}

\section{Kopp', L. Klimek², K. Plückhahn³ ${ }^{3}$ F. Kreimendahl³, G. Fox ${ }^{3}$}

${ }^{1}$ Sektion für Pädiatrische Pneumologie und Allergologie, Kinder- und Jugendmedizin, Universitätsklinik Lübeck, Airway Research Center North, (Deutsches Zentrum für Lungenforschung, Lübeck, Deutschland ${ }^{2}$ Zentrum für Rhinologie und Allergologie, Wiesbaden, Deutschland

${ }^{3}$ Allergopharma GmbH \& Co. KG, Reinbek, Deutschland 
Hintergrund: Die subkutane Allergen-Immuntherapie (SCIT) mit einem hochdosiertem Gräserpollen-Allergoid ist eine wirksame und verträgliche Behandlungsoption, die seit 1992 in Deutschland zugelassen ist. Die Aufdosierung erfolgt standardmäßig mit 4 oder 7 Injektionen bei Verwendung von 2 Stärken $(A+B)$. In dieser Studie wurde untersucht, ob eine Verkürzung der Aufdosierung auf 3 Injektionen ohne Verwendung der geringer konzentrierten Stärke A sicher und verträglich ist.

Methode: In diese randomisierte, multizentrische Phase II-Vergleichsstudie (EudraCT-Nr. 2017-000754-19) wurden 86 Patienten (18-65 Jahre) mit einer Gräserpollen-allergischen Rhinokonjunktivitis \pm Asthma eingeschlossen. Sicherheit und Verträglichkeit des Standard-Aufdosierungsschemas mit 7 Injektionen (4 Injektionen Stärke A, 3 Injektionen Stärke B) des 6-Gräserpollenallergoids wurden mit der verkürzten Aufdosierung aus 3 Injektionen der Stärke B verglichen. Unerwünschte lokale und systemische Arzneimittelwirkungen (ADRs) wurden nach dem WAO-Grading klassifiziert. Patienten und Prüfärzte bewerteten unabhängig voneinander die Verträglichkeit der SCIT anhand einer 5-Punkte-Likert-Skala.

Ergebnisse: Je 43 Patienten wurden in beiden Gruppe randomisiert. Insgesamt gab es keine signifikanten Unterschiede in der Anzahl der ADRs [120 ADRs bei 27 Patienten (3 Injektionen), 125 ADRs bei 24 Patienten (7 Injektionen)]. Dabei waren mehr als $85 \%$ aller ADRs von milder Intensität und es traten keine systemischen Reaktionen mit WAO Grad > 2 auf. Die Mehrzahl aller ADRs trat innerhalb von 2 Stunden nach der Injektion auf.

Schlussfolgerung: Die Aufdosierung mit nur 3 Injektionen der Stärke B ist vergleichbar sicher und verträglich wie das Standarddosierschema mit 7 Injektionen bei erwachsenen Gräserpollenallergikern. Sie hat den Vorteil, dass die Verwechslung der Stärken A und B bei der Anwendung ausgeschlossen ist. Für die Patienten kann sich die Zeit der Aufdosierung von 6 auf 2 Wochen reduzieren.

\section{P3.7}

Ein verkürztes Aufdosierungsschema

für sublinguale Immuntherapie

gegen Baumpollen-induzierter Allergie ist

gut verträglich und führt zu dosisabhängigen

klinischen Effekten während der Pollensaison

\section{R. Mösges ${ }^{1}$, N. Breitrück', S. Allekotte' ${ }^{2}$, K. Shah- Hosseini', P. Zieglmayer', K. Birkholz ${ }^{4}$, M. Hess' ${ }^{2}$, M. Kmenta ${ }^{5}$, K. Bastl ${ }^{5}$, U. Berger ${ }^{5}$, M. Kramer ${ }^{4}$, S. Guethoff 4}

${ }^{1}$ Institut für Medizinische Statistik und Bioinformatik (IMSB), Medizinische Fakultät, Universität zu Köln, Köln, Deutschland ${ }^{2} \mathrm{CRI}$-Clinical Research International Ltd., Köln, Deutschland ${ }^{3}$ Allergiezentrum Wien West, Wien, Österreich ${ }^{4}$ Bencard Allergie GmbH, München, Deutschland ${ }^{5}$ Klinische Abteilung für Allgemeine Hals-, Nasen- und Ohrenkrankheiten, MedUni Wien, Wien, Österreich

Einleitung: Um die Adhärenz von Patienten zu einer spezifischen Immuntherapie (SIT) zu verbessern, ist eine optimierte und unkomplizierte Aufdosierung wünschenswert. Ziel dieser Studie war es, ein verkürztes Aufdosierungsschema einer sublingualen SIT (SLIT) zur Therapie einer Baumpollen-Allergie, welche bis auf die Initialdosis daheim durchgeführt wird, mit zwei bisherigen Aufdosierungsschemata zu vergleichen und den Therapieeffekt in der ersten Pollensaison zu analysieren.
Methoden: In dieser offenen, prospektiven, nicht-interventionellen Studie wurde die Verträglichkeit drei verschiedener Aufdosierungsschemata sowie die klinische Verbesserung (Symptome, Medikation) nach dem ersten Therapiejahr untersucht.

Ergebnisse: An 33 Zentren in Deutschland und Österreich wurden 164 Patienten im Jahr 2016/2017 dokumentiert. Die Therapie war unabhängig vom gewählten Aufdosierungsschema gut verträglich. Lokalreaktionen hatten meistens eine milde Intensität und es gab keine schwerwiegenden Ereignisse. Es wurde eine Reduktion der Symptom-Scores gegenüber dem Vorjahr 2016 beobachtet. Der Prozentsatz an Patienten mit einer persitierenden, moderat-schweren Rhinitis nach ARIAKlassifikation von $79 \%$ reduzierte sich nach Therapie auf $19 \%$. Insgesamt erreichten $62 \%$ der Patienten eine Symptomkontrolle der Rhinitis und $34 \%$ benötigten nach einem Therapiejahr keine symptomatische Medikation mehr. Die Reduktion des Rhinokonjunktivitis-Scores war bei prä-saisonal gestarteter Therapie um $34 \%$ höher als bei Patienten der Kontrollgruppe mit co-saisonalem Therapiestart. Hingegen wies der allgemeine Symptomindex aller Personen in Deutschland und Wien, die ihre Symptome in einer Pollen App eintrugen, für die Baumpollensaison 2017 nur geringfügig schwächere Symptome im Vergleich zu 2016 auf.

Zusammenfassung: Die SLIT war unabhängig vom gewählten Aufdosierungsschema gut verträglich und führte bereits im ersten Therapiejahr zu einer Verbesserung der Symptome und Reduktion der Bedarfsmedikation.

\section{P3.8}

Korrelation der Rhinokonjunktivitis-Symptomatik bei Gräserpollenallergikern in der Expositionskammer und im Feld

\section{K. Plückhahn, S. Mußler, F. Kreimendahl, C. Prächter, G. Fox, C. Willers}

Allergopharma GmbH \& Co. KG, Reinbek, Deutschland

Hintergrund: Expositionskammern bieten gegenüber der natürlichen Exposition den Vorteil, dass die Patienten Allergenen in standardisierter Menge, Qualität und Dauer ausgesetzt werden. Bisher gibt es nur wenige Studien, die die Korrelation der Symptomatik in der Kammer mit dem Symptom-Medikationsscore (SMS) in Feldstudien vergleichen. Entsprechend werden Kammerstudien derzeit für pivotale Phase III SIT-Studien von den Zulassungsbehörden nicht akzeptiert.

Methode: In die Baselinephase einer Phase II-Studie in Kanada wurden 70 Graspollenallergiker (18-65 Jahre) und 21 Nichtallergiker eingeschlossen. Diese wurden vor und nach der Gräserpollensaison an je 3 aufeinanderfolgenden Tagen für je 6 Stunden in der Kammer (Inflamax, Kanada) Gräserpollen $\left(3500 \pm 500 \mathrm{Körner} / \mathrm{m}^{3}\right)$ ausgesetzt. Teilnehmer bewerteten ihre Augen- und Nasensymptome auf einer 4-stufigen Skala, so dass Gesamt(TSS)-, Nasen(TNSS)- und Augen(TOSS)-Symptomscores berechnet wurden. Während der Gräserpollensaison dokumentierten sie für die Ermittlung eines SMS täglich ihre allergischen Symptome und den Medikationsgebrauch. Im Falle von klinisch relevanter Korrelation sollte eine SCIT mit einem Gräserallergoid folgen.

Resultate: Die Interimsanalyse der Baselinephase ergab für die Graspollenallergiker signifikante Korrelationen $(p<0,05)$ zwischen dem AUC des Rhinokonjunktivitis-SMS im Feld und des TSS in der Kammer. Der Korrelationskoeffizient nach Pear- 
son zeigte eine schwache Korrelation von 0,239 bis 0,319 für die AUC des SMS. Nichtallergiker berichteten keine relevanten allergischen Symptome. Aufgrund der Stärke der Korrelation wurde die Studie abgebrochen.

Schlussfolgerung: Bei Gräserpollenallergikern war die gewählte Methodik der Korrelation zwischen dem SMS im Feld und dem TNSS in der Kammer nur schwach bis mäßig ausgeprägt. Auch eine Kammerexposition über 3 aufeinanderfolgende Tage führte zu keiner besseren Korrelation. Daher gilt es, neue, innovative Designs der Korrelationsanalyse zu reflektieren.

\section{P3.9}

Wirksamkeit und Sicherheit einer SQ Hausstaubmilben(HSM)-Tablette zur sublingualen Immuntherapie in japanischen Kindern mit perennialer allergischer Rhinitis: Eine randomisierte, doppelblinde, placebokontrollierte Phase III-Studie

\section{H. Wolf', K. Okubo ${ }^{2}$, H. Nolte ${ }^{3}$, Y. Okamoto ${ }^{4}$, R. Azuma ${ }^{5}$, K. Natsui ${ }^{5}$, K. Matsuyama ${ }^{6}$}

${ }^{1}$ Clinical Operations, ALK-Abelló Arzneimittel GmbH, Hamburg, Deutschland

${ }^{2}$ Nippon Medical School, Tokio, Japan

${ }^{3} \mathrm{ALK}$, Bedminster, USA

${ }^{4}$ Chiba University, Chiba, Japan

${ }^{5}$ Torii Pharmaceutical, Tokio, Japan

${ }^{6}$ Yamanashi University, Tokio, Japan

Hintergrund: Die Wirksamkeit einer lyophilisierten SQ Hausstaubmilben(HSM)-SLIT-Tablette (T-203, Torii, Japan/ALK, Dänemark) bei der Behandlung der perennialen respiratorischen Allergie gegen HSM wurde für Patienten $\geq 12$ Jahre in klinischen Studien in Japan, Nordamerika und Europa nachgewiesen. Diese Studie untersucht die Wirksamkeit und Sicherheit bei japanischen Kindern und Jugendlichen (5-17 Jahre) mit mittelgradig bis starker allergischer Rhinitis (AR) gegen HSM.

Methoden: In der randomisierten, placebokontrollierten Doppelblindstudie wurden 458 japanische Kinder mit einem AR Symptomscore $\geq 7$ von max. 12 an mindestens 7 Tagen während einer 14-tägigen Run-in-Periode ohne symptomatische Behandlung entweder für eine 1-jährige Behandlung mit der SQ HSM SLIT-Tablette (10.000 Japanese Allergy Units, JAU) oder Placebo 1:1 randomisiert. Primärer Endpunkt war der kombinierte Rhinitis-Gesamtscore (Total Combined Rhinitis Score, TCRS), der die AR Symptom- und Medikationsscores während der letzten 8 Wochen der Behandlung zusammenfasst.

Ergebnisse: Der TCRS war um $23 \%$ (95\% CI [14\%;31\%]) unter aktiver Therapie vs. Placebo reduziert. Prädefinierte, stratifizierte Analysen ergaben eine ähnliche Reduktion des TCRS für Kinder von 5-11 Jahren (-21\%, $95 \%$ CI [8\%;32\%]) und für Jugendliche von $12-17$ Jahren $(-26 \%$, $95 \%$ CI [11 \%;38 \%]). Es wurden keine anaphylaktischen Reaktionen oder schwerwiegende Unerwünschte Arzneimittel-Wirkungen (UAW) berichtet. Häufigste Reaktionen waren leichte Lokalreaktionen an der Applikationsstelle. Die Behandlung wurde von Kindern und Jugendlichen gleich gut vertragen.

Schlussfolgerung: Die Studie hat die klinisch relevante Wirksamkeit und ein akzeptables Sicherheitsprofil bei japanischen Kindern und Jugendlichen mit mittelgradig bis starker HSM-AR ergeben. Die Ergebnisse sind konsistent mit Daten für japanische Kinder, Jugendliche und Erwachsene.

\section{P3.10}

Durch die SQ Frühblüher SLIT-Tablette

induzierte IgG4-Kreuzrektivität erweitert

den immunmodulierenden Effekt auf

die gesamte birkenhomologe Gruppe

\section{P. Adler Würtzen, H. Ipsen, J. Damkjær, P. Grønager, M. Steffensen, P. Andersen}

ALK A/S, Hørsholm, Dänemark

Hintergrund: In Europa und Nordamerika verursachen Allergene der birkenhomologen Gruppe allergische Rhinokonjunktivitis, darunter Birke, Hasel, Erle und Hainbuche, die alle durch Bet v1-homologe Allergene charakterisiert sind. Die hohe Sequenz-Identität der Bet v 1-Homologen führt zu weitgehender Kreuzreaktivität. Studienziel war es, zu untersuchen, ob die SQ Frühblüher SLIT-Tablette (Birkenallergenextrakt) mit allen Spezies in der birken-homologen Gruppe kreuzreagierende immunologische Antworten induziert und damit der zuvor bestimmte signifikante klinische Effekt für Birke und Eiche auf die gesamte birken-homologe Gruppe ausgeweitet werden kann.

Methoden: In Blutproben Erwachsener mit mittelgradiger bis starker Rhinokonjunktivitis gegen Birke wurden IgE vor und IgG4 nach 24-wöchiger Behandlung mit der SQ Frühblüher SLIT-Tablette (Studie TT-03, NCT02481856), spezifisch für Birke, Eiche, Hasel, Erle und Hainbuche bestimmt (ImmunoCAP) und die immunologische Kreuzreaktivität mit Korrelationsanalysen und Inhibitionsassays untersucht.

Ergebnisse: Die Korrelation der IgE-Reaktivität (vor Behandlung) zwischen Birke und Erle, Hasel, Hainbuche und Eiche war am höchsten zwischen Birke und Erle $(r=0,98)$ und am niedrigsten zwischen Birke und Eiche $(r=0,83)$. Die behandlungs-induzierte IgG4-Reaktivität war zudem hoch korreliert zwischen Birke und Erle, Hasel, Hainbuche, Buche und Eiche, mit der höchsten Korrelation zwischen Birke und Erle $(\mathrm{r}=0,95)$ und der niedrigsten zwischen Birke und Eiche $(r=0,78)$. In der Mehrzahl der Patienten wurde die erle-, hasel und eiche-spezifische IgE- und IgG4-Reaktivität um mehr als $80 \%$ durch Birkenallergenextrakt inhibiert.

Schlussfolgerung: Die Daten zeigen eine hohe Kreuzreaktivität zwischen birke-spezifischem IgE und IgG4 und Allergenen der birkenhomologen Gruppe, was daraufhin deutet, dass der zuvor gemessene signifikante klinische Effekt für Birken- und Eichenexposition auf die gesamte birkenhomologe Gruppe ausgeweitet werden kann. 
P3.11

Results of a phase II allergen immunotherapy study to determine the optimally effective and safe dose of subcutaneously administered tyrosine adsorbed modified grass allergen + MPL

S. Zielen', P. Kuna², W. Arberer', S. Lassmann 4 , A. Wade ${ }^{5}$, K. Klühr ${ }^{6}$, J. Raab ${ }^{6}$, D. Lee ${ }^{6}$, R. Ballard ${ }^{5}$, C. Jones ${ }^{5}$, K. Gunawardena ${ }^{5}$, T. Higenbottam ${ }^{5}$, M. Skinner

${ }^{1}$ University Hospital Frankfurt, Frankfurt, Germany ${ }^{2}$ Barlicki University Hospital, Medical University of Lodz, Lodz, Poland

${ }^{3}$ Department of Dermatology, Medical University of Graz, Graz, Austria

${ }^{4}$ Practice Dr.med. Sabine Lassmann - Specialist in Otolaryngology, Saalfeld, Germany

${ }^{5}$ Allergy Therapeutics, Worthing, United Kingdom

${ }^{6}$ Bencard Allergie GmbH, Munich, Germany

Introduction: There is increasing evidence that effectiveness of allergy immunotherapy to control ARC symptoms is related to the cumulative dose administered. Previous studies showed a 5.5 fold increase in cumulative dose to achieve 50\% increase in efficacy, with a relative reduction in total symptom score (TSS) of $32.3 \%$ compared to placebo and no safety signals of concern. The shape of the dose response curve was curvilinear, where this high dose almost reached plateau. We report on a Phase II dose-finding study [2017-000333-31] of a modified grass allergen subcutaneous immunotherapy (SCIT) product with modified allergen tyrosine adsorbate (MATA) and monophosphoryl lipid A (MPL) adjuvants for the treatment of allergic rhinoconjunctivitis (ARC), which included up to 7 fold higher doses.

Methods: This was a European multi-center, randomized, double-blind, placebo-controlled, parallel-group study in adult patients (pts) with moderate to severe seasonal ARC with/without mild asthma. The primary outcome was the post-treatment TSS following conjunctival provocation test (CPT). Different cumulative dose regimens $(5100,14.400,27.600,35.600 \mathrm{SU}$, or placebo) were applied over 6 weekly injections to establish the shape of the dose response to support dose selection for Phase 3.

Results: 546 pts were screened and pts were randomized. The post-treatment TSS showed a statistically significant doseresponse relationship and IgG4 increase was consistent with the dose response. All dosing regimens were safe and well tolerated. The currently marketed product ( $5100 \mathrm{SU})$ showed a significant improvement compared to placebo $(p<0.01)$. The adherence to the short treatment course was $>95 \%$.

Conclusions: This Phase 2 study was conducted to establish the dose response of a grass MATA MPL SCIT product, using CPT to measure the effect of a wide range of cumulative dose regimens. The results are an important milestone in the development of an efficacious and safe state-of-the-art gras SCIT.
4. Specific immunotherapy - basics and laboratory techniques

\section{P4.1}

\section{Aufklärung der Allergenzusammensetzung Allergoid-basierter Pollen-Allergenprodukte mittels Massenspektrometrie}

\section{S. Augustin', C. Scheibe', T. Eichhorn², F. Fischer ${ }^{2}$, C. Willers ${ }^{1}$}

${ }^{1}$ Allergopharma GmbH \& Co. KG, Reinbek, Deutschland ${ }^{2}$ Merck KGaA, Darmstadt, Deutschland

Hintergrund: Eine hohe Qualität von Allergenprodukten ist Voraussetzung für die erfolgreiche Diagnose und Therapie allergischer Erkrankungen. Allerdings variieren pharmazeutische Allergenpräparationen bezüglich des Vorhandenseins enthaltener Major- und Minorallergene. Während der Allergennachweis in nativen Allergenextrakten meist durch tierische Antikörper erfolgt, sind diese für chemisch-modifizierte Allergene (Allergoide) ungeeignet. Massenspektrometrie (MS) stellt eine Alternative für den Allergennachweis dar. In der vorliegenden Studie wurde die Allergenzusammensetzung Allergoid-basierter therapeutischer Allergenpräparationen durch MS analysiert.

Methode: Allergoid-basierte Allergenpräparationen (Aluminium-Adsorbate; jeweils 3 Chargen) der Allergenquellen Birke, Beifuß und einer 6-Gräser-Mischung wurden mittels MS untersucht. Nach Desorption der Allergoide wurden diese durch tryptischen Verdau in Peptide gespalten und die Allergene via Tandem-Massenspektrometrie (LC-MS/MS) identifiziert.

Ergebnisse: Neben den Majorallergenen der Allergenquellen Birke (Bet v 1), Beifuß (Art v 1) und Gräser (Gruppe 1 \& 5) konnten in den jeweiligen Allergenpräparationen durch MS zusätzlich alle weiteren in der Allergendatenbank IUIS aufgeführten Allergene der untersuchten Spezies nachgewiesen werden. Dabei handelte es sich bei den Allergenpräparationen der Birke um die Allergene Bet v 2, Bet v 3, Bet v 4, Bet v 6, Bet v 7 und Bet v 8, bei denen des Beifußes um die Allergene Art v 2, Art v 3, Art v 4, Art v 5 und Art v 6 und bei denen der 6-GräserMischung um die Allergene der Gruppen 2, 3, 4, 6, 7, 11, 12 und 13.

Schlussfolgerung: MS ist eine geeignete Methode, um die Allergenzusammensetzung Aluminium-adsorbierter Allergoide aufzuklären. Es konnten alle Major- und Minorallergene der jeweiligen Spezies in jeweils 3 Chargen nachgewiesen werden. Diese Ergebnisse belegen die Chargenvergleichbarkeit und die hohe Qualität der untersuchten Allergenpräparationen. 
P4.2

Charakterisierung der primären und sekundären Adsorbat-Partikel in Produkten für die subkutane Immuntherapie

\section{A. Cossé1, C. Scheibe ${ }^{1}$, M. Wald', L. Pump', L. Petzinger ${ }^{2}$, H. Weiser ${ }^{2}$, D. Wandschneider ${ }^{2}$, C. Willers ${ }^{1}$, S. Augustin ${ }^{1}$}

${ }^{1}$ Allergopharma GmbH \& Co. KG, Reinbek, Deutschland ${ }^{2}$ Merck KGaA, Darmstadt, Deutschland

Hintergrund: Aluminium-Verbindungen werden häufig als Trägermaterial für Wirkstoffe in Produkten zur subkutanen allergenspezifischen Immuntherapie (SCIT) eingesetzt. Bei der Herstellung werden die Allergene oder Allergoide (chemisch-modifizierte Allergene) meist an Aluminiumhydroxid adsorbiert. Die resultierende parenterale Suspension enthält sichtbare Adsorbat-Partikel. Die Morphologie und die Partikelgrößenverteilung von Aluminiumhydroxid-Adsorbaten wurden mit Hilfe der Laserdiffraktometrie und der Rasterelektronenmikroskopie (REM) untersucht.

Methoden: Es wurden zwanzig Chargen eines Gräserallergoid-Produktes (Allergopharma) analysiert. Die Partikelgrößenverteilung der sichtbaren Partikel wurde mit einem Mastersizer 2000 Laserdiffraktometer mit Hydro SV $2000 \mu$ P Messzelle (Malvern Instruments Ltd) bestimmt und als Volumen-basiertes Verteilungsprofil dargestellt. Zudem wurde die Größenverteilung durch Berechnung der Werte für den Median $\left(d_{v} 0.5\right)$ und die Verteilungsbreite $\left(\mathrm{d}_{\mathrm{v}} 0.1, \mathrm{~d}_{\mathrm{v}} 0.9\right)$ analysiert. Die REMAufnahmen wurden mit einem Supra 35/LEO 1530 Mikroskop (Zeiss) erzeugt.

Ergebnisse: Die Größenverteilung der sichtbaren Partikel war in den untersuchten Chargen vergleichbar $\left(\mathrm{d}_{\mathrm{v}}(0.5)\right.$ : 20-28 $\mu \mathrm{m}$ (Mittelwert: $23 \mu \mathrm{m}$ ), $\mathrm{d}_{\mathrm{v}}(0.1): 10-13 \mu \mathrm{m}$ (Mittelwert: $11 \mu \mathrm{m})$ und $d_{v}(0.9)$ : 36-56 $\mu \mathrm{m}$ (Mittelwert: $44 \mu \mathrm{m}$ )). Die REMBilder zeigten, dass die sichtbaren Partikel aus agglomerierten Primärpartikeln mit einem Durchmesser von 16-20 nm bestanden.

Schlussfolgerung: Durch die hochaufgelöste Mikroskopie wurden die Struktur der in der Suspension sichtbaren Partikel aufgedeckt und die zugrundeliegenden Primärpartikel identifiziert. Die sichtbaren Partikel können somit als Sekundärpartikel eingestuft werden. Die äußerst geringe Chargen-Variabilität des getesteten Allergoid-Produktes bezüglich der Partikelgrößenverteilung zeugt von einem reproduzierbaren Herstellungsprozess und einer gleichbleibenden Produktqualität.

\section{P4.4}

\section{Herstellung der rekombinanten fungalen}

Serinproteasen Pen ch 13 und Pen ch 18 und Charakterisierung ihrer IgE-Bindungsfähigkeit

\section{S. Kespohl, J. Gleichenhagen, S. Maryska, T. Brüning, G. Johnen, M. Raulf}

Institut für Prävention und Arbeitsmedizin der Deutschen Gesetzlichen Unfallversicherung, Institut der Ruhr-Universität Bochum, IPA, Bochum, Deutschland

Die prominenteste Proteinfamilie fungaler Allergene sind Serinproteasen, für die Sensibilisierungsraten von 15 bis $\mathrm{zu} \quad 80 \%$ unter Schimmelpilzsensibilisierten beschrieben wurden. Insbesondere bei Patienten mit schweren respiratorischen Symptomen wie Asthma wurden hohe sIgESensibilisierungsraten gegen eine alkalische Serinprotease aus Penicillium chrysogenum (Pen ch 13) und eine vakuoläre Serinprotease (Pen ch 18) gemessen. Obwohl es schon Daten zu Serinproteasen als relevante Schimmelpilzallergene gibt, sind sie bisher nicht für die Diagnostik verfügbar.

Basierend auf den publizierten Aminosäuresequenzen für Pen ch 13 (UniProtID: Q9URR2) und Pen ch 18 (UniProtID: P49768.1) wurden entsprechende Gene inklusive Histidin-tag konstruiert und synthetisiert Die Klonierung sowie rekombinante Proteinexpression erfolgte in E. coli Bakterien. Die rekombinanten Allergene rPen ch 13 und rPen ch 18 wurden isoliert, nach Biotinylierung an ImmunoCAPs gekoppelt und die spezifische IgE(sIgE)-Bindung getestet.

Die Allergene rPen ch 13 und rPen ch 18 zeigten in der Coomassie-SDS-PAGE einen hohen Reinheitsgrad sowie die entsprechenden Molekulargewichte von $32 \mathrm{kDa}$ (rPen ch 13) und 38-40 kDa (rPen ch 18). Die sIgE-Bindung wurde in 57 Seren von Patienten mit sIgE-Sensibilisierung auf Penicillium chrysogenum getestet. Spezifisches IgE $\geq 0,35 \mathrm{kU} / \mathrm{L}$ gegen $\mathrm{rPen} \mathrm{ch}$ 13 wurde in 6 Seren gemessen, 4 dieser Seren hatten auch sIgE gegen rPen ch 18. Die sIgE-Konzentration gegen rPen ch 13 war in allen Fällen höher, als die gegen rPen ch 18. Eine isolierte Sensibilisierung gegen rPen ch 18 konnte nicht detektiert werden. Alle Patienten mit sIgE gegen rPen ch 13 bzw. rPen ch 18 hatten asthmatische Beschwerden bzw. ABPA/Mykose basierte Dyspnoe.

Die rekombinanten Einzelallergene rPen ch 13 und rPen ch 18 wurden erfolgreich hergestellt. Weitere Studien zur Aufklärung einer möglichen Bedeutung als Markerallergene für klinisch-relevante Beschwerden, sowie Untersuchungen zur Kreuzreaktivität werden folgen.

\section{P4.5}

Vergleich von drei Testsystemen zur Bestimmung allergenspezifischer IgE-Antikörper

\section{B. Laffert, K. Bauermeister, A. Kappes, K. Tonn, C. Willers, S. Augustin}

Allergopharma GmbH \& Co. KG, Reinbek, Deutschland

Hintergrund: Die quantitative Bestimmung spezifischer IgE-Antikörper ist für eine sichere Allergie-Diagnose essentiell. Für die Bestimmung der Konzentration allergenspezifischer IgE-Antikörper sind zahlreiche Tests erhältlich. Die Ergebnisse dieser Methoden sind oft nicht vergleichbar. Es wurde z. B. über starke Unterschiede bei Tests für die Allergene von Bienengift, Wespengift und Birkenpollen berichtet.

Methoden: In der vorliegenden Studie wurden die Testsysteme von 3 Herstellern miteinander verglichen. Hierfür wurden die Konzentrationen allergenspezifischer IgE-Antikörper gegen 29 verschiedene Allergenextrakte (von Milben, Tierhaaren, Nüssen, Pilzen, Gräser-, Baum- und Kräuterpollen) in drei humanen Allergiker-Seren bestimmt.

Ergebnisse: Bei Hersteller 1 lagen $45 \%$ der Messwerte unterhalb von $0,35 \mathrm{kU} / \mathrm{l}$. Bei Hersteller 2 waren dies $16 \%$, und bei Hersteller 3 lediglich $10 \%$. Die Sensitivität der Testsysteme der Hersteller kann man aufsteigend folgendermaßen ordnen: Hersteller $1<$ Hersteller $2<$ Hersteller 3. Das Testsystem von Hersteller 3 hatte den kleinsten Variationskoeffizienten. Bei den Testsystemen der Hersteller 1 und 2 war der Variationskoeffizient vergleichbar und deutlich höher als bei Hersteller 3. 
Schlussfolgerung: Lediglich das Testsystem von Hersteller 3 erscheint für den Nachweis von Sensibilisierungen in Humanseren geeignet. Aufgrund der geringen Sensitivität von Testsystem 1 würden beim Einsatz dieses Testsystems viele Sensibilisierungen unerkannt bleiben. Das Testsystem von Hersteller 2 ist zwar noch ausreichend sensitiv, aber die Messergebnisse sind aufgrund des hohen Variationskoeffizienten nicht aussagekräftig. Die Ergebnisse der vorliegenden Studie zeigen die Notwendigkeit eines Referenzstandards für den Abgleich der Ergebnisse der Testsysteme verschiedener Anbieter. Weiterhin verdeutlicht die Variabilität der Messergebnisse die Notwendigkeit einer profunden Assay-Validierung.

\section{P4.6}

\section{A comparison of microcrystalline tyrosine (MCT) and aluminium as adjunvants for allergen immunotherapy in mice}

\section{Leuthardt', A. Duda', E. Contassot', M. Kramer², M. Skinner ${ }^{3}$, T. Kündig', M. Heath ${ }^{3}$, P. Johansen ${ }^{1}$}

${ }^{1}$ Department of Dermatology, University of Zurich and University Hospital Zurich, Zurich, Switzerland

${ }^{2}$ Bencard Allergie GmbH, Munich, Germany

${ }^{3}$ Allergy Therapeutics, United Kingdom

Introduction: Allergen immunotherapy (AIT) is potentially disease modifying. However, low efficacy, long treatment duration, and frequently occurring side effects limit AIT usage and treatment adherence. One strategy to improve AIT is to use new and safer adjuvants. We compared AIT efficacy using microcrystalline tyrosine (MCT) or aluminium (Alum) adjuvants and analysed and compared their mode of action.

Methods: Female BALB/c mice were sensitised by multiple low-dose intraperitoneal injections with cat allergen extract. After f4 weeks, mice received subcutaneous AIT comprising recombinant cat major allergen ( $\mathrm{rFel} \mathrm{d} \mathrm{1)} \mathrm{using} \mathrm{MCT} \mathrm{or} \mathrm{Alum.}$ The AIT was repeated twice with two-week intervals. Cat-dander- and Fel d 1-specific antibodies were tested in serum, and AIT efficacy was tested in a mouse model of allergic anaphylaxis. Potential mechanisms of the mode of action of MCT were studied using mice deficient in Toll-like receptor (TLR) and inflammasome signallingB- and T-cell responses were analysed by ELISAs and flow cytometry.

Results: AIT with rFel d 1 and either MCT or Alum induced comparable IgG1 and IgG2a responses. Antigen-specific IgE response slightly decreased after AIT with MCT, but not after Alum-based AIT. MCT and Alum comparably reduced symptoms of allergic anaphylaxis in sensitised mice. MCT and Alum induced comparable secretion of the Thl cytokines IL-2 and, IFN- $\gamma$, while MCT induced less of the mouse Th-2-like cytokines IL-4 and IL-10. Neither B- nor T-cell responses induced with MCT- or Alum-based vaccines were dependent signalling associated with TLRs or with inflammasome activation.

Conclusions: MCT and Alum are effective adjuvants in immunisation and AIT with MCT triggering less Th2-like immune responses with less IgE. The induced B- and T-cell responses are comparable and independent of TLR signalling and inflammasome activation. Hence, MCT represents a suitable alternative depot adjuvant to Alum in AIT.

\section{P4.7}

Microcrystalline tyrosine (MCT), a promising depo T-forming adjuvant for effective $\mathrm{T}$ cell response invirus-like particle-based vaccines in melanoma murine models

\section{O. Mohsen 1, 2, C. Lipp², M. Heath ${ }^{3}$, M. Skinner ${ }^{3}$, M. Kramer ${ }^{4}$, J. Stein ${ }^{5}$, M. Bachmann ${ }^{1,2}$}

${ }^{1}$ Jenner Institute, Nuffield Department of Medicine, University of Oxford, Oxford, United Kingdom

${ }^{2}$ Immunologie, Inselspital, Universitätslinik RIA, Bern, Switzerland

${ }^{3}$ Allergy Therapeutics, Worthing, United Kingdom

${ }^{4}$ Bencard Allergie GmbH, Munich, Germany

${ }^{5}$ Theodor Kocher Institute, University of Bern, Bern, Switzerland

Introduction: Enhancing the immunogenicity of $\mathrm{T}$ cell vaccines without compromising their tolerability \& safety is an inherent dilemma for vaccine development. It is still unclear whether aluminium hydroxide (Alum) adjuvants can effectively stimulate cytotoxic $\mathrm{T}$ cell (CTL) responses as research suggests that Alum preferentially induces type 2 immunity. Microcrystalline tyrosine (MCT) is a depot adjuvant that has been used in licensed products and subject to further clinical development in allergy immunotherapy. Combining MCT with target antigen (ag) facilitates the slow release of ag while MCT particles are metabolized and cleared from the body. The current project aims to study the efficacy of MCT when combined with viruslike particle (VLP) based vaccines for effective T cell responses in murine melanoma models and compare its efficacy to the widely used Alum adjuvant.

Methods: Vaccines were generated using cucumber mosaic virus derived VLPs coupled to the p33 CTL epitope from LCMV as a model antigen, using bio-orthogonal $\mathrm{Cu}$-free click chemistry. The developed vaccine was formulated with MCT or Alum to test their efficacy at inducing specific $\mathrm{T}$ cell response in vivo in murine models bearing B16F10 melanoma tumors. The kinetics of drainage of VLPs formulated with MCT adjuvants was also studied using stereomicroscopic imaging.

Results: Our results show a superior ability of VLPs formulated with MCT at inducing p33 specific T cell response compared to VLPs formulated with Alum. Frequency of CD8+ IFN- $\gamma+$ cells were significantly increased when combining the VLP-p33 vaccine with MCT compared to Alum. The stereomicroscopic imaging of the drainage of VLPs when formulated with MCT was significantly delayed when compared to free VLPs, demonstrating a depot effect.

Conclusions: MCT is a promising depot adjuvant which enhances CTL responses in murine models. P33 specific T cell response and the production of IFN- $\gamma$ were significantly enhanced when formulating VLPs with MCT. 
P4.8

\section{Entwicklung eines kompetitiven ELISAs zur Bestimmung der IgG-basierten Aktivität in Allergoiden und Aluminium-adsorbierten Allergoid-Präparaten aus Gräser-, Birken-, Beifuß- und Ölbaumpollen}

\section{Schönknecht, K. Bauermeister, G. Reese, M. Stock, V. Scharff, K. Engler, C. Willers, S. Augustin}

Allergopharma GmbH \& Co. KG, Reinbek, Deutschland

Hintergrund: Chemisch-modifizierte Allergene (Allergoide) zeichnen sich durch eine reduzierte IgE-Bindung unter Beibehaltung der therapeutischen Potenz aus. Immunologische Methoden, die zur Charakterisierung von nativen Allergenen verwendet werden, sind zur Charakterisierung von Allergoiden oft nicht anwendbar. Daher fordert die Guideline on Allergen Products: Production and Quality Issues eine weitere Methode zur Bestimmung der IgG-Reaktivität der Allergoide. Dafür wurde ein Inhibitions-ELISA entwickelt, mit dem die relative, spezifische IgG-basierte Aktivität von Allergoiden und Allergoid-Adsorbaten bestimmt werden kann.

Methode: Allergoid-spezifische Seren wurden durch Immunisierung von Ziegen und Kaninchen mit Allergoiden aus Gräser-, Birken-, Beifuß- und Ölbaumpollen gewonnen. Gut charakterisierte Allergoide dienen als Referenz. Die entwickelte Methode ist ein indirekter kompetitiver ELISA, bei dem der Inhibitor (Referenz oder Probe) mit an der Festphase immobilisierten Allergoiden um die Bindungsstellen von Allergoid-spezifischen IgG-Antikörpern konkurriert. Durch das enzymatische Detektionssystem bestehend aus HRP/TMBSubstrat wird die optische Dichte bestimmt, die umgekehrt proportional zur Aktivität des Inhibitors ist. Der Assay wurde hinsichtlich der Parameter Linearität, Richtigkeit, Präzision und Robustheit untersucht.

Ergebnisse: Die Serum- und Referenzverdünnung sowie der Probenverdünnungsfaktor wurden so gewählt, dass mindestens sechs Datenpunkte innerhalb des „pseudolinearen“ Bereichs (20-80\% Inhibition) der Inhibitionskurve liegen. Der Arbeitsbereich wurde für alle untersuchten Allergoide definiert und wies eine sehr gute Linearität $(r>0,9996)$ auf. Die Wiederfindungsraten lagen bei 98-106\% und die Variationskoeffizienten bei $<10 \%$.

Schlussfolgerung: Es wurde ein robuster und präziser Inhibitions-ELISA entwickelt, mit dem die IgG-basierte Aktivität von Allergoiden und Allergoid-haltigen Präparaten bestimmt werden kann.

\section{$\mathbf{P} 4.9$}

Maschinelles Lernen zur Evaluierung von IgE- und IgG4-Antworten als patientenspezifisches $\mathrm{Ma} B$ der Exposition gegenüber der sublingualen Allergie Immuntherapie

\section{T. Stranzl'1 , C. Lundegaard'1 , P. Andersen ${ }^{2}$}

${ }^{1}$ Bioinformatics, ALK A/S, Hørsholm, Dänemark ${ }^{2}$ Immunology, ALK A/S, Hørsholm, Dänemark

Hintergrund: Die Allergie Immuntherapie induziert einen Anstieg allergen-spezifischer Antikörperspiegel. Unser Ziel war es, die Relevanz des allergen-spezifischen IgE (sIgE) und IgG4 (sIgG4)-Anstiegs als patienten-spezifisches Maß der Exposition mit der sublingualen Tabletten-Immuntherapie (SLIT-Tablette) zu evaluieren.

Methoden: Maschinelles Lernen wurde eingesetzt, um zwischen Patienten mit aktiver Behandlung bzw. Placebo zu differenzieren (Algorithmus mit sIgE- und sIgG4-Messungen bei Baseline und 1 Follow-up-Visite, Allergentyp, Behandlungswoche, Patientenalter und Saison-Bezug für saisonale Allergene). Daten aus 8 placebo-kontrollierten Phase III-Studien mit schnell-auflösenden SLIT-Tabletten (2796 Patienten) bei allergischer Rhinitis und/oder Asthma gegen Hausstaubmilben, Gräser und Ragweed mit sIgE- und sIgG4-Daten bei Baseline und allen Visiten von 3-57 Wochen Behandlung ( $N=13900$ Visiten) wurden eingeschlossen.

Ergebnisse: In der aktiv behandelten Gruppe zeigten sich konsistente sIgE- und sIgG4-Anstiege. Mit maschinellem Lernen ließen sich Patienten mit aktiver Therapie versus Placebo während des 1 . Behandlungsjahres differenzieren. Die Anwendung des Algorithmus für verschiedene Zeitpunkte im 1. Behandlungsjahr zeigte konsistente Vorhersagewerte mit einer Genauigkeit von $88 \%$. Patienten unter Aktivtherapie aber mit untypisch niedriger Antikörperantwort (falsch negativ), vergleichbar Placebo, hatten ebenfalls eine geringere Häufigkeit unerwünschter Ereignisse. Die immunologische Antwort zeigte keine konsistente Korrelation mit dem primären klinischen Endpunkt der Studien.

Schlussfolgerung: Auf Patientenebene könnte das Ausmaß der Veränderung des allergen-spezifischen IgE und IgG4 eingesetzt werden, um die aktive Behandlung mit schnell-auflösenden SLIT-Tabletten während des ersten Therapiejahres, trotz der zugrundeliegenden Heterogenität der eingeschlossenen Patienten, der Allergiebehandlung und der Studienprotokolle, vorherzusagen.

\section{P4.10}

\section{Entwicklung eines fluoreszenzbasierten Assays zur Bestimmung des Grades der Allergoidisierung}

\section{Wald, S. Rennert, A. Cossé, C. Willers, S. Augustin}

Allergopharma GmbH \& Co. KG, Reinbek, Deutschland

Hintergrund: Allergoide sind chemisch modifizierte Extrakte aus Allergenquellen und werden als Wirkstoffe in der spezifischen Immuntherapie eingesetzt. Das Ziel der Allergoidisierung ist es, die Allergenität der Extrakte zu reduzieren und die Fähigkeit zur Toleranzinduktion aufrecht zu erhalten. Häufig verwendete Chemikalien sind Glutaraldehyd (GA), Formaldehyd (FA) und FA/GA-Mischungen. Aldehyde bewirken eine Modifikation von Aminogruppen. Zur Bestimmung des Grades der Modifikation von Allergoiden wurde ein Assay etabliert, der auf der quantitativen Bestimmung von intakten Aminogruppen beruht.

Methoden: OPA(o Phthaldialdehyd)-Reagenz wurde $\mathrm{zu}$ Standards (BSA) oder Proben in Mikrotiter-Platten gegeben und das fluoreszierende Endprodukt der Reaktion von OPA mit Aminogruppen quantifiziert. Der „normalized amine value" (NAV) einer Probe ist definiert als das Verhältnis der Fluoreszenzintensität einer Probe zu einem plattenspezifischen Referenzwert („B-Ref“) beruhend auf den Standards. Nach Etablierung des Assays wurden verschiedene Extrakt- und Allergoid-Chargen (Herstellungschargen von Allergopharma) aus Milben, Gräser- und Baumpollen analysiert. 
Ergebnisse: Die Fluoreszenzintensität war bis zu einem Proteingehalt von $10.000 \mathrm{PNU} / \mathrm{mL}$ linear. Der Variationskoeffizient der „Intermediate Precision“ (Messungen an 3 Arbeitstagen) war $<14 \%$. Spiking-Experimente von Extrakt mit Allergoid bewiesen, dass eine quantitative Bestimmung von Aminogruppen in Anwesenheit von Allergoid möglich ist. Die analysierten Allergoide wiesen ca. 4 \% (FA) und 3 \% (FA/GA) der in einem Extrakt vorhandenen Aminogruppen auf und zeigten nur sehr geringe Chargen-Unterschiede.

Schlussfolgerung: Die getesteten Allergoide zeichneten sich durch einen hohen Allergoidisierungsgrad und eine hohe Chargen-Vergleichbarkeit aus. Der Assay ist unkompliziert, schnell und präzise und kann sowohl für Forschungszwecke als auch für die Qualitätskontrolle von Wirkstoffen eingesetzt werden.

\section{P4.11}

\section{Standardisierte Erfassung der}

allergenprovozierten kutanen Soforttyp Reaktion

bei atopischer Dermatitis in der GA2LEN

Pollenkammer: eine Pilotstudie

\section{J. Fluhr, J. Salame, S. Al-Suwaidi, T. Zuberbier}

Department of Dermatology and Allergy, Charité Universitätsmedizin Berlin, Berlin, Deutschland

Hintergrund: Die GA ${ }^{2}$ LEN Pollenkammer wurde entwickelt, um unter standardisierten Bedingungen eine Pollenbelastung unter Modulation von Umweltbedingungen durchzuführen. Eine Validierung bei Atemwegsallergien mit Inhalationsallergenen ist bereits erfolgt. In der vorliegenden Studie sollte eruiert werden, ob der topisch-kutane Kontakt in Kombination mit aerogener Exposition mit relevanten Allergenen bei Patienten mit atopischer Dermatitis (AD) zu einer kutanen Soforttyp Reaktion führt.

Methode: In der vorliegenden Pilotstudie wurden 6 ADPatienten mit klinisch relevanter Typ I Sensibilisierung der Atemwege gegen Hasel, Birke, Erle, Gräser oder Hausstaubmilbe in die prospektive Studie eingeschlossen ( $3 \mathrm{~m} \mathrm{3w}$; $25-70$ J.). Die Exposition erfolgte mit den jeweils relevanten Allergenen in der Pollenkammer für 90 Minuten wobei Gesicht, Hals und Arme unbekleidet waren. Nach einem Intervall von jeweils 30 Minuten erfolgte eine Erfassung der Beschwerden an Augen, Nase, Lunge und Haut mittels Symptomscore.

Ergebnisse: 5 von 6 Patienten entwickelten nach der Exposition zusätzlich zu Atemwegsbeschwerden Erytheme an den Armen mit Juckreiz und Brennen der Haut. Die kutanen Symptome begannen nach 30 Minuten und erreichten nach 60 Minuten ein Plateau. Erytheme und subjektive Symptomatik bildeten sich bei allen 5 betroffenen Patienten innerhalb von 24 Stunden weitestgehend zurück ohne eine ekzematöse Umwandlung. Nach 48 Stunden waren nur noch nicht-juckende diskrete Erytheme zu sehen. Schwerwiegende Nebenwirkungen traten nicht auf.

Diskussion: Bei der AD treten verschiedene Subtypen auf. Bei einer Untergruppe von Patienten stellt die kutane Exposition mit Allergenen einen Triggerfaktor dar. Initial berichten viele $\mathrm{Pa}-$ tienten über Hautrötungen und Juckreiz nach Allergenkontakt, die sich bei längerem Kontakt ekzematös umwandeln. Die Pilotstudie zeigt, dass die GA ${ }^{2}$ LEN Pollenkammer sich als standardisierte Methode zur Untersuchung des Einflußes von exogenen Allergenen auf den Hautzustand bei AD-Patienten eignet. Diese Methode ist prädestiniert für Studien mit Medikamenten, die auf die Blockade der IgE getriggerten Exazerbation der AD abzielen.

\section{Case reports}

\section{P5.1}

\section{Gefahr durch Pflanzen: Allergien und Irritationen}

\section{A. Bauer}

Klinik und Poliklinik für Dermatologie, Universitätsklinikum Carl Gustav Carus, TU Dresden, Dresden, Deutschland

Pflanzeninhaltsstoffe werden häufig in Kosmetika und naturheilkundlichen Präparaten eingesetzt. Als unerwünschte Wirkungen können neben irritativen und allergischen auch phototoxische und photoallergische Kontaktekzeme nach direktem oder aerogenem Hautkontakt oder hämatogene Kontaktekzeme nach Ingestion auftreten. Schätzungen gehen davon aus, dass ca. $5-10 \%$ der in Europa diagnostizierten Kontaktallergien auf Typ-IV Sensibilisierungen gegen Pflanzeninhaltsstoffe zurückzuführen sind. Andere relevante Kontaktallergene, die bei V.a. Pflanzenallergie differentialdiagnostisch in Erwägung gezogen werden müssen sind Pestizide, aber auch Allergene in Hautschutz- und Pflegeprodukten (Gummiinhaltsstoffe, Duftstoffe, Konservierungsstoffe). Beruflich bedingte allergische Kontaktekzeme gegen Pflanzen und Pflanzeninhaltsstoffe treten vorwiegend bei Gärtnern- und Gartenbauern, Floristen, Land- und Forstwirten, Köchen, Kosmetikern und Apothekern auf. Nach direktem Pflanzenkontakt sind irritative Reaktionen häufiger als allergische. Hautirritationen erfolgen physikalischmechanisch und chemisch-toxisch. Neben verschiedenen Pflanzenfamilien wie Amaryllidaceae, Araceae, Cactaceae, Euphorbiaceae, Ranunculaceae und Solanaceae gibt es eine Reihe von Früchten und Gemüsen, die eine irritative Kontaktdermatitis auslösen können. Nicht-immunologische urtikarielle Soforttypreaktionen werden nach Kontakt zu Brennesseln oder Wolfsmilchgewächsen berichtet. Differentialdiagnostische Schwierigkeiten in der Abgrenzung von irritativen und allergischen Reaktionen können sich ergeben, da vielen Pflanzenfamilien sowohl irritative als auch allergene Potenz aufweisen.

\section{P5.2}

\section{Prävalenz allergischer Kontaktekzeme und Epikutantestergebnisse von Patienten, die an einem modifizierten stationären Heilverfahren zur tertiären Prävention von Berufsdermatosen (TIP) teilnehmen}

\section{R. Brans', C. Schröder-Kraft', C. Skudlik', S. John', J. Geier}

${ }^{1}$ Institut für interdisziplinäre Dermatologische Prävention und Rehabilitation (iDerm), Universität Osnabrück, Osnabrück, Deutschland

${ }^{2}$ Informationsverbund Dermatologischer Kliniken (IVDK), Universität Göttingen, Göttingen, Deutschland

Hintergrund: Das allergische Kontaktekzem ist eine der häufigsten beruflich bedingten Hauterkrankungen. Die Prävalenz der Erkrankung und die auslösenden Allergene sind abhängig von der jeweiligen Exposition am Arbeitsplatz.

Methode: Retrospektiv wurden die IVDK-Daten von 3411 Patienten, die von Januar 2007 bis Dezember 2016 im Institut 
für interdisziplinäre dermatologische Prävention und Rehabilitation (iDerm) an den Standorten Osnabrück und Hamburg an einem modifizierten stationären Heilverfahren zur tertiären Prävention von Berufsdermatosen (TIP) teilgenommen haben, ausgewertet. Dabei wurden die Prävalenz allergischer Kontaktekzeme und die Epikutantestergebnisse in den verschiedenen Berufsgruppen verglichen.

Ergebnisse: Eine beruflich bedingte Hauterkrankung wurde bei 2687 Patienten $(78,8 \%)$ diagnostiziert. Überwiegend waren die Hände betroffen. Hautveränderungen im Gesicht wurden am häufigsten bei Malern und Lackierern $(8,1 \%)$ festgestellt. Allergische Kontaktekzeme fanden sich am häufigsten bei Malern und Lackierern (56,8\%), Friseuren (45,8\%), Beschäftigten im Baugewerbe (31,5\%), Garten- und Landschaftsbauern/Floristen (26,7\%) und Metallarbeitern (26,5\%). Die Patienten aus dem Friseurgewerbe waren bei der TIP-Teilnahme signifikant jünger als die Patienten aus anderen Berufsgruppen (Durchschnittsalter: $31,4 \pm 12,6$ Jahre, $p<0,0001$ ) und zuvor am kürzesten in ihrem Beruf tätig (durchschnittlicher Zeitraum: 13,7 $\pm 12,5$ Jahre). Dies spricht dafür, dass in dieser Berufsgruppe schwere beruflich bedingte Hauterkrankungen bereits sehr früh auftreten. Die Epikutantestergebnisse der TIPPatienten wiesen berufsspezifische Unterschiede auf.

Diskussion: Durch die Identifizierung von Berufsgruppen mit einem besonders hohen Risiko für allergische Kontaktekzeme und der hierfür relevanten Allergene können neue Ansätze für gezielte Präventionsmaßnahmen entwickelt werden. Dabei sollten insbesondere Beschäftigte im Friseurgewerbe sowie Maler und Lackierer berücksichtigt werden.

\section{P5.3}

\section{Allergische Rhinitis - Suche nach der Stecknadel im Heuhaufen}

\section{J. Draf, M. Cuevas}

Abteilung für Allergologie/Rhinologie, Klinik und Poliklinik für HNO-Heilkunde, Universitätsklinikum Dresden, Dresden, Deutschland

Einleitung: Die allergische Rhinitis hat in Deutschland eine Prävalenz von ca. $20 \%$. Dabei sind für die saisonale Rhinitis allergica als häufigste Allergene die Frühblüher, Gräser und Beifuß bekannt und werden daher auch initial getestet. Kasuistik: Wir berichten über eine 44-jährige Patientin ohne Vorerkrankungen, welche sich mit ganzjährigen rhinokonjunktivalen Beschwerden vorstellte. Von Ende Mai bis August komme es zu einer Zunahme der Symptome. Eine subcutane spezifische Immuntherapie für Frühblüher und Beifuß war bereits abgeschlossen. In der HNO-ärztlichen Untersuchung zeigten sich endonasal die Schleimhäute diskret geschwollen. Laborchemisch war bei erhöhtem Gesamt IgE (149 IU/ml) das spez. IgE für Lieschgras, Roggen, Beifuß, Birke, Dermatophagoides pteronissinus und farinae erhöht. Im Hautpricktest zeigte sich für Birke, Erle, Hasel, Buche, Beifuß, Hausstaubmilben ein positiver Befund, für Gräser, Roggen, Alternaria alternata, Cladosporium cladosporides und Aspergillus fumigatus ein Negativer. Der konjunktivale Provokationstest für Gräser, Roggen, Beifuß und Spitzwegerich war negativ. Für Milbe und Brennnessel war er positiv, bei Birke kam es zu leichtem Juckreiz der Augen. Wir indizierten daher die spezifische Immuntherapie für Milben und für Brennesel. Ein Nasaler Provokationstest für Frühblüher steht aus.

Schlussfolgerung: Zur Identifizierung der potenziellen Allergieauslöser ist die dezidierte Anamnese unverzichtbar.
Zudem ist der Aktualitätsnachweis für seltene und ganzjährige Allergene über eine Provokationstestung unerlässlich.

\section{P5.4}

Kontaktallergie auf Isobornylacrylat

in kontinuierlich messendem Glucose-Sensor

\section{J. Feustel, L. Ertl, N. Wagner}

Hautklinik, Universitätsklinik Erlangen, Erlangen, Deutschland

Kontinuierlich messende Glucose-Sensoren, die am Oberarm oder am Abdomen mit einer Klebefläche über mehrere Tage fixiert werden, sind inzwischen, insbesondere bei jugendlichen Typ-1-Diabetikern, weit verbreitet. Sie bedeuten einen deutlichen Zuwachs an Lebensqualität für die Patienten.

Zunehmend wird über Kontaktallergien auf das Fixierungspflaster berichtet. Ein detektiertes, auslösendes Kontaktallergen ist Isobornylacrylat. Es gibt Hinweise, dass Isobornylacrylat aus dem Sensorsystem und nicht aus dem Fixierungspflaster freigesetzt wird. Wir berichten über einen 47-jährigen Patienten, der bereits wenige Tage nach Beginn der Applikation des GlucoseSensors eine Kontaktallergie entwickelte. In der folgenden Epikutantestung mit 0,1 \% Isobornylacrylat in Vaseline, lies sich die Verdachtsdiagnose bestätigen, ohne dass eine Kreuzallergie auf andere Acrylatverbindungen vorlag.

Trotz des Versuches mit Hilfe diverser dünner Wundauflagen, die als „Barriereschicht“ die Auslösung des Kontaktekzems unterbinden sollten, um die Verwendung des Sensors weiterhin zu ermöglichen, bestand das Kontaktekzem fort. Die Auswahl von Materialien, die den Kontakt zur Klebefläche des Sensors reduzieren, ist durch die Nadellänge des Sensors limitiert, da die Zuverlässigkeit der Blutzuckermessung eingeschränkt werden kann.

Der geschilderte Patient musste aufgrund des Fortbestehens des Kontaktekzems auf den kontinuierlich messenden GlucoseSensor verzichten.

\section{P5.5}

Berufsbedingte Schweineproteinallergie vom Typ I; allergische Rhinitis, Kontakturtikaria und Asthma als Berufserkrankungen in einer Grossschlachterei

\section{S. Jungewelter}

Finnish Institute of Occupational Health, Helsinki, Finnland

Wir berichten über vier Arbeiter aus einer Grossschlachterei mit beruflicher Typ-I-Sofort-Allergie gegenüber SchweineproteinAllergenen. Diese Allergien wurden durch positive Hauttests (Skin Prick Testung) auf diverse rohe Schweinsteile wie Fleisch und/oder Innereien, und erhöhte spezifische Immunoglobuline E-Antikörper-Titer im Serum (ImmunoCAP) gegen Schweinefleisch, Schweineurin und/oder Schweineepithel diagnostiziert.

Die verschiedenen Beschwerden der vier Patienten, d.h. recurrenter wässriger Schnupfen, Urtikaria an den Vorderarmen, Husten, Heiserkeit oder Dyspnoe, hatten 1 bis 14 Jahre gedauert, bevor die Patienten in unserer Klinik vorgestellt wurden. Im Schlachtereibetrieb waren diese Arbeiter seit 7 bis 17 Jahren tätig gewesen. Symptome nach oraler Aufnahme vom Schweinefleisch hatte keiner der Patienten angegeben. 
Bei einer Arbeiterin wurde Berufsasthma durch erhöhte bronchiale Hyperreagibilität in einem spezifischen inhalativen Provokationstest mit rohen Schweineinnereien und durch arbeitsplatzbezogene serielle Peak flow(PEF)-Messungen mit niedrigeren PEF-Werten während der Arbeit nachgewiesen. Bei zwei Arbeitern wurde allergische Rhinitis als Berufskrankheit durch positive nasale Provokationstests (NPT) mit einem Extract aus rohem Schweinefleisch diagnostiziert. Einer von diesen Arbeitern hatte auch berufsbedingte Proteinkontakturtikaria, nachgewiesen durch die typische Symptomatik und durch die Sensibilisierung gegenüber Schweinefleisch. Bei der vierten Arbeiterin konnte eine Typ-I-Schweinefleischallergie diagnostiziert werden, jedoch keine typische Berufserkrankung der Haut oder der Atemwege.

Wir möchten auf frühe Zeichen einer SchweinefleischAllergie bei beruflicher Exposition aufmerksam machen, auch um ein besonderes Augenmerk auf die Präventionsarbeit in Schweinefleisch verarbeitenden Betrieben zu richten.

\section{P5.6}

Schwere systemische Anaphylaxie nach Maracuja-Eis bei einer Patientin mit Latex-Allergie

\section{A. J. Kilbertus, I. Angelova-Fischer, W. Hötzenecker}

Dermatologie, Kepler Uniklinikum Linz, Linz, Österreich

Eine erhebliche Anzahl an Patienten mit Latex-Allergie zeigen IgE-vermittelte Reaktionen auf assoziierte Nahrungsmittel im Rahmen eines Latex-Frucht-Syndroms. Eine 43-jährige Patientin wurde an unsere Klinik zur Notfallversorgung und Observanz aufgrund einer anaphylaktischen Reaktion dritten Grades zugewiesen. Die Patientin wollte sich aufgrund der vorherrschenden Hitzeperiode mit dem Verzehr eines MaracujaEis abkühlen. Nach dem Genuss kam es innerhalb weniger Minuten zu plötzlich auftretendem generalisierten Pruritus, Urticaria, Angioödemen der Lippen, Globusgefühl, Luftnot und Übelkeit sowie in weiterer Folge zu Magenkrämpfen, Erbrechen und Diarrhoe. Nach Notfallversorgung mit systemischen Glukokortikoiden, Antihistaminika sowie inhalativem Adrenalin konnte ein vollständiges Abklingen der Symptome erreicht werden und eine Entlassung nach 12 Stunden stationärer Überwachung erfolgen. Anamnestisch kam es auch in der Vergangenheit bereits zu anaphylaktischen Reaktionen nach dem Verzehr einer Avocado, sowie nach einer Feige und einer Papaya. In der ehemaligen Tätigkeit als Pflegehelferin in einem Krankenhaus beschrieb sie Rötungen und Schwellungen an den Händen während bzw. nach der Verwendung von Gummi-Handschuhen. In der in-vitro-Diagnostik zeigte sich bei einem Gesamt-IgE-Wert von $77 \mathrm{U} / \mathrm{ml}$ und normwertiger Tryptase in der spezifischen IgE-Bestimmung gegen Gesamtextrakt Latex Hevea brasiliensis ein Wert von 2,7 kU/1 (Kl.2) sowie gegen das rekombinante Major-Allergen rHev b6.02 Hevein ein Wert von 3,7 kU/1 (Kl.3). Das Latex-Profilin rHev b8 war negativ, ebenso das PR10 Protein rBet vl und das nicht-spezifische Lipidtransferprotein rPru p3. Basierend auf diesen Befunden wurde die Diagnose eines Latex-Frucht-Syndroms gestellt. Während zahlreiche Früchte als häufige Auslöser für Latex-assoziierte NahrungsmittelHypersensitivitätsreaktionen beschrieben wurde, ist Maracuja ein seltener Auslöser für schwere systemische Anaphylaxien im Rahmen eines Latex-Frucht-Syndroms.

\section{P5.7}

Kontaktekzem bei Typ IV - Sensibilisierung auf Isobornylarcylat

\section{Lotz, A. Bauer}

Klinik und Poliklinik für Dermatologie, Universitätsklinikum Karl Gustav Carus, Dresden, Deutschland

Wir berichten den Fall eines 11-jährigen Kindes mit einem Typ I-Diabetes.

Ab dem zweiten Monat nach Erstdiagnose wurde das Sensorsystem Freestyle Libre ${ }^{\bullet}$ zur kontinuierlichen Blutzuckerüberwachung angewendet. Nach 4-wöchiger Anwendung kam es zu einer ersten Ekzembildung im Bereich der Kontaktstelle des Haftmaterials mit der Haut des Oberarmes. Die Hautreaktion war an verschiedenen Lokalisationen beider Arme reproduzierbar. Auch ein Wechsel auf ein Messsystem eines anderen Herstellers erbrachte keine Befundverbesserung. Es wurde folgend der Versuch unternommen, mittels zusätzlicher Anwendung verschiedener Pflaster eine Diffusionssperre zu erzeugen, um den Sensor weiter verwenden zu können. Lediglich mit dem Produkt Stomahesive ${ }^{\otimes}$ war dies erfolgreich.

Wir führten eine umfangreiche Epicutantestung durch. Es zeigte sich eine deutliche Typ IV-Sensibilisierung gegen Isobornylacrylat (Testkonzentrationen $0,1 \%$ und 0,01\%). Bei diesem Stoff handelt es sich um einen Reaktivverdünner, der zur Verbesserung der Viskosität dem Klebstoff zugesetzt wird, mit dem die Einzelteile des Produktes verbunden werden. Dieser verbindet sich folgend im Rahmen einer Copolymerisation mit dem eigentlichen Klebstoff und härtet mit diesem zusammen aus. Dennoch verbleiben monomere Reste dieses Acrylates, die durch das gesamte Produkt incl. dem Haftpad diffundieren und hier an der Kontaktstelle zur Epidermis Sensibilisierungen generieren können.

Seit der Erstbeschreibung einer Sensibilisierung durch eine Insulinpumpe im Jahr 1995 (Busschots et al.) und durch ein kontinuierliches Blutzuckersensorsystem im Jahre 2017 (Hermann et al.) kommt es derzeit mit der häufigeren Anwendung solcher Systeme zu einer zunehmenden Zahl von Sensibilisierungen auf Isobornylacrylat.

Kreuzreaktivitäten $\mathrm{zu}$ anderen Acrylaten wurden bisher nicht beschrieben.

\section{P5.8}

Urtikariavaskulitis, Churg-Strauss-Syndrom, Schnitzler-Syndrom oder doch chronisch spontane Urtikaria?

\section{G. Meinel, S. Abraham, A. Bauer}

Klinik und Poliklinik für Dermatologie, Universitätsklinikum Karl Gustav Carus, Dresden, Deutschland

Urtikarielle Erkrankungen können eine differentialdiagnostische Herausforderung darstellen.

Eine 59-jährige Patientin stellte sich mit seit 6 Monaten rezidivierend am ganzen Körper auftretenden Urticae, die mit deutlichen Hyperpigmentierungen abheilten, vor. Sie gab eine Verschlechterung des bekannten nicht-allergischen Asthma bronchiale an, die sich auch in einer auswärtigen Bodyplethysmographie zeigte. Eine 1 Jahr zuvor durchgeführte Lungenbi- 
opsie war unauffällig gewesen. Nebendiagnostisch bestanden eine chronische Pansinusitis, Schmerzen im Bereich der Extremitätengelenke sowie der V.a. eine NSAR-Intoleranz. Fieberschübe wurden nicht berichtet. Im Blutbild war eine Erhöhung der Eosinophilen (2,36 GPt/l) aufgefallen. Die Fokussuche ergab keine Ursache für die Eosinophilie.

Eine Hautbiopsie zeigte eine mit einer Urtikariavaskulitis $\mathrm{zu}$ vereinbarende eosinophilenreiche Vaskulitis. Direkte und indirekte Immunfluoreszenz einschließlich ANCA sowie C3, C4 und die Serumeiweißelektrophorese gestalteten sich jedoch unauffällig. In der Blutbildkontrolle zeigten sich mit 0,61 GPt/1 nur noch leicht erhöhte Eosinophile. Die Immunfixation im Serum ergab eine schwache monoklonale Gammopathie IgG Typ Lambda mit normwertigem k/l-Leichtketten-Index. Eine erneute Lungenfunktionsprüfung gestaltete sich unauffällig.

Unter Fexofenadin $180 \mathrm{mg}$ 1-0-2 traten im Verlauf keine Quaddeln mehr auf, weiterhin kam es zu einem deutlichen Abblassen der Hyperpigmentierungen.

In Zusammenschau der Befunde ist bei der Patientin trotz atypischer Klinik und in der konventionellen Histologie diagnostizierter mit einer Urtikariavaskulitis $\mathrm{zu}$ vereinbarenden eosinophilen Vaskulitis bei gutem Ansprechen auf eine Antihistaminikadauertherapie von einer chronisch spontanen Urtikaria auszugehen. Dies unterstreicht die Komplexität urtikarieller Hauterkrankungen und weist die Grenzen der konventionellen Histologie in der Urtikariadiagnostik auf.

\section{P5.9}

\section{Auf der Suche nach der Doppelsensibilisierung - Ein Fallbericht}

\section{S. Menzel, M. Cuevas, T. Zahnert}

Klinik für Hals-, Nasen- und Ohrenheilkunde, Universitätsklinik Dresden, Dresden, Deutschland

Einleitung: Ein Viertel aller anaphylaktischer Reaktionen werden durch Insektengiftallergien verursacht. Die Prävalenz hierfür liegt in Europa bei 3,5\%. Eine kausale spezifische subcutane Immuntherapie (SCIT) hat eine Erfolgschance von 90-95\% bei Wespengift und 75-85\% bei Bienengift.

Fallbeispiel: In unserer Klinik stellte sich eine 64-jährige Patientin mit einer bekannten Wespengiftallergie aufgrund eines stattgehabten Hummelstichs in die Hand mit nachfolgender Übelkeit und Dyspnoe vor (Ring-2-Reaktion). Nach der Anwendung des Notfallsets sei keine weitere Behandlung erfolgt. Die bekannte Wespengiftallergie wurde mit einer SCIT über 5 Jahre therapiert. Unter der Therapie habe sie auf einen Wespenstich anaphylaktisch reagiert (Ring-2-Reaktion). Es bestand ein Therapieversagen bzgl. der Wespengiftallergie sowie der V.a. eine Bienengiftallergie. Im Haut-Prick-Test stellte sich ein positiver Befund für Wespengift und ein negativer Befund für Bienengift dar, bei einem positiven Befund im Intrakutan-Test für Bienengift. Laborchemisch zeigte sich bei einem normwertigem gesamt-IgE und einer normwertigen Mastzelltryptase eine Erhöhung des spezifischen IgE für Wespengift. Die spezifische Rekombinante für Wespengift Ves v5 war positiv bei einem unsicheren Befund für Bienengift (Api m5 0,19kU/1). Es erfolgte ein basophiler Aktivierungstest, wobei eine Sensibilisierung durch Wespengift nachweisbar war jedoch nicht für Bienengift. Für die nicht nachweisbare Bienengiftallergie besteht keine therapeutische Konsequenz. Für die Wespengiftallergie besteht die Indikation zur Ultra-Rush-SCIT mit einer Dosissteigerung im Intervall auf $200 \mu \mathrm{g}$ und anschließender Stichprovokation.
Schlussfolgerung: Die Anamnese ist eine wichtige Grundlage zur Diagnostik einer Insektengiftallergie, aufgrund der Ähnlichkeit der Insekten kann sie zu falschen Angaben führen. Bei Therapieversagen sollte eine Dosissteigerung erfolgen. Eine Stichprovokation kann zur Therapiekontrolle eingesetzt werden.

\section{P5.10}

\section{Omalizumab bei 3 Patienten mit schwerer vernaler Keratokonjunctivitis (VKC)}

\section{S. Rossberg', U. Pleyer' ${ }^{2}$, S. Lau ${ }^{1}$}

${ }^{1}$ Klinik für Pädiatrie mit Schwerpunkt Pneumologie, Immunologie und Intensivmedizin, Charité Universitätsmedizin Berlin, Berlin, Deutschland ${ }^{2}$ Klinik für Augenheilkunde, Charité Universitätsmedizin Berlin, Berlin, Deutschland

Hintergrund: Die VKC ist eine pädiatrische Augenerkrankungen. Trotz Standardtherapie können therapierefraktäre Verläufe eintreten. Die entzündliche Reaktion ist oft IgE vermittelt, sodass Omalizumab (Anti-IgE) eine Behandlungsoption ist.

Methoden: Retrospektive Analyse von 3 Fällen mit VKC, die nicht auf Standardtherapie angesprochen haben.

Ergebnisse: 3 männliche Patienten mit früher allergischer Rhinokonjunktivitis (ARK) (1-3 Jahre) und Polysensibilisierung. Komorbiditäten: allergisches Asthma, atopische Dermatitis.

Pat.A: 4. LJ saisonale VKC. Behandlung: Systemische/topische Antihistaminika, topische Kortikosteroide, Cyclosporin A. 10. LJ VKC Stadium IV (konjunktivale Papillen, Trantasflecken), gesamt IgE 766 kU/l, Anti-IgE 6 Monate alle 2 Wochen 300 mg sc. Remission zu VKC Stadium I.

Pat.B: 1. LJ saisonale VKC stage III (konjunktivale Papillen, limbale Irritation). Behandlung: Systemische/topische Antihistaminika, topische Kortikosteroide, Cyclosporin A. 7. LJ VKC Stadium IV, gesamt IgE $1753 \mathrm{kU} / \mathrm{l}$, Anti-IgE 6 Monate alle 2 Wochen $150 \mathrm{mg}$ sc. Remission zu VKC Stadium I.

Pat.C: 5. LJ perenniale VKC Stadium V (konjunktivale Papillen, schwere Hornhauterosionen). Behandlung: Systemische/ topische Antihistaminika, topische Kortikosteroide, Cyclosporin A, Amnionmembran Transplantation. Gabe von syst. Cyclosporin A bei VKC und schwerer atopischer Dermatitis. Kein Ansprechen VKC. 7. LJ gesamt IgE 1295 kU/l, Anti-IgE 6 Monate alle 2 Wochen $300 \mathrm{mg}$ sc. Nach 1. Gabe Ansprechen: Beendigung systemisches Cyclosporin A. Bei Verschlechterung an Haut und Augen, Wiederbeginn Ciclosporin A systemisch. Verbesserung zu VKC Stadium II.

Schlussfolgerung: 3 VKC Patienten zeigten früh eine ARK, Polysensibilisierung, hohes Gesamt IgE und atopische Komorbiditäten. Berichtenswert ist das schnelle Ansprechen auf AntiIgE bei bislang wenig publizierten Fällen. Es bedarf kontrollierter Studien mit Omalizumab, um den Stellenwert als second line Behandlungsoption der VKC weiter zu evaluieren. 
P5.11

\section{Wen der Nymphensittich stört}

\section{S. Wanske ${ }^{1}$, K. Stamos ${ }^{1}$, G. Hahn ${ }^{2}$, C. Vogelberg ${ }^{1}$}

${ }^{1}$ Fachbereich Kinderpneumologie/Allergologie, Klinik und Poliklinik für Kinder- und Jugendmedizin, Universitätsklinikum Carl Gustav Carus, Dresden, Deutschland

${ }^{2}$ Bereich Kinderradiologie, Institut und Poliklinik für Radiologische Diagnostik, Universitätsklinikum Carl Gustav Carus, Dresden, Deutschland

Wir berichten über ein 11 Jahre altes Mädchen, welches mit starkem Husten, erschwerter Atmung und Fieber in einem peripheren Krankenhaus aufgenommen wurde. Wegen fehlender Besserung unter antibiotischer Therapie und einer zunehmenden respiratorischen Insuffizienz erfolgte die Verlegung in unsere Klinik zur weiteren Diagnostik.

Bereits seit ca. 5 Monaten bestanden eine zunehmend verminderte Belastbarkeit, ein Gewichtsverlust von $4 \mathrm{~kg}$ und eine prolongierte pulmonale Symptomatik mit mehreren Pneumonien. Seit einem Monat war kein Schulbesuch mehr möglich aufgrund von anhaltender Atemnot.

Die Lungenfunktion bei Aufnahme zeigte eine schwerste restriktive Ventilationsstörung (VC $13 \%$ ). Im Thorax-CT konnten diffuse air space noduli in allen Lungenlappen und streifige Fibrosierungen in beiden Unterlappen nachgewiesen werden. In der bronchoalveolären Lavage war eine Lymphozytose zu sehen. Da die Familie seit 4 Jahren einen Nymphensittich als Haustier hatte, der im Schlafzimmer des Mädchens untergebracht war, erfolgte die Bestimmung von spezifischen IgG-Antikörpern. Diese ergab eine ausgeprägte IgG-Sensibilisierung gegen Wellensittichkot und -federn.

In Zusammenschau der Befunde gingen wir von einer subakut bis chronischen exogen allergischen Alveolitis (EAA) aus, die durch den Nymphensittich ausgelöst wurde. Die Therapie erfolgte mit hochdosiertem Prednisolon, woraufhin es bereits nach wenigen Tagen zu einer deutlichen Verbesserung der Lungenfunktion und der subjektiven Belastbarkeit kam. Der Sauerstoffbedarf war ebenso rasch rückläufig.

Die EAA ist eine allergische Überreaktion der Lunge auf eingeatmete Partikel, gegen die der Betroffene sensibilisiert ist. Das Krankheitsbild ist weiterhin nur wenig bekannt, so dass die entsprechende Verdachtsdiagnose häufig erst mit einer großen Latenz gestellt wird. Bei einem langsamen Leistungsabfall mit Gewichtsabnahme und zunehmender Luftnot sollte insbesondere bei Haltung von Vögeln an die EAA gedacht werden.

\section{Asthma, upper respiratory tract and hymenoptera venom allergy}

\section{P6.1}

Immuntherapie eines Kindes mit Bienengiftallergie mit prädominanter Api m 10 Sensibilisierung und anhaltend erhöhtem Serumtryptasesespiegel

\section{Buss, L. Junk, C. Kemen, J. Lemke}

Kinderkrankenhaus Wilhelmstift, Hamburg, Deutschland
Anamnese: Wir berichten über einen 8-jährigen Imkersohn mit einer Bienengiftallergie. Bereits im Alter von 4 Jahren reagierte der Junge nach einem Bienenstich in den Fuß mit einer anaphylaktischen Reaktion Grad 2. Bei zwei Folgestichen im Verlauf blieb eine anaphylaktische Reaktion aus, nach einem dritten in den Fuß entwickelte der Junge Urtikaria, Angioödem und Unruhe. Wespenstiche wurden stets reaktionslos vertragen.

Diagnostik: Die Allergiediagnostik ergab eine Sensibilisierung auf Bienengift und die Allergenkomponenten Api ml, Api m3 und Api m10, wobei die Sensibilisierung auf Api m10 prädominierte. Die basale Serumtryptase wurde zweimal bestimmt und ergab jeweils erhöhte Spiegel (18,5 $\mu \mathrm{g} / \mathrm{l}, 19 \mu \mathrm{g} / \mathrm{l})$ ohne klinische Hinweise auf eine Mastozytose. Es wurde eine Immuntherapie eingeleitet mit einem Lyophilisat der Firma Bencard. Für die Fortsetzungsbehandlung wurde auf ein Depotpräparat (ALK Depot) gewechselt. Die Immuntherapie wird seit 18 Monaten gut toleriert. Zu weiteren Stichereignissen ist es nicht gekommen.

\section{Diskussion/offene Fragen:}

1. Für Patienten mit Insektengiftallergie und anhaltend erhöhtem basalen Tryptasspiegel ohne Hinweise auf Mastozytose ist die Immuntherapie besonders wichtig und soll bei Erwachsenen nicht nur für einige Jahre sondern dauerhaft, ggf. lebenslang, erfolgen. Wie lange soll unser Patient therapiert werden?

2. Laboruntersuchungen konnten zeigen, dass der Gehalt an Api m10 in Lyophilisaten verschiedener Hersteller unterschiedlich hoch ist. Enthält der verwendete Depot-Extrakt Api m10 in ausreichender Menge? Sollte im Verlauf der Therapie ein Nachweis der Wirksamkeit durch Bestimmung der IgG4-Antikörper gegen Api m10 erfolgen?

3. Soll bei unserem Patienten mit prädominanter Api m10 Sensibilisierung die klinische Wirksamkeit ausnahmsweise mittels Stichprovokation nachgewiesen werden?

\section{P6.2}

\section{Omalizumabtherapie bei Patienten mit einer nasalen Polyposis - Follow-Up von 12 Monaten}

\section{U. Förster-Ruhrmann', D. Stergioudi' ${ }^{1}$, K. Bergmann², H. Olze ${ }^{1}$}

${ }^{1}$ HNO-Klinik, Charité Universitätsmedizin, Berlin, Deutschland ${ }^{2}$ Allergiezentrum, Charité Universitätsmedizin, Berlin, Deutschland

Hintergrund: Die Therapie der chronischen Rhinosinusitis mit einer nasalen Polyposis (CRSwNP) wird bei komorbidem Asthma und einer ASS-Intoleranz häufig durch rezidivierende nasale Polypen erschwert. Neue Therapieansätze sind Biologika. Wenig ist bislang bekannt über Langzeittherapien von dem Anti IgE-AK Omalizumab bei der nasalen Polyposis, was im Folgenden über einen Zeitraum von 12 Monate untersucht wurde.

Methode: In die prospektive Langzeitstudie einer Omalizumabtherapie wurden 12 Patienten mit einer nasalen Polyposis (NP) eingeschlossen (6w, $6 \mathrm{~m}$, MW 48 Jahre). Ausgewertet wurden die Häufigkeiten der Komorbiditäten, der nasale Polyposis(NP)-Score, das Ansprechen und die Verträglichkeit der Therapie.

Ergebnisse: Die Therapie erfolgte mit $\emptyset 300 \mathrm{mg}$ Omalizumab. In $100 \%$ der Fälle bestand ein Asthma, perenniale Allergien in $50 \%$ und eine chronische Urtikaria in $30 \%$ der Fälle. Vorab waren ø 4 NNH-OPs erfolgt. Der NP-Score war im Verlauf der Follow-Up Untersuchung signifikant reduziert $(p=0,02)$, 
die chronische Urtikaria war komplett regredient, Asthmaexazerbationen bestanden nicht. Eine NNH-OP erfolgte bei einem der Fälle während der Omalizumabtherapie. Hinsichtlich der Verträglichkeit der Therapie zeigten sich bei einem Patienten initial vermehrte Infekte nach den Injektionen, ein Abbruch der Therapie war nicht erforderlich.

Schlussfolgerungen: Die Therapie mit Omalizumab ist effektiv in der Langzeittherapie von Patienten mit einer NP, was in größeren Studien untersucht werden sollte.

\section{P6.3}

\section{Effektivität der Akupunktur \\ bei Nicht-Allergischer-Rhinitis (NAR)}

\section{D.E. Kretschmann' ${ }^{1}$, A. Hähner², B. Hauswald ${ }^{3}$}

${ }^{1}$ HNO-Klinik, Universitätsklinikum Carl Gustav Carus Dresden, TU Dresden, Dresden, Deutschland

${ }^{2}$ Abteilung für Riechen und Schmecken, HNO-Klinik, Universitätsklinikum Carl Gustav Carus Dresden, TU Dresden, Dresden, Deutschland

${ }^{3}$ Allergologieabteilung, HNO Klinik, Universitätsklinikum Carl Gustav Carus Dresden, TU Dresden, Dresden, Deutschland

Hintergrund: Diagnostik und Medikation der NAR sind noch uneinheitlich und aufgrund des heterogenen Patientenguts häufig frustran. Heutzutage sind viele Patienten an komplentärmedizinischen Therapien interessiert, speziell die Akupunktur erlebt eine große Nachfrage. Mit der einfachverblindeten, placebokontrollierten, randomisierten Studie soll erforscht werden, ob Akupunktur die Symptome der NAR verbessert und somit eine weitere Therapiemöglichkeit bei Refrakterität auf medikamentöse Behandlung darstellt.

Methoden: 40 Patienten mit NAR wurden in die Studie eingeschlossen und randomisiert zwei Gruppen (Verum- und Placebo-Akupunktur) zugeordnet. Alle Patienten erhielten 12 Akupunktur-Behandlungen. In der Verum-Gruppe wurden die Punkte LG 20, Di 20, Ex-KH 3, Op 55,Op 29b, KG 17, Lu 7, Di 4, Dü 3, Ma 36, MP 6, Ma 44, Gb 39, Le 3 akupunktiert. Für die Auswahl der Punkte der Placebo-Gruppe diente die ACUSARStudie [Brinkhaus B et al. Acupuncture in Seasonal Allergic Rhinitis(ACUSAR)-design and protocol of a randomised controlled multi-centre Trial. Forsch Komplement med 2010;17:9510] als Vorlage: Deltoideus, Oberarm, Oberschenkel 1-3. Die Punkte Rücken 1-2 wurden aufgrund der Nähe zum Blasenmeridian abgewandelt und lateral in Verlängerung der Nabelmitte akupunktiert. Zusätzlich erfolgte die Placeboakupunktur nur oberflächlich, um die Reizwirkungen minimal zu halten. Bei der ersten, sechsten und zwölften Visite erfolgte eine Riechtestung mit dem Sniffin' Sticks-Test, bestehend aus Schwellen-, Diskriminations- und Identifikationstest. Zur Objektivierung der Symptome erhielten die Patienten weiterhin bei vier Visiten folgende Fragebögen: RQLQ; Nasal-Symptom-Score; und BDI (nur 1. Visite); es wurde ebenfalls im Verlauf mehrfach ein VASScore zur Beschwerdesymptomatik erhoben.

Ergebnisse: Noch ausstehend (voraussichtlich August 2018 verfügbar).

Zusammenfassung: In der vorliegenden Studie wird erforscht, ob Akupunktur eine mögliche Behandlungsform der NAR darstellt.

\section{P6.4}

Dupilumab in patients with corticosteroiddependent severe asthma: efficacy and safety results from the randomized, double-blind, placebo-controlled phase 3 Liberty Asthma Venture study

\section{K. F. Rabe ${ }^{1}$, P. Nair ${ }^{2}$, G. Brusselle ${ }^{3}$, J. Maspero, M. Castro ${ }^{5}$, H. Zhu ${ }^{6}$, H. Staudinger ${ }^{6}$, G. Pirozzi ${ }^{6}$, C. Antoni ${ }^{6}$, N. Amin ${ }^{7}$, M. Ruddy ${ }^{7}$, B. Akinlade ${ }^{7}$, A. Khan ${ }^{6}$, J. Chao ${ }^{7}$, N. Graham ${ }^{7}$, Ariel Teper ${ }^{6}$}

${ }^{1}$ LungenClinic Grosshansdorf, Christian Albrechts University, Großhansdorf, Germany

${ }^{2}$ St. Joseph's Healthcare, McMaster University, Hamilton, Canada

${ }^{3}$ Ghent University Hospital, Ghent, Belgium

${ }^{4}$ Fundación CIDEA, Buenos Aires, Argentina

${ }^{5}$ Washington University School of Medicine, St. Louis, USA

${ }^{6}$ Sanofi, Chilly Mazarin, France

${ }^{7}$ Regeneron Pharmaceuticals, Inc., Tarrytown, NY, USA

Rationale: Dupilumab (DPL), a fully human VelocImmunederived anti-IL-4R $\alpha \mathrm{mAb}$ that inhibits signaling of IL-4/13, key drivers of Type 2 inflammation, significantly reduced severe exacerbations and improved lung function in patients (pts) with uncontrolled persistent asthma (NCT01854047). DPL is approved for treatment of adults with inadequately controlled moderate-to-severe atopic dermatitis. Long-term oral corticosteroid (OCS) use can cause safety risks. This phase 3 study (NCT02528214) evaluates DPL efficacy for OCS reduction in pts with CS-dependent severe asthma.

Method: Pts $\geq 12$ yrs received add-on DPL $300 \mathrm{mg}$ or placebo (PBO) every 2 wks for $24 \mathrm{wks}$. After 8-10 wks optimization, OCS dose was down-titrated from Wk4 to Wk20 and maintained for 4 wks. Primary endpoint: \% reduction in OCS dose at Wk24. Secondary endpoints: absolute reduction in OCS dose; proportion of pts achieving: $\geq 50 \%$ reduction in OCS dose, reduction in OCS dose to $<5 \mathrm{mg} /$ day, and maximum OCS dose reduction at Wk24; and proportion of pts no longer requiring OCS. Other endpoints: annualized severe exacerbation events over the treatment period and change from baseline (BL) in pre-bronchodilator FEV1 at Wks12/24.

Results: In DPL vs PBO pts, OCS dose at Wk24 was reduced by $70.1 \%$ vs $41.9 \%$ from $B L(P<0.0001) ; 80 \%$ vs $50 \%$ achieved $\geq 50 \%$ reduction in OCS, $69 \%$ vs $33 \%$ achieved reduction to $<5 \mathrm{mg} /$ day, $48 \%$ vs $26 \%$ achieved their maximum OCS dose reduction; and $48 \% \mathrm{vs} 25 \%$ no longer required OCS. DPL reduced exacerbations by $59.3 \%(P<0.0001)$ and improved FEV1 by $0.22 \mathrm{~L}$ vs $\mathrm{PBO}$ at Wk24 $(P<0.001)$. Efficacy was observed regardless of baseline minimum blood EOS. Adverse events (AEs) with higher rates in DPL vs PBO were EOS count increase (6.8\% vs. $0 \%)$, eosinophilia (6.8\% vs. $0.9 \%)$, bronchitis ( $6.8 \%$ vs. $5.6 \%)$, and sinusitis (6.8\% vs. $3.7 \%)$. Conjunctivitis AEs were similar across groups ( $1 \%$ vs. $0.9 \%)$

Conclusion: DPL reduced OCS use while reducing exacerbations and improving FEV1 in pts with CS-dependent severe asthma and was generally well tolerated. 
P6.5

Kosten-Nutzwert-Analyse der spezifischen Immuntherapie mit Insektengift in Deutschland

\section{F. Rueff', M. Aagren', I. Thießen ${ }^{3}$, M. Bauer ${ }^{3}$}

${ }^{1}$ Ludwig-Maximilians-Universität, München, Deutschland ${ }^{2}$ ALK-Abelló A/S, Horsholm, Dänemark ${ }^{3}$ ALK-Abelló Arzneimittel GmbH, Hamburg, Deutschland

Hintergrund: Die spezifische Immuntherapie mit Hymenopterengift (HG-SIT) ist hochwirksam, um bei HG-Patienten eine Toleranz ggü. Hymenopterenstichen zu erzeugen. Damit wird zum einen die Lebensqualität (LQ) verbessert und zum anderen können potentiell lebensbedrohliche Stichreaktionen verhindert werden. Hierzu wird eine Kosten-Nutzwert-Analyse durchgeführt. Die Therapiekosten werden in Relation zu der Verbesserung von Parametern (LQ und Verhinderung tödlicher Reaktionen) und den gewonnenen qualitätsadjustierten Lebensjahren (QALYs) gesetzt.

Methoden: Es wurde ein Markov-Modell mit 3 Zuständen zugrunde gelegt: Leben mit Angst vor Stichen (nicht-HG-SIT), angstfrei leben infolge eines therapeutischen Schutzes (HGSIT) und versterben infolge einer Stichanaphylaxie. Die Kostenschätzung basierte auf dem AVP von ALK-depot SQ ${ }^{\circ}$ Insektengiften. Es wurde eine Häufigkeit von 0,095 Stichen/Jahr und Person zugrunde gelegt. Das Risiko an einem Stich zu sterben betrug ohne HG-SIT 0,008\%. Getroffene Annahmen: Alter zur HG-SIT-Einleitung 25 Jahre, normale Lebenserwartung 80,7 Jahre und lebenslange Andauer des Behandlungserfolges. Der Nutzenvorteil durch die reduzierte Angst vor zukünftigen allergischen Reaktionen wurde unter Bezug auf eine frühere NICE-Bewertung mit 0,01 QALYs/Jahr quantifiziert. Die Therapiekosten und QALYs wurden jährlich mit $3 \%$ diskontiert.

Ergebnisse: Die Therapiekosten einer 3-jährigen HG-SIT in Deutschland betragen $4.398 €$. Durch die HG-SIT wurden über die gesamte Lebenszeit 0,27 QALYs hinzugewonnen. Den größten Anteil hierfür hatte die erhöhte LQ aufgrund reduzierter Angst. Die Kosten pro QALY beliefen sich auf $16.415 €$. Die Sensitivitätsanalyse bestätigte ein robustes Basisszenario, vorausgesetzt, dass die HG-SIT die Angst vor den Folgen anaphylaktischer Stichreaktionen nimmt.

Fazit: Die geschätzten Kosten pro QALY einer HG-SIT in Deutschland sind als kosteneffektiv anzusehen, da sie unter gängigen Schwellenwerten (NICE: 30.000 £ pro QALY) liegen.

\section{P6.6}

Verlauf der antigenspezifischen T-Zellantwort während der Wespengift-Immuntherapie

\section{S. Saleh', P. Bacher ${ }^{2}$, G. Heine', A. Scheffold ${ }^{3}$, M. Worm ${ }^{1}$}

${ }^{1}$ Allergologie und Immunologie, Klinik für Dermatologie, Venerologie und Allergologie, Charité - Universitätsmedizin Berlin, Berlin, Deutschland

${ }^{2}$ Abteilung für zelluläre Immunologie, Klinik für Rheumatologie and klinische Immunologie, Charité Universitätsmedizin Berlin, Berlin, Deutschland ${ }^{3}$ Deutsches Rheuma Forschungszentrum (DRFZ) Berlin, Leibniz-Gemeinschaft, Berlin, Deutschland
Die spezifische Immuntherapie (SIT) ist eine effektive Therapie. Dennoch sind die immunologischen Mechanismen insbesondere die Veränderungen antigenspezifischer T-Zellen nicht vollständig verstanden und Studien durch geringe T-Zellfrequenzen in der Peripherie begrenzt. In der vorliegenden Untersuchung wurden antigenspezifische T-Zellantwort während einer Wespengift-Immuntherapie charakterisiert. Hierfür wurde ein neuartiges antigenspezifisches T-Zell-Anreicherungs-Protokoll (ARTE: antigen-specifc T-cell enrichment) eingesetzt, das es ermöglichen geringe Mengen antigenspezifischer T-Zellen durchflusszytometrisch zu untersuchen.

Die antigenspezifische T-Zellantwort von 20 Wespengiftallergikern wurde vor SIT sowie 1, 3 und 7 Wochen nach der Einleitungsphase mit ARTE charakterisiert. Nach Stimulation wurden Wespengift-spezifische T-Zellen via CD40L (CD154) magnetisch angereichert und analysiert (CD4+, CD45RO+, CD154+, IL-2+, IL-4+, IL-10+, IFN- $\gamma+$ ). Weitere 6 Probanden wurden auf IL-5+, IL-9+, IL-13+ und Ki-67+ T-Zellen vor SIT und eine Woche nach der Einleitungsphase analysiert.

Es trat ein signifikanter Anstieg von CD154+ CD4+ T-Zellen und CD154+ IL4+ T-Zellen zu Beginn der SIT auf, auf den ein schneller Rückgang zu Ausgangswerten folgte. Ähnliche Ergebnisse erhielten wir bei der Analyse von IL-5, IL-13, Ki-67, IL-2 und CD45-RO produzierenden T-Zellen. Ein Anstieg von IFN- $\gamma+$ und IL10 + T-Zellen war hingegen zu keinem Zeitpunkt detektierbar.

Unsere Daten zeigen, dass wiederholte Wespengiftinjektionen nach einem initialen Anstieg zu einer Abnahme IL-4, IL-5 und IL-13 produzierender TH2-Zellen führt, jedoch zu keinem Anstieg antigenspezifischer IFN- $\gamma+$ und IL10+ T-Zellen. Schlussfolgerung: Die Verwendung des neuartigen ARTE-Protokolls ermöglicht die Untersuchung antigenspezifischer T-Zellen direkt und ex-vivo, wodurch Artefakte durch zeitaufwendige ex-vivo Methoden vermieden werden.

\section{P6.7}

\section{Verträglichkeit der Umstellung einer Einleitungs- Rush Insektenimmuntherapie mit lyophilisierten Allergenextrakten zu einem Depotpräparat}

\section{R. Scarpone, M. Kraft, M. Worm}

Allergologie und Immunologie, Klinik für Dermatologie, Venerologie und Allergologie, Charité - Universitätsmedizin Berlin, Berlin, Deutschland

Die spezifische Immuntherapie (SIT) mit Insektengift wird in der Regel stationär mit wässrigen Allergenextrakten eingeleitet und ambulant mit an Aluminiumhydroxid adsorbierten Depotextrakten weitergeführt.

In dieser retrospektiven Analyse wurde die Verträglichkeit der Umstellung von einem lyophilisierten Extrakt zu einem Depotpräparat untersucht.

Es wurden 220 Patientenakten (2003-2018) ausgewertet. 200 Patienten wurden mit Wespengift, 20 mit Bienengift therapiert. Hiervon waren 128 (58\%) weiblich und 92 (42\%) männlich, mit einem Durchschnittsalter zu Beginn der Therapie von $51( \pm 14)$ Jahren. Der Schweregrad der anaphylaktischen Insektenstichreaktion nach Müller betrug bei 42 (19\%) Patienten Grad 2, bei 101 (46\%) Grad 3 und bei 77 (35\%) Grad 4, mit durchschnittlich gIgE 206,32 $( \pm 31,75) \mathrm{kU} / \mathrm{l}$, sIgE Wespe $11,16( \pm 1,16) \mathrm{kU} / \mathrm{l}$, sIgE Biene $3,78( \pm 0,87) \mathrm{kU} / \mathrm{l}$ und basaler Serumtryptasekonzentration $6,42( \pm 0,48) \mu \mathrm{g} / \mathrm{l}$.

Aufgetretene systemische Nebenwirkungen während der SIT wurden gemäß der WAO Gradeinteilung klassifiziert. Während 
der Therapieeinleitung hatten 8 (4\%) Patienten mit Wespengift SIT eine Reaktion, davon 6 (3\%) Grad 1 und 2 (1\%) Grad 2. Nach der ersten Gabe des Depotpräparates hatten $3(1.5 \%)$ Patienten eine Reaktion, 2 (1\%) Grad 1 und 1 (0.5\%) Grad 2.

Bei 3 (15\%) Patienten mit Bienengift SIT trat in der Einleitungsphase eine Reaktion auf, bei $2(10 \%)$ Grad 1 und bei 1 (5\%) Grad 2. Bei der Umstellung auf das Depotpräparat traten keine Reaktionen auf.

Die Daten zeigen, dass die Verträglichkeit einer Umstellung von lyophilisiertem Insektengift während der Einleitungsbehandlung zu einem Depotpräparat sehr gut ist und unkompliziert durchgeführt werden kann.

\section{P6.8}

Tiotropium add-on therapy improves lung function in children and adolescents with symptomatic moderate and severe asthma, independent of markers of allergic status

\section{Vogelberg', E. Hamelmann ${ }^{2,3}$, G. El Azzi ${ }^{4}$, M. Engel ${ }^{4}$, R. Sigmund ${ }^{5}$, S. Szefler ${ }^{6}$}

${ }^{1}$ Department of Pediatric Pulmonology and Allergy, University Hospital Carl Gustav Carus, Technical University of Dresden, Dresden, Germany

${ }^{2}$ Klinikum für Kinder- und Jugendmedizin, Evangelisches Klinikum Bethel, Bielefeld, Germany

${ }^{3}$ Allergy Center, Ruhr University, Bochum, Germany

${ }^{4} \mathrm{TA}$ Respiratory Diseases, Boehringer Ingelheim International $\mathrm{GmbH}$, Ingelheim am Rhein, Germany

${ }^{5}$ Biostatistics and Data Sciences, Boehringer Ingelheim Pharma GmbH \& Co. KG, Biberach an der Riss, Germany ${ }^{6}$ Department of Pediatrics, Children's Hospital Colorado, University of Colorado Denver School of Medicine, Aurora, Colorado, USA

Objectives: Assess whether allergic status impacts lung function improvements with tiotropium (tio) add-on therapy in 6-17-year-old patients with symptomatic moderate and severe asthma despite inhaled corticosteroid (ICS) treatment \pm other controllers.

Method: Analyses involved four Phase III, randomised, double-blind, placebo(pla)-controlled, parallel-group trials in patients aged 6-11 years and 12-17 years (severe symptomatic asthma; NCT01634152/NCT01277523, moderate symptomatic asthma; NCT01634139/NCT01257230). Patients received once-daily tio or plavia Respimat ${ }^{\circledR}$ as add-on to ICS \pm other controllers. Post hoc modelling analyses included change from baseline (response) in peak forced expiratory volume in 1 second (FEV1) within 3 hours post-dose (FEVl(0-3 h); primary endpoint in each study), trough (predose) FEV1 response, FEV1/forced vital capacity (FVC) ratio, forced expiratory flow (FEF25-75\%) response and in-clinic trough (evening) peak expiratory flow (PEF) response in pooled datasets at the time of primary endpoint assessment (severe asthma: Week 12; moderate asthma: week 24) across the full range of baseline blood eosinophil counts $(0-2000$ cells $/ \mu \mathrm{L})$ and total serum IgE levels $(0-2000 \mu \mathrm{g} / \mathrm{L})$.

Results: 1590 patients were treated. Significant improvements were observed with tio versus pla in peak FEVl $(0-3 \mathrm{~h})$ response, trough FEV1 response, FEV1/FVC ratio, FEF25-75\% response, and in-clinic trough (evening) PEF response. These improvements were independent of the magnitude of baseline IgE levels and blood eosinophil counts.
Conclusion: Once-daily tio as an add-on to ICS maintenance therapy \pm other controllers improves lung function in children and adolescents with moderate and severe symptomatic asthma, irrespective of IgE levels and blood eosinophil counts.

\section{P6.9}

\section{Effect of tiotropium Respimat $₫$ on seasonal asthma worsening in paediatric patients}

\section{Vogelberg', S. Szefler ${ }^{2,3}$, S. Goldstein ${ }^{4}$, L. Graham ${ }^{5}$, G. El Azzi ${ }^{6}$, M. Engel ${ }^{6}$, L. Zaremba-Pechmann ${ }^{7}$, E. Hamelmann 8,9}

${ }^{1}$ Klinik und Poliklinik für Kinder- und Jugendmedizin, Universitätsklinikum Carl Gustav Carus, Technische Universität Dresden, Germany

${ }^{2}$ The Breathing Institute, Children's Hospital of Colorado, Aurora, Colorado, USA

${ }^{3}$ Department of Pediatrics, School of Medicine, University of Colorado, Aurora, Colorado, USA

${ }^{4}$ Allergy and Asthma Care of Long Island, Rockville Centre, New York, USA

${ }^{5}$ Pediatric Pulmonology, Children's Healthcare of Atlanta, Atlanta, GA, USA

${ }^{6}$ TA Respiratory Diseases, Boehringer Ingelheim Pharma $\mathrm{GmbH} \& \mathrm{Co}$. KG, Ingelheim am Rhein, Germany ${ }^{7}$ Biostatistics, Boehringer Ingelheim Pharma GmbH \& Co. KG, Biberach an der Riss, Germany

${ }^{8}$ Klinik für Kinder und Jugendmedizin, Evangelisches Klinikum Bethel, Bielefeld, Germany

${ }^{9}$ Allergy Center, Ruhr University, Bochum, Germany

Background: We aimed to investigate the adverse events (AEs) related to asthma exacerbations and asthma symptoms that were reported across several paediatric trials investigating the safety and efficacy of tiotropium (tio). Of particular interest was the identification of any seasonality pattern in the reporting of these AEs, whilst also ascertaining whether tio efficacy was consistent throughout the year.

Methods: We pooled data from 5 randomised, doubleblinded, placebo(pla)-controlled trials investigating the safety and efficacy of tio in paediatric asthma: NinoTinA-asthma ${ }^{\circ}$ (NCT01634113); CanoTinA-asthma (NCT01634139); VivaTinAasthma ${ }^{\circledR}$ (NCT01634152); RubaTinA-asthma ${ }^{\circledR}$ (NCT01257230); PensieTinA-asthma ${ }^{\circ}$ (NCT01277523). In all trials, patients received once daily tio $(5 \mu \mathrm{g}$ or $2.5 \mu \mathrm{g})$ or pla via Respimat ${ }^{\ominus}$, in addition to at least inhaled corticosteroids. AEs related to asthma exacerbations or symptoms were defined using a composite endpoint "asthma exacerbations and asthma-related symptoms". The number of asthma exacerbations reported as AEs were plotted by month, with data from the Southern hemisphere shifted by 6 months to align the seasons.

Results: Overall 1,691 patients aged 1-17 years were included in the pooled analyses. The rate of patients reporting AEs related to asthma exacerbations and symptoms, number of patients with an event per 100 patients-years, was reduced with tio $5 \mu \mathrm{g}$ compared with pla (rate ratio $[\mathrm{RR}] 0.76$ [95\% confidence interval (CI) $0.63,0.93]$ ), with a similar trend associated with tio $2.5 \mu \mathrm{g}$ (RR 0.87 [95\% CI 0.72, 1.05]).

When analysed by month, reports of AEs related to asthma exacerbations and symptoms were greatest in the pla group in the spring, autumn and winter, and lowest in summer. With both doses of tio, spring and autumn peaks were reduced. 
Conclusion: This analysis suggests treatment with tio reduces AEs related to asthma exacerbations and symptoms in paediatric patients, with particular effect in reducing spring and autumn seasonal peaks were reduced.

\section{P6.10}

Type 2 biomarkers associated with dupilumab efficacy in patients with uncontrolled, moderateto-severe asthma enrolled in the phase 3 study Liberty Asthma Quest

\section{S. E. Wenzel', I. Pavord' ${ }^{2}$, H. Zhu ${ }^{3}$, B. Zhang ${ }^{3}$, J. Maroni ${ }^{4}$, P. Rowe ${ }^{3}$, J. Hamilton ${ }^{4}$, B. Swanson ${ }^{3}$, N. Amin', G. Pirozzi ${ }^{3}$, N. Graham ${ }^{4}$, A. Teper ${ }^{3}$}

${ }^{1}$ Asthma Institute, University of Pittsburgh, Pittsburgh, PA, USA

${ }^{2}$ Respiratory Medicine Unit, University of Oxford, Oxford, UK ${ }^{3}$ Sanofi, Bridgewater, NJ, USA

${ }^{4}$ Regeneron Pharmaceuticals, Inc., Tarrytown, NY, USA

Rationale: Dupilumab, a fully human VelocImmune ${ }^{\oplus}$ derived anti-IL-4R $\alpha \mathrm{mAb}$ that inhibits signaling of IL-4 and IL-13, key drivers of Type 2 inflammation, is approved for treatment of adults with inadequately controlled moderateto-severe atopic dermatitis (AD). In a phase 3 asthma study (NCT02414854), dupilumab 200/300 mg every 2 weeks vs matched placebo reduced annualized severe exacerbation rates (AER) during the 52-week treatment period and improved pre-bronchodilator (BD) forced expiratory volume in 1 second (FEV1), \% change in FEV1, asthma symptoms, and quality of life measures, and was generally well tolerated in the intent-to-treat population. This post hoc study evaluates associations between baseline (BL) Type 2 biomarkers and therapeutic responses to dupilumab.

Method: Treatment-by-BL Type 2 biomarker interactions were determined using negative-binomial regression spline models for AER over 52 weeks and regression spline models for pre-BD FEV1 at Week 12.

Results: For AER: treatment-by-BL biomarker interactions observed for eosinophils $(P<0.0001)$ and fractional exhaled nitric oxide (FeNO; $P<0.0001)$ with both dupilumab regimens. For change from BL in pre-BD FEV1 at Week 12: interactions observed for eosinophils $(P<0.0001)$, FeNO $(P<0.0001)$, periostin $(P=0.0009)$, total IgE $(P=0.0245)$, and eotaxin-3 $(P=0.0151)$ for both regimens. The most frequent adverse event in dupilumab-treated groups vs placebo was injection-site reactions $(15 \% / 18 \%$ vs $5 \% / 10 \%$, respectively). In contrast to dupilumab studies in $\mathrm{AD}$, conjunctivitis rates were similar for dupilumab and placebo.

Conclusions: Baseline Type 2 biomarkers including eosinophils, FeNO, periostin, total IgE, and eotaxin-3 showed treatment-by-BL biomarker interactions for FEVl; only eosinophils and FeNO showed interactions for AER. This suggests that differences in biologic pathways for lung function and exacerbations exist, but eosinophils and FeNO are common to both and may be better biomarkers for treatment decisions.

\section{P6.11}

\section{Adjuvante Omalizumab-Gabe erleichtert die Venom-Immuntherapie bei Risikopatienten}

\section{J. Wobser ${ }^{1}$, F. Prenzel ${ }^{2}$, K. Berndt', J. Simon', R. Treudler}

${ }^{1}$ Klinik und Poliklinik für Dermatologie, Venerologie und Allergologie, Universitätsklinikum Leipzig, Deutschland ${ }^{2}$ Klinik und Poliklinik für Kinder- und Jugendmedizin, Universitätsklinikum Leipzig, Leipzig, Deutschland ${ }^{3}$ Klinik und Poliklinik für Dermatologie, Universitätsklinikum Carl Gustav Carus, Dresden, Deutschland

Hintergrund: Unerwünschte Soforttyp-Reaktionen können die Einleitung einer Venom-Immuntherapie (VIT) erschweren. Es existieren kasuistische Berichte über die verbesserte Verträglichkeit der VIT nach Gabe einer adjuvanten Therapie mit dem Anti-IgE-Antikörper Omalizumab bei Risikopatienten. Wir berichten über eine entsprechende Fallserie aus Sachsen.

Methodik: Betrachtet wurden $n=6$ Patienten (2F, 4M; 11-76 Jahre, 2 Bienen- und 4 Wespengiftallergiker) aus insgesamt 3 Zentren mit $\geq 1$ VIT-Abbruch infolge einer anaphylaktischen Reaktion $\geq$ II. Grades nach Ring/Messmer. Dokumentiert wurden spezifisches IgE gegen das allergieauslösende Insekt, Gesamt-IgE, Dosis, Intervall und Dauer der Omalizumab-Gabe sowie die Verträglichkeit bei neuerlicher VIT-Einleitung.

Ergebnisse: Medianes Gesamt-IgE: 315,5 kU/1 (46,61164,0); Wespengiftallergiker: medianes IgE Wespengift: 3,76 $\mathrm{kU} / 1(2,1-26,4)$, Ves v $1: 1,56 \mathrm{kU} / 1(0,62-3,56)$, Ves v 5: 1,06 kU/1 (0,50-29,30); Bienengiftallergiker: medianes IgE Api m 1: 2,32 kU/1 (0,41-55,50); mediane Serum-Tryptase: 32,35 $\mu \mathrm{g} / \mathrm{l}$ $(3,3-300,0)$; systemische Mastozytose bei $n=3$. Omalizumab (300 mg, s.c.)-Injektionen erfolgten bei $n=4 \mathrm{im}$ Vorfeld der VIT (je 3 Injektions-Zeitpunkte, Intervall: 9 Wochen-1 Tag vor VIT), bei $n=2$ einmalig während VIT-Einleitungsphase. Fortsetzungsbehandlung: Omalizumab bei $n=4$ begleitend zur VIT über 4-95 Wochen, Intervall: 2-6-wöchentlich; bei $n=2$ fortlaufend, Intervall 4-6 Wochen, aktuell im 6. Jahr. Alle Patienten zeigten eine gute Verträglichkeit der VIT nach bzw. unter Omalizumabgabe.

Diskussion: Adjuvante Omalizumabinjektionen ermöglichten die VIT bei Patienten mit stattgehabter schwerer anaphylaktischer Reaktion während vormaliger VIT. Bislang fehlen einheitliche Empfehlungen zu Injektionsintervall und -dauer.

\section{Drug allergy and skin diseases}

\section{P7.1}

\section{IgE-mediated reaction to chlorhexidine: an unexpected cause of intraoperative anaphylaxis}

\section{T. Bangerl, W. Hötzenecker, I. Angelova-Fischer}

Klinik für Dermatologie, Kepler Universitätsklinikum, Linz, Austria

Chlorhexidine is widely used as an antiseptic and disinfectant. Chlorhexidine coatings, introduced into medical devices such as urinary catheters or impregnated central venous catheters 
or chlorhexidine-based topical agents are important yet often unrecognised cause of perioperative anaphylaxis.

A 64-year old patient was referred to our department because of intraoperative anaphylaxis during colon surgery and suspicion of rocuronium bromide hypersensitivity. Within a few minutes after the start of the operation the patient experienced generalised flush and urticaria. The performed skin prick tests with neuromuscular blocking agents, opioids, hypnotics, soy, latex, cephalosporines, penicilline and non-steroidal anti-inflammatory agents were negative. In contrast, the prick tests with chlorhexidine $5 \mathrm{mg} / \mathrm{ml}$ and $5 \mathrm{mg} / \mathrm{ml}$ in $1: 10$ dilution were positive. Furthermore, the performed in vitro diagnostik showed the presence of specific IgE to chlorhexidine (27.6 $\mathrm{kU} / \mathrm{l})$. Studying of the anaesthesia chart in detail revealed that the patient received a urinary catheter immediately before the operation pointing that chlorhexidine, contained in the gel of the urinary catheter, was the likely cause of the reaction.

Allergic reactions to chlorhexidine have been associated with delayed type of sensitization however immediate type of reactions, including urticaria, angioedema or anaphylactic shock after chlorhexidine exposure are increasingly being described in the literature. Even topical application has been reported to elicit systemic anaphylactic reactions and the patients may undergo several episodes of unexplained anaphylaxis until diagnosed. Based on the literature and as shown in our case, even if not mentioned in the anaesthesia protocol, chlorhexidine exposure and allergy must be considered a possible cause of perioperative anaphylaxis and the patients should undergo detailed allergology testing to prevent further anaphylactic reactions.

\section{P7.2}

Pilotstudie zur Untersuchung der Wirksamkeit und Verträglichkeit einer Basistherapie begleitend zur topischen Kortikosteroid-Therapie bei Kindern

\section{Cappello', E. González Salteiro²}

${ }^{1}$ LETI Pharma GmbH, Ismaning, Deutschland 'Laboratorios LETI S. L. U., Barcelona, Spanien

Einleitung: Atopische Dermatitis (AD) ist eine chronisch entzündliche Erkrankung, die durch eine defekte Barrierefunktion der Haut charakterisiert ist, insbesondere in ekzematösen Arealen. Die Basistherapie der AD unterstützt die Reparatur der Hautbarriere und soll auch begleitend zur medikamentösen Therapie angewendet werden.

Ziel: Untersuchung der Wirksamkeit und Verträglichkeit einer begleitendenden Intensivpflege als Basistherapie, im Rahmen einer topischen Kortikoid-Therapie bei Kindern mit AD.

Methode: 30 Kinder (2-12 Jahre) mit vergleichbaren Ekzemen auf beiden Körperhälften wurden in einer randomisierten, einfach verblindeten Studie eingeschlossen. In einem Split-Body-Design wurden die Ekzeme entweder nur mit topischen Kortikoiden (Kl. 3, Kontrolle) oder mit topischen Kortikoiden (Kl. 3) plus Basistherapie mit LETIAT4 Intensivcreme (Behandlungsarm) über 28 Tage behandelt. Die Kortikoide wurden $1 \times$ täglich bis zur Remission und die Basistherapie mind. $2 \times$ täglich aufgetragen. Die Wirksamkeit und Verträglichkeit wurde mittels lokalem SCORAD sowie einer allgemeinen Beurteilung durch den Dermatologen und den Probanden ermittelt.

Ergebnisse: Es wurde eine gegenüber der Kontrolle signifikante Besserung des lokalen SCORAD um durchschnittlich $16 \%$ erzielt. Eine Verbesserung des lokalen SCORAD zeigte sich bei $90 \%$ der Probanden in der Behandlungsgruppe, gegenüber $79 \%$ in der Kontrollgruppe. Im Behandlungsarm zeigte sich nach 14 Tagen eine signifikant bessere Juckreizlinderung (25\%) gegenüber der Kontrolle. Eine signifikante Verkürzung der Kortikoidanwendung (Behandlungsarm vs. Kontrolle: 6,9 vs. 9,4 Tage) konnte bei $24 \%$ der Kinder beobachtet werden. Die Basistherapie wurde sehr gut vertragen.

Zusammenfassung: In dieser Pilotstudie konnte gezeigt werden, dass eine begleitende tägliche Basistherapie mit LETIAT4 Intensivcreme zu einer signifikant besseren Linderung der Symptome führte als bei alleiniger Kortikoidtherapie, bei sehr guter Verträglichkeit.

\section{P7.3}

Psoriasis reduziert das Auftreten von spezifischen Kontaktallergien

\section{A. Claßen 1 , T. Buhl', S. Schubert ${ }^{2}$, M. Margitta Worm ${ }^{3}$,} A. Bauer ${ }^{4}$, J. Geier ${ }^{2}$, S. Molin ${ }^{5}$

${ }^{1}$ Abteilung Dermatologie, Venerologie und Allergologie, Universitätsmedizin Göttingen, Göttingen, Deutschland ${ }^{2}$ Informationsverbund Dermatologischer Kliniken (IVDK), Universität Göttingen, Göttingen, Deutschland ${ }^{3}$ Klinik für Dermatologie, Allergologie und Venerologie, Allergie-Centrum-Charité, Charité - Universitätsmedizin Berlin, Berlin, Deutschland

${ }^{4}$ Klinik für Dermatologie, Universitätsklinikum Carl Gustav Carus, Technische Universität Dresden, Dresden, Deutschland

${ }^{5}$ Klinik für Dermatologie und Allergologie, Ludwig Maximilians Universität, München, Deutschland

Hintergrund: Bisher durchgeführte Studien beschreiben eine inverse Assoziation zwischen Kontaktallergie und Psoriasis, wobei die Ergebnisse der bisher publizierten Daten widersprüchlich sind. Untersuchungen zur Genexpression in Patienten mit einer Kontaktallergie zeigen, dass verschiedene Haptene auch unterschiedliche Immunantworten induzieren. Während Nickel eine Thl/Th17-dominierte Immunantwort auslöst, sind Duftstoffe für eine überwiegend Th2-gerichtete Immunantwort verantwortlich.

Ziel der Untersuchung: Es sollte überprüft werden, ob in einer großen multinationalen Kohorte eine Assoziation zwischen Kontaktallergien und Psoriasis besteht, und ob Psoriatiker ein spezifisches epikutanes Sensibilisierungsprofil aufweisen. Patienten und Methoden: Retrospektive Auswertung von Daten des Informationsverbundes Dermatologischer Kliniken (IVDK) aus den Jahren 1996-2015. In dieser Zeit wurden 2387 Psoriatiker und 161.989 Kontrollpatienten epikutan getestet.

Ergebnisse: Eine Kontaktallergie fand sich bei Psoriatikern signifikant seltener $(\mathrm{OR}=0,545)$. Die logistische Regressionsanalyse mit mehreren unabhängigen Variablen zeigt einen „protektiven Effekt“ der Psoriasis für die meisten Allergene, unabhängig vom Alter, Geschlecht, betroffenen Körperpartie und Indikation zur Epikutantestung. Der Duftstoffmix II $(\mathrm{OR}=0,36)$ und Wollwachsalkohole $(\mathrm{OR}=0,38)$ zeigten die geringsten Sensibilisierungsraten, wohingegen Nickelsulfat nur minimal geringere epikutane Sensibilisierungsraten im Vergleich zu Kontrollpatienten aufwies $(\mathrm{OR}=0,75)$.

Schlussfolgerung: Der inverse Zusammenhang von Kontaktallergien und der Systemerkrankung Psoriasis ist möglicherweise nicht nur durch eine generell verringerte Reaktionsbereitschaft des Individuums erklärbar. Liegt bereits eine 
Polarisation des Immunsystems bei einer immunologischvermittelten Systemerkrankung vor, könnte das Auftreten von spezifischen Kontaktallergien je nach immunologischem Reaktionsmuster beeinflusst werden.

\section{P7.4}

\section{Die Langzeit-Rhinoflowmetrie zur Beurteilung der Nasenatmung}

\section{A. Darbinjan, T. Hummel, M. Cuevas}

HNO-Klinik, Universitätsklinikum Dresden, Dresden, Deutschland

Einleitung: Die operative Septumkorrektur gehört zu den häufigsten HNO-Eingriffen. Zur Beurteilung des OP-Erfolgs standen bisher neben den subjektiven Angaben durch den Patienten die anteriore Rhinoskopie und die Rhinoresistometrie zur Verfügung. Diese Methoden sind jedoch nur Momentaufnahmen ohne Berücksichtigung eines längeren Zeitintervalls, beeinflusst zum Beispiel durch den Nasenzyklus. Die Rhinoflowmetrie zeichnet über 24 Stunden seitengetrennt den nasalen Luftfluss, die Herz- und Atemfrequenz auf und liefert objektive Angaben über die Durchlässigkeit der Nase und somit das Operationsergebnis.

Methoden: Wir untersuchten 20 Patienten vor und 3 Monaten nach Nasenseptumplastik. Neben verschiedenen Fragebögen (SNOT-20, Fragebogen zur Bedeutung der Geruchswahrnehmung) wurde der HNO-ärztliche Spiegelbefund erhoben und eine chronische Sinusitis ausgeschlossen. Zur funktionellen Beurteilung erfolgte ein Riechtest mit Sniffin' Sticks, die Rhinoresisto- und akustische Rhinometrie. Sowohl ein paar Tage vor der Operation als auch 3 Monate nach dem Eingriff wurde die $24 \mathrm{~h}$-Rhinoflowmetrie durchgeführt. Die gleichen Untersuchungen wurden an einer Kontrollgruppe ohne Operation durchgeführt.

Ergebnisse: Es zeigt sich, dass es zu einer Verbesserung des nasalen Flows nach der Operation kommt. Dieses Ergebnis korreliert mit den Fragebögen und den anderen erhobenen Befunden. Bei der Kontrollgruppe gab es keine signifikanten Änderungen.

Schlussfolgerung: Die Rhinoflowmetrie ist ein gutes methodisches Mittel, um den Therapieerfolg nach Septumplastik zu objektivieren. Wir planen, die Rhinoflowmetrie auch zur Diagnostik von vor allem ganzjährigen Allergien bzw. zur objektiven Verlaufskontrolle unter spezifischer Immuntherapie einzusetzen.

\section{P7.5}

\section{Antihistaminika-induzierte Urtikaria}

\section{B. Kreft, A. Gebert, S. Jahnke, C. Sunderkötter}

Klinik und Poliklinik für Dermatologie und Venerologie, Universitätsklinikum Halle (Saale), Martin-Luther-Universität, Halle-Wittenberg, Halle (Saale), Deutschland

Histamin-1-Rezeptor-Antagonisten der 2. Generation finden unter anderem Verwendung zur Symptomkontrolle bei der allergischen Rhinitis oder der Urtikaria. Sie zeichnen sich aus durch eine außerordentlich gute Verträglichkeit bei fehlenden oder kaum sedierenden Effekten. Das Auftreten urtikarieller
Hautveränderungen nach Einnahme von Antihistaminika ist in der Literatur kasuistisch wiederholt beschrieben.

Wir berichten über eine 33-jährige Patientin, bei der jeweils nach Einnahme verschiedener Antihistaminika, aber auch nichtsteroidaler Antirheumatika (NSAID), kleine stecknadelkopfgroße Urtikae betont an den Unterarmen auftraten. Durchgeführte Hauttestungen waren negativ, so dass eine immunologisch-bedingte Typ-I-Allergie nicht sehr wahrscheinlich erschien. Die Analyse der Einzelinhaltsstoffe der jeweils provozierten Antihistaminika ergab keine übereinstimmenden Hinweise für eine Unverträglichkeit auf die Hilfsstoffe. Somit wurde ursächlich eine Intoleranzreaktion auf Antihistaminika und NSAID angenommen.

Der Pathomechanismus Antihistaminika-induzierter urtikarieller Hautveränderungen ist bisher nicht eindeutig verstanden. Diskutiert werden nichtimmunologische Intoleranzphänomene durch eine unspezifische Mastzelldegranulation, eine Fehlfunktion von H1-Rezeptoren, abnorme Metabolisierungswege oder Mechanismen der Komplementaktivierung. Andererseits sind in Einzelfällen auch Typ-I-allergische Reaktionen beschrieben. Ursächlich für Unverträglichkeiten auf mehrere Antihistaminika dürften Kreuzreaktivitäten zwischen den unterschiedlichen Gruppen der Piperidine und Piperazine aufgrund struktureller Ähnlichkeiten sein. Ob es einen kausalen pathophysiologischen Zusammenhang zur Analgetika-Intoleranz gibt, ist unklar. Das kombinierte Auftreten einer Unverträglichkeit auf Antihistaminika und einer Analgetika-Intoleranz, wurde aber unlängst beschrieben.

\section{P7.10}

\section{Anaphylaxie und Chemotherapie: \\ Eine Auswertung von Daten aus dem Anaphylaxie-Register}

\section{G. Poch1', S. Dölle', K. Scherer², M. Worm ${ }^{1}$}

${ }^{1}$ Abteilung Allergologie und Immunologie, Klinik für Dermatologie, Venerologie und Allergologie, Charité Universitätsmedizin Berlin, Berlin, Deutschland ${ }^{2}$ Allergie Abteilung, Klinik für Dermatologie, Universitätsklinikum, Basel, Schweiz

Anaphylaxien können schwere und lebensbedrohliche Ereignisse in der Allergologie darstellen. Medikamente gehören neben Insektengiften und Nahrungsmitteln zu den drei häufigsten Auslösern von Anaphylaxien.

Ziel der der vorliegenden Untersuchung ist die Häufigkeit und Risikofaktoren von Anaphylaxien gegenüber Chemotherapeutika mit Hilfe der Daten aus dem Anaphylaxie-Register zu analysieren.

Hierzu wurden die Daten des Anaphylaxie-Registers bzgl. Auslösern, Symptomen, Risikofaktoren, Medikation, Komorbidität und Therapie von anaphylaktischen Reaktionen nach der Gabe von Chemotherapeutika zwischen 2007-18 ausgewertet.

11596 registrierte Fälle wurden untersucht. $19 \%(n=2150)$ der Fälle waren mit der Gabe von Medikamenten assoziiert. Innerhalb dieser Gruppe beträgt der Anteil von Anaphylaxien durch Chemotherapeutika $2 \%(n=37) .62 \%$ dieser Reaktionen trat bei Platinderivaten, $27 \%$ bei Taxanen, $8 \%$ bei Alkylanzien und $3 \%$ bei Vincaalkaloiden auf. Frauen waren häufiger betroffen $(84 \%$ vs. $16 \%, p<0,01)$. Andere Faktoren wie Alter, Gewicht, pulmonale Vorerkrankungen oder die Menopause waren unabhängig. Die Schwere der Anaphylaxie war nicht mit bestimmten Chemotherapeutika assoziiert. Patienten mit einer allergischen 
Rhinokonjunktivitis reagieren häufiger auf platinhaltige Chemotherapeutika als auf Taxane ( $23 \%$ vs. $10 \%, p=0,03$ ).

Anaphylaxien auf Chemotherapeutika sind selten. In Übereinstimmung mit der aktuellen Literatur treten allergische Reaktionen am häufigsten gegenüber Platin- oder Taxan-Chemotherapeutika auf. Risikofaktoren wie Gewicht, respiratorische Erkrankungen und Menopause konnten in dieser Kohorte nicht bestätigt werden. Jedoch konnten wir ein erhöhtes Risiko für Anaphylaxien gegenüber Platinderivaten bei Patienten mit allergischer Rhinokonjunktivitis identifizieren. Größere Fallzahlen sind notwendig um das Risiko von Anaphylaxien gegenüber Chemotherapeutika zu identifizieren um Patienten präventiv zu schützen.

\section{P7.11}

\section{Vom Antidepressivum zur Polyserositis - Eine seltene Differenzialdiagnose}

\section{K. Stamos ${ }^{1}$, G. Hahn ${ }^{2}$, H. Taut ${ }^{3}$, C. Vogelberg ${ }^{4}$}

${ }^{1}$ Fachbereich päd. Pneumologie/Allergologie, Unikinderklinik Dresden, Dresden, Deutschland

${ }^{2}$ Kinderradiologie, Dresden, Deutschland

${ }^{3}$ Pädiatrische Sonographie, Klinik und Poliklinik für Kinderund Jugendmedizin, Universitätsklinikum Carl Gustav Carus, Dresden, Deutschland

${ }^{4}$ Fachbereich pädiatrische Pneumologie/Allergologie,

Klinik und Poliklinik für Kinder- und Jugendmedizin, Universitätsklinikum Carl Gustav Carus, Dresden, Deutschland

Anamnese: Initiale Aufnahme eines 17-jährigen Mädchens mit akutem Abdomen und V.a Appendizitis bei fieberhaften Temperaturen, rechtsseitigen Unterbauchschmerzen und Arthralgien. Nach Appendektomie progrediente Dyspnoe, anhaltendes Fieber, beginnende Polyserositis, Pleuraergüsse bds und verminderte Belastbarkeit.

Dauermedikation: Fluoxetin seit 08/2017

Entlassung nach $20 \mathrm{~d}$ in die Häuslichkeit bei Fieberfreiheit mit in Reduktion befindlichem systemischen Kortikosteroid. Wiederaufnahme nach Absetzen des Kortikosteroids bei AZVerschlechterung und erneutem Fieber.

Befunde und Verlauf: Status: reduzierter AZ; Lid- und Fußrückenödeme, RaRi gerötet mit Stippchen, Pulmo: abgeschwächtes AG re>li, hyposonorer KS, cervikale LKS bds, inguinale gerötete strangförmige derbe Struktur, druckdolent, sonstiger Status unauffällig.

Rö-Thx: Pleuropneumonie bds, progrediente Ergüsse und Dystelektasen bds. Sonographie Pleura: Pleuraergüsse ca. $7 \mathrm{~cm}$ bds, im Verlauf Progredienz mit massiven Binnenechos und pneumonischen Infiltrationen. Unter Drainagenanlage deutliche Regredienz.

Abd. Sonographie: Massiver Aszites mit Binnenechos retrovesikal, verwaschene Mark-Rinden-Diefferenzierung Nieren bds, Pankreas mit inhomogener Textur und Echogenität.

Echokardiographie: Geringer Perikarderguß, keine Endokarditis.

Labor: max. CrP $260 \mathrm{mg} / \mathrm{l}$, Leukozytose, LDH/Leberwerte/ proBNP/IL2 erhöht, Blutkultur/Pleurapunktat/Aszites unauffällig.

Klinischer Verlauf: Absetzen des Fluoxetins. Unter Prednisolon massive Leukoplakie und Cushing Syndrom, so dass nach 1 Monat systemische Kortikosteroide über 1 Monat langsames Ausschleichen. Darunter Regredienz der Symptomatik.
Schlussfolgerung: Bei einer Konstellation von langanhaltendem Fieber, Lymphknotenschwellung, Eosinophilie, Exanthem sowie weiterer Organbeteiligung mit Besserung nur unter systemischen Kortikosteroiden ohne jeglichen Erregernachweis oder Hinweis einer autoinflammatorischen Erkrankung, sollte an ein DRESS gedacht werden.

\section{P7.12}

\section{Assessment of quality of life in patients} with chronic urticaria

\section{Tavakkol}

Alborz University of Medical Sciences, Karaj, Iran

Chronic urticaria (CU) is one of the most common chronic skin disorders. This condition has significant impact on the patients' quality of life (QoL). Assessment of QOL is one of the most useful guides for the physicians to evaluate the severity of the disease and the effect of therapy. Disease specific questionnaires are necessary for this purpose. The single validated disease specific questionnaire, initially created in Italian language and was translated to several languages, is Chronic Urticaria Quality of Life Questionnaire (CU-Q2oL). We designed this survey to develop the Persian version of the CU-Q2oL. CU-Q2oL was translated into Persian on the basis of standard guidelines. A group of 110 patients were diagnosed by an allergist to suffer from CU on the basis of guidelines were enrolled. Patients were asked to fill out the validated Persian version of questionnaire. The severity of the CU was assessed by using urticaria activity score 7 (UAS7). The Exploratory Factor Analysis was done on the 6 dimensional scale of Persian CU-Q2oL. Approximately $68 \%$ of the variance by these 6 factor structure was found to be higher than $59.9 \%$ of the original Italian version. The internal consistency, measured by Cronbach Alfa factor, for all 6 factors was shown to be appropriate. The QOL was significantly affected by the severity of CU, measured by UAS7, UAS7 score and coincidence of angioedema predicted th CU-Q2oL score. We found that Persian version of CU-Q2oL could be used as a valid and reliable way for the clinical and research purposes. 